\title{
FEASIBILITY STUDY OF A RAILGUN AS A DRIVER FOR IMPACT FUSION
}

Final Report

Y. C. Thio

Principal Investigator

V. Dellarchi, J. Dugan, L. S. Frost, W. Mamrose, D. L. Ometz, F. Stefani, V. V. Subramaniam, P. L. Ulerich, T. M. Valko, H. R. Wilkinson, D. M. York, J. M. Zomp Investigators

Westinghouse R\&D Center

1310 Beulah Road

Pittsburgh, PA 15235

June 1986

\author{
Prepared for \\ R. Gajewski \\ Technical Program Manager
}

\author{
U.S. Department of Energy \\ under Contract No. DE-ACO2-83ER13048
}

\section{DISCLAIMER}

This report was prepared as an account of work sponsored by an agency of the United States Government. Neither the United States Government nor any agency thereof, nor any of their employees, makes any warranty, express or implied, or assumes any legal liability or responsibility for the accuracy, completeness, or usefulness of any information, apparatus, product, or process disclosed, or represents that its use would not infringe privately owned rights. Reference herein to any specific commercial product, process, or service by trade name, trademark, manufacturer, or otherwise does not necessarily constitute or imply its endorsement, recommendation, or favoring by the United States Government or any agency thereof. The views and opinions of authors expressed herein do not necessarily state or reflect those of the United States Government or any agency thereof. 


\section{DISCLAIMER}

This report was prepared as an account of work sponsored by an agency of the United States Government. Neither the United States Government nor any agency Thereof, nor any of their employees, makes any warranty, express or implied, or assumes any legal liability or responsibility for the accuracy, completeness, or usefulness of any information, apparatus, product, or process disclosed, or represents that its use would not infringe privately owned rights. Reference herein to any specific commercial product, process, or service by trade name, trademark, manufacturer, or otherwise does not necessarily constitute or imply its endorsement, recommendation, or favoring by the United States Government or any agency thereof. The views and opinions of authors expressed herein do not necessarily state or reflect those of the United States Government or any agency thereof. 


\section{DISCLAIMER}

Portions of this document may be illegible in electronic image products. Images are produced from the best available original document. 


\section{Table of Contents}

ABSTRACT $\ldots \ldots \ldots \ldots \ldots \ldots \ldots \ldots \ldots \ldots \ldots \ldots \ldots \ldots \ldots \ldots \ldots \ldots \ldots \ldots$

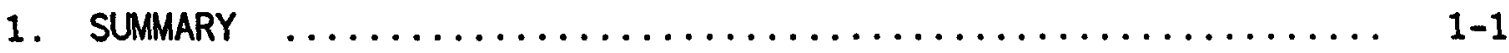

2. ABLATION AND VISCOUS DRAG: THEIR EFFECTS ON LAUNCH DYNAMICS.. 2-1

2.1 Effect of Ablation on Arc Dynamics with no Viscous Drag . 2-2

2.2 The Effect of Viscous Drag without Arc Mass Accumulation 2-5

2.3 Combined Effects of Ablation and Viscous Drag ......... 2-8

2.4 Discussion, Results, and Conclusion................ 2-11

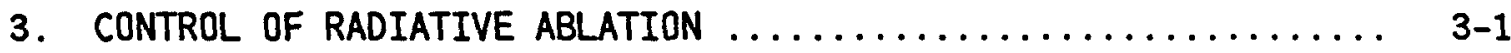

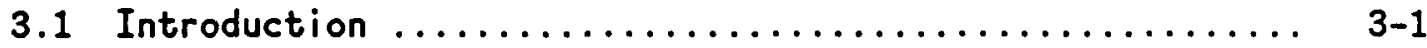

3.2 The Physical Framework and the Method of Analysis ...... 3-3

3.3 Armature Resistance, Temperature, and Length ......... 3-4

3.4 Plasma Radiative Heating of the Wall Materials......... 3-13

3.5 Magnetic (or Joule) Heating of the Rails ............ 3-15

3.6 Minimum Armature Velocity for Wall Survivability ....... 3-16

3.7 Conclusion ................................. 3-20

4. ARMATURE STABILITY OR INSTABILITY.................. 4-1

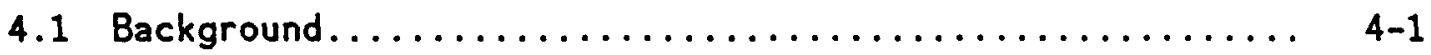

4.2 Derivation of the Perturbation Equations............. 4-2

4.3 Solving the Perturbed Equations................... 4-6

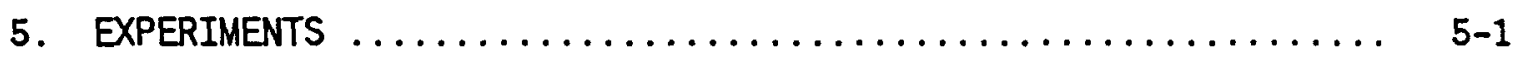

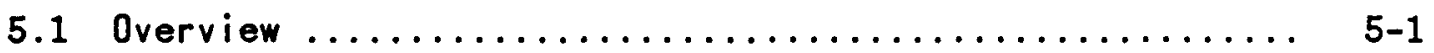

5.2 Experimental Results Summary $\ldots \ldots \ldots \ldots \ldots \ldots \ldots \ldots \ldots, 5-1$

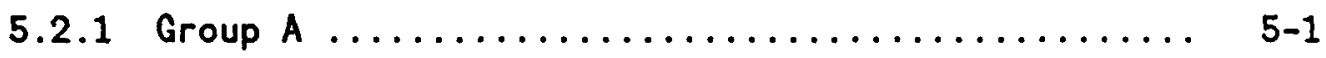

5.2 .2 Group $B \ldots \ldots \ldots \ldots \ldots \ldots \ldots \ldots \ldots \ldots \ldots \ldots \ldots, 5-8$



5.2 .4 Group $D \ldots \ldots \ldots \ldots \ldots \ldots \ldots \ldots \ldots \ldots \ldots \ldots \ldots, 5-10$

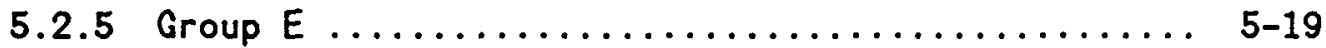

5.2 .6 Group $F \ldots \ldots \ldots \ldots \ldots \ldots \ldots \ldots \ldots \ldots \ldots \ldots, 5-22$

5.3 Remarks $\ldots \ldots \ldots \ldots \ldots \ldots \ldots \ldots \ldots \ldots \ldots \ldots \ldots \ldots \ldots \ldots, \quad 5-35$ 
6. MECHANICALLY CONTROLLED PLASMA ARMATURES $\ldots \ldots \ldots \ldots \ldots \ldots \ldots .6-1$

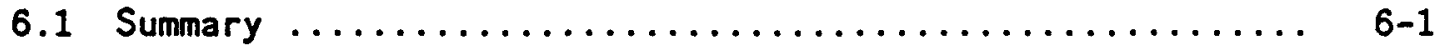

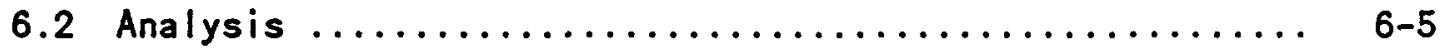

6.2 .1 Overview ......................... 6-5

6.2.2 Model for the Plasma in the Arc Chamber ....... 6-6

6.2.3 Adiabatic Flow through the Nozzle ............ 6-11

6.2.4 Flow in the Diverging Section with Heat Loss .... 6-15

6.3 Feasibility: A Conceptual, Point Design Analysis ...... 6-17

6.4 Launch Dynamics of Mechanically Controlled Plasma

Armature ............................... 6-22

6.5 Advanced Augmentation Geometry and Trans-Augmentation ... 6-31

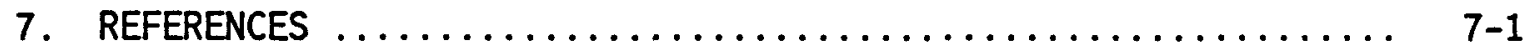




\section{Abstract}

The feasibility of a railgun as a driver for impact fusion is studied through a series of theoretical and experimental investigations. The results of both the theoretical and experimental investigations presented here have helped to identify the potential problems of the railgun launcher to attain velocity in excess of $100 \mathrm{~km} / \mathrm{s}$. These include ablation, viscous drag, and secondary arc formation due to either armature dispersion (instability) or restrike. These problems are analysed and examined experimentally. The behavior of the conventional open-plasma-armature driven railguns have been shown to be quite complex and not easily controllable in the domain of ultrahigh velocity ( $>6 \mathrm{~km} / \mathrm{s})$. Methods to overcome these problems are proposed, analysed in regards to their technological feasibility, and tested experimentally wherever possible. Techniques for reducing radiative ablation, the concept of a mechanically controlled plasma armature, and the concept of achieving super high augmentation by the technique of trans-augmentation are presented. We conclude that, with the use of the mechanically controlled plasma armature coupled with a technique for achieving high augmentation, it is quite feasible for railguns to produce velocities in excess of $50 \mathrm{~km} / \mathrm{s}$. However, much further research and development of the underlying physical and technological foundations to support the development of such ultrahigh velocity launchers are required. 


\section{Summary}

The feasibility of a railgun as a driver for impact fusion is studied through a series of theoretical and experimental investigations. The results of both the theoretical and experimental investigations presented here have identified the potential problems of the railgun launcher to attain velocity in excess of $100 \mathrm{~km} / \mathrm{s}$. These include ablation, viscous drag, and secondary arcs formation due to either armature dispersion (instability) or restrike.

To overcome radiative ablation by the open plasma armature, we have been driven to consider the use of cold, low atomic weight plasma armatures. While this was verified experimentally to produce significantly less ablation, the plasma armature tends to be long and unstable. While for low velocity applications (below $20 \mathrm{~km} / \mathrm{s}$ ), the length and its tendency to be unstable are not limiting factors, these two aspects become extremely important for application above $50 \mathrm{~km} / \mathrm{s}$. A greater length of the armature gives rise to greater viscous drag on the arc, while the lack of stability is a pre-disposition for the formation of secondary arcs. A third factor is the condition of the gaseous products in the bore immediately after the passage of the fast moving armature. For very high velocity application, the bore is required to withstand a high voltage immediately behind the armature.

To overcome these problems, the concept of a 'plasma clipper' is developed, in the form of a slotted projectile. A study of the scientific and technological feasibility is made. The results show that, not only that it should be technologically feasible, the technique has the potential capability of suppressing the formation of secondary 
arcs, and controlling the arc mass hence the viscous drag, and to produce a gaseous environment in the bore which is capable of withstanding the high back emf produced by the moving armature. It appears quite possible to use the slotted projectile railgun technique to achieve velocity in excess of $50 \mathrm{~km} / \mathrm{s}$ and producing velocity required for impact fusion with further research. 


\section{Ablation and Viscous Drag: Their Effects on Launch Dynamics}

The earlier experiments of several railgun research groups, including those of Hawke et. al.[1], Thio et. al.[2], Bedford et. al.[3], Clark and Bedford[4], and Bedford[5], indicated that severe ablation of wall materials is commonplace in railguns in which the arc was generated by exploding metallic foil of relatively low mass. Before the initiation of the present program, mass removal from the barrel materials were typically of the order of a gram per shot in railguns whose bore are no more than 1 square centimeter and using projectiles of only 1 or 2 gram. For the ultimate application, where barrel life of the order of $10^{4}$ to $10^{6}$ shots are expected, this level of erosion rate is clearly unacceptable.

Recognizing the need to eliminate or greatly reduce this ablation, we undertook to investigate the feasibility of an engineering solution to the problem right from the outset of this program in 1983. This is complemented by the companion program sponsored by the U.S. Army Research Office $[6,7,8]$. Apart from the question of barrel life, the ablated mass tends to be carried along by the arc, and absorbs some of the momemtum of the arc, giving rise to reduced acceleration (an inertial drag effect). It was Parker et. al. [9], who first clearly identified and announced the possibility of severe degradation in the acceleration of the plasma armature if the materials ablated from the wall were completely entrained in the arc. Parker et. al. also gave a first estimate of the effects of viscous drag on the launch dynamics. However, in Parker et. al., the theory was not extensively used or applied to cover a sufficiently broad range of system, plasma, and material parameters which are relevant to our study here in the context of impact fusion. 
In this chapter, we present our analysis of the effects of ablation and viscous drag on the performance of a single-shot launcher and its implications in relation to the goal of impact fusion. The analysis applies and extends Parker's theory in several directions, and in a few critical aspects differs from it. Firstly, we assume that the arc resistance (including resistance of the rail-arc boundary layer) has only a very weak dependence on the arc current, giving rise to an increasing arc voltage with increasing current instead of a constant arc voltage. Secondly, we allow for the possibility of partial instead of full ablation of the wall materials by the arc radiant energy. In particular, we allow for the possibility of eventually el iminating or greatly reducing the ablation of the thermally conductive rails.

Thirdly, we also allow for the possibility of incomplete entrainment of the ablated materials in the arc. The difference these assumptions make to the implications of the theory is shown to be rather significant. The extended theory is then applied to obtain results relevant to the problem of impact fusion.

Section 2.1 analyses the implication of ablation alone without viscous drag. In Section 2.2 we analyse the implication of viscous drag without mass accumulation in the arc. This case has not been analysed before. In Section 2.3 an analysis of the combined effects of ablation and viscous drag is given. In Section 2.4 a discussion of the results of the theory is given.

\subsection{EFFECT OF ABLATION ON ARC DYNAMICS WITH NO VISCOUS DRAG}

The railgun equation of motion allowing for a changing arc mass and no viscous drag is,

$$
\frac{d v}{d t}=\left(\frac{1}{2} L^{\prime} I^{2}-\dot{m}_{A} v\right) /\left(m_{A}+m_{p}\right)
$$


where $m_{p}$ is the projectile mass, $m_{A}$ is the instantaneous mass of the plasma armature, $L$ ' is the inductance gradient, $I$ is the current and $v$ is the velocity. The increase of arc mass due to ablation appears as the term involving $\dot{m}_{A}$. We see that the effect of ablation is to produce a reduction in the acceleration of the arc and projectile (an inertial drag). This acceleration degradation is proportional to velocity. For a constant current, the arc will undergo an acceleration until a critical velocity $\theta$, is reached, when the acceleration will be reduced to zero. The critical velocity $\theta$, is given by equating the R.H.S. of Equation (2.1) to zero:

$$
\theta_{1}=\frac{L^{\prime} I^{2}}{2 \dot{m}_{A}}
$$

The value of this critical velocity depends on the value of the time rate of increase of the arc mass. Parker et. al.[9], postulates that all the radiated energy from the plasma armature goes into ablating the wall materials. Since the radiated energy is equal to the ohmic dissipation in the arc, the postulate leads to an expression for $m_{A}$ in the form,

$$
\dot{m}_{A}=\alpha I V_{A}
$$

where $a$ is a material constant specifying the amount of ablated mass per unit of energy absorbed by the material, $V_{A}$ is the voltage drop across the arc which includes both the electrode drop and the voltage drop across the arc column. Substituting (2.3) into (2.2), we obtain the critical velocity in terms of the ablation coefficient $a$ which is characteristic of the wall materials, and the total arc voltage $V_{A}$,

$$
\theta_{1}=\frac{L^{\prime} I}{2 a V_{A}}
$$

The above is Parker's expression for the critical velocity. 
We can generalize the above expression as follows. Let us assume that the radiation from the plasma armature is isotropic. The total radiant energy received by the rails and the insulators separately is then proportional to the surface area exposed to the arc of each of these walls. Suppose only a fraction of the radiant energy succeeds in ablating the rails or the insulator. And suppose not all the ablated mass is entrained with the arc. Then the rate of increase of the arc mass is

$$
\dot{m}_{A}=\left(\frac{\beta_{a, 1} s_{r} a_{a, 1}+\beta_{a, 2^{s}} \alpha_{a, 2}}{s_{r}+s_{i}}\right) I V_{A}
$$

where $a_{a, 1}$ and $a_{a, 2}$ are the ablation coefficients of the rail and insulator materials respectively, $s_{r}$ is the height (or circumferential distance) of the exposed part of the rail, $s_{i}$ is the width (or circumferential) of the exposed part of the insulator, and the $\beta$ 's are coefficients which we introduce here to account for the partial ablation or entrainment of the wall materials. The $\beta$ 's have values between 0 and 1. We call them the active ablation fractions. Finally, by expressing the arc voltage in terms of an equivalent arc resistance $R_{A}$, we can rewrite the critical velocity in a form not involving the driving current explicitly as,

$$
\theta_{1}=\left(\frac{s_{r}+s_{i}}{\beta_{a, 1} s^{a} a, 1+\beta_{a, 2} s_{i}^{a} a, 2}\right) \frac{L^{\prime}}{2 R_{A}}
$$

We can define an effective ablation coefficient $a_{b}$ as,

$$
\alpha_{b}=\frac{\beta_{a, 1} s_{r}^{\alpha} a, 1^{+} \beta_{a, 2} s_{a, 2}^{\alpha}}{s_{r}+s_{i}}
$$

and re-write Expression (2.6) for the critical velocity in the form,

$$
\theta_{1}=\frac{L^{\prime}}{2 a_{b} R_{A}}
$$


An estimate for the arc resistance $R_{A}$ from past experiments with small-bore $(1 \mathrm{~cm})$ railgun experiments may be taken to be approximately 1 $\mathrm{m}$. Consider a $1-\mathrm{cm}$ square-bore railgun in which tungsten rails and lexan insulator are used as wall materials for the bore in equal proportions. Assuming that the active ablation fractions have the value of unity, the effective ablation coefficient $a_{b}$ for this gun is $48 \times 10^{-9} \mathrm{~kg} / \mathrm{J}$. A simple square-bore railgun (without augmentation), has typically an inductance gradient of about $0.3 \mu \mathrm{H} / \mathrm{m}$. This gun would have a critical velocity of the order of $3 \mathrm{~km} / \mathrm{s}$. This would be an absolute limiting velocity for this gun if all the radiation energy succeeds in ablating the wall materials, and if all the ablated materials get entrained in the plasma armature. If only the insulator materials is ablated, the effective ablation coefficient $a_{b}$ would be $5 \times$ $10^{-9} \mathrm{~kg} / \mathrm{J}$. The limiting (critical) velocity in that case is of the order of $35 \mathrm{~km} / \mathrm{s}$, a substantial increase over the case when both the tungsten and the insulator is ablated. Further if the arc resistance could be halved, the critical velocity would be raised to $70 \mathrm{~km} / \mathrm{s}$. Finally, if an augmented railgun is used in which the effective inductance gradient $L^{\prime}$ is $3 \mu \mathrm{H} / \mathrm{m}$, the critical velocity would be over $700 \mathrm{~km} / \mathrm{s}$.

This simple calculation illustrates very clearly the sensitivity of the critical velocity $\theta_{1}$ to the 3 critical parameters: the effective ablation coefficient, the arc resistance, and the effective inductance gradient of the railgun.

\subsection{THE EFFECT OF VISCOUS DRAG WITHOUT ARC MASS ACCUMULATION}

While the reduction of acceleration due to ablation is proportional to velocity, that due to viscous drag on the plasma armature is proportional to velocity squared. At sufficiently high velocity, viscous drag dominates over ablation as a mechanism in limiting the velocity attainable by a given railgun. The equation of motion for the arc and projectile which includes the effect of viscous drag but assuming no cummulative increase in arc mass is given by, 


$$
\left(m_{p}+m_{a}\right) \frac{d v}{d t}=\frac{1}{2} L^{\prime} I^{2}-\frac{1}{2} c_{f} m_{A} v^{2}\left(I_{b} / A_{b}\right)
$$

where $c_{f}$ is the skin friction coefficient between the plasma armature and the wall, $I_{b}$ and $A_{b}$ are the perimeter and the crossectional area of the bore respectively. By equating its R.H.S. to zero, the draglimited velocity can be obtained from (2.9) as,

$$
v_{f}=\left(\frac{L^{\prime} I^{2} A_{b}}{c_{f} m_{A} I_{b}}\right)^{1 / 2}
$$

We may express this in term of the peak gas pressure $p_{b}$ to be contained in the bore,

$$
v_{f}=\left(\frac{2 p_{b} A_{b}^{2}}{c_{f} m_{a} l_{b}}\right)^{1 / 2}
$$

Given a fixed maximum bore pressure, bore crossectional area and perimeter, and a fixed value for the skin friction coefficient $c_{f}$, one is left with the arc mass as the only control paramter which may be varied in order to achieve a given velocity. This critical arc mass for a given velocity is given by,

$$
m_{A}=\frac{2 p_{b} A_{b}^{2}}{c_{f} v^{2} I_{b}}
$$

The main difficulty of using any of the above expressions is the uncertainty in estimating the value for the skin friction coefficient, $c_{f}$. A lot more experimental and theoretical investigations are required here. The limited experiments conducted on this program (see later) provide estimates for $c_{f}$ in the range of 0.001 to 0.003 . Parker 
et. al., [9] also give experimental estimates for $c_{f}$ in the same range. Tidman and Goldstein[10], using a turbulent boundary layer theory of Schlicting[11] for incompressible flow, give an estimate for the skin friction coefficient as,

$$
c_{f}=\left\{\frac{1}{2\left[2 \log _{10}(a / \delta a)+1.74\right]} \quad \frac{\bar{u}}{u}\right\}^{2}, c_{f}=\frac{\lambda}{4}\left(\frac{\bar{u}}{u}\right)^{2}
$$

where $\bar{u}$ is the mean velocity of the flow and $u$ is the axial velocity, is obtained from the solution of the following transcendental equation,

$$
\frac{1}{\sqrt{\lambda}}=1.74-2 \log _{10}\left[\frac{\delta a_{w}}{a}+\frac{18.7}{R_{e} \sqrt{\lambda}}\right]
$$

in which a is the bore diameter, $\delta a_{w}$ is the wall roughness, and $R_{e}$ is the Reynold number. For a turbulent flow, $\bar{u} / u$ is typically about 0.86 . By developing expressions appropriate for a partially ionized gas, Tidman and Goldstein estimate that in typical operation of railguns, the Reynold number for the plasma armatures is typically in the range of $10^{6}$ to $10^{8}$. Practical values for wall roughness is rarely better than 1 $\mu \mathrm{m}$. With these values, Tidman-Goldstein expression (2.13) gives an estimate of 0.002 for the skin coefficient. Calculations using a turbulent boundary layer code for compressible flow for a perfectly smooth wall, however, have predicted values for the skin friction an order of magnitude lower[12].

Given a maximum bore pressure of $300 \mathrm{MPa}$, a bore crossectional area of $1 \mathrm{~cm}^{2}$, a bore perimeter of $4 \mathrm{~cm}$, and a skin friction coefficient $c_{f}$ of 0.001 , in order to obtain a drag-limiting velocity above $100 \mathrm{~km} / \mathrm{s}$, the maximum allowable arc mass can be calculated from (2.12) to be $15 \mathrm{mg}$. If the skin friction coefficient could be reduced to 0.0001 , 
then the arc mass can be as much as $150 \mathrm{mg}$ before the viscous drag would limit the velocity to below $100 \mathrm{~km} / \mathrm{s}$.

\subsection{COMBINED EFFECTS OF ABLATION AND VISCOUS DRAG}

When both ablation and viscous drag are present, the nature of the interplay between these two effects depend on the actual values of the effective ablation rate $a_{b}$ and the skin friction coefficient. When the effective ablation rate is high and the skin friction coefficient low, an ablation limiting velocity according to Expression (2.8) could be reached before viscous drag has any significant effect on the dynamics of the arc. Conversely, if the ablation rate is low and the skin friction coefficient high, then viscous drag will dominate in limiting the velocity of the accelerator. The complication introduced by ablation in the latter case is that the arc mass is constantly changing. If there is no mass reduction mechanism from the arc, the mass of the arc will monotonically increase with time thereby increasing the viscous drag. In the case of a constant current drive, when the arc has accumulated enough mass to produce an instantaneous viscous drag and ablation drag equalling the Lorentz force, the arc would stop accelerating. Beyond that point, the ablated mass would increase the viscous drag beyond the applied Lorentz force produced by the constant current, consequently the arc would deccelerate.

Following Parker et. al., [9] we can calculate this viscous-dragdominated limiting velocity $v_{m}$ in a self-consistent way by starting with the equation of motion taking account of both the inertial drag caused by ablation and the viscous drag as follows,

$$
\left(m_{P}+m_{A}\right) \frac{d v}{d t}=\frac{1}{2} L^{\prime} I^{2}-\frac{1}{2} c_{f} m_{A} v^{2} I_{b} / A_{b}-\dot{m}_{A} v
$$

A discussion of the solution of this equation in the case of a constant current and arc voltage has been given by Parker et. al., to 
which the reader is referred for further details. We shall present here only a discussion of the application and the implication of the solution of the equation.

The maximum velocity attainable by the railgun whose performance conforms to Equation (2.14) can be obtained by setting the R.H.S. of (2.14) to zero. Using Parker's notation $v_{m}$ for the maximum velocity, we obtain $v_{m}$ as the solution to the quadratic

$$
\frac{c_{f} \theta_{1} t_{m}}{2 I_{H}}\left(\frac{v_{m}}{\theta_{1}}\right)^{2}+\frac{v_{m}}{\theta_{1}}-1=0, \quad I_{H}=\frac{A_{b}}{I_{b}}
$$

where $t_{m}$ is the time at which the maximum velocity is reached. Writing

$$
\tau_{m}=\frac{c_{f} \theta_{1} t_{m}}{2 I_{H}}
$$

we may write the solution of $(2.15)$ as,

$$
\frac{v_{m}}{\theta_{1}}=\frac{-1+\left(1+4 \tau_{m}\right)^{1 / 2}}{2 \tau_{m}}
$$

From integrating the differential Equation (2.14) in a dimensionless form numerically, Parker et al found that the time to reach maximum velocity can be approximated with an accuracy of a few percent by,

$$
\tau_{\mathrm{m}}=1.817 \beta^{3 / 5}
$$

in which the parameter $\beta$ is given by Parker et. al., in the form,

$$
\beta=\frac{c_{f} m_{p} L^{\prime}}{D\left(a V_{a}\right)^{2}}
$$


Further, by assuming the parameter $\beta$ to be large, Parker et. al., gives an approximation to the maximum velocity in the form,

$$
v_{m}=0.371 \frac{\left(L^{\prime}\right)^{0.7} D^{0.3} I}{\left(a V_{a}\right)^{0.4}\left(c_{f} m_{p}\right)^{0.3}}
$$

We shall not use the approximate form (2.20) here. For the purpose of the analysis here where the parameter $\beta$ may vary over a wide range we shall use the more exact form, Expression (2.17), recast in the following form,

$$
v_{m}=\left[\frac{-1+\left(1+7.268 \beta^{3 / 5}\right)^{1 / 2}}{3.634 \beta^{3 / 5}}\right] \theta_{1}
$$

Further, we shall not use Parker's form for the parameter $\beta$. Instead we shall express $\beta$ as,

$$
\beta=2 \theta_{1}^{2} \theta_{2}
$$

where $\theta_{2}$ is defined by,

$$
\theta_{2}=\frac{c_{f}}{4 a I_{h}}
$$

where a is a "nominal acceleration" defined in terms of the maximum bore pressure or the applied Lorentz force by,

$$
a=\frac{\frac{1}{2} L^{\prime} I^{2}}{m_{p}}=\frac{P_{b} A_{B}}{m_{p}}
$$

With reference to Equation (2.21), we see that the maximum velocity $v_{m}$ is determined in terms of the two critical para-design parameters, $\theta_{1}$ and $\theta_{2}$. 


\subsection{DISCUSSION, RESULTS, AND CONCLUSION}

In the context of impact fusion, most of the projectile can be made to consist essentially of a light-weight sabot. In this case, we may further reduce the parameter $\theta_{2}$ to a form closer to engineering as follows,

$$
\theta_{2}=c_{f}\left(\frac{P_{p}}{P_{b}}\right)\left(\frac{L}{D}\right)
$$

where $\rho_{p}$ is the density of the sabot, $L / D$ is the aspect ratio of the sabot and $P_{b}$ is the maximum bore pressure. The choice of the working bore pressure is determined by the mechanical and material engineering of the barrel. In terms of Expression (2.8) and (2.25), the two parameters $\theta_{1}$ and $\theta_{2}$ have been given in a form which does not involve the driving current nor the bore diameter explicitly. Implicitly, however, the parameter $\theta_{1}$ depends on the driving current and the bore diameter but only very weakly through the arc resistance. It could, however, depend strongly on the driving current through the effective ablation coefficient $a_{b}$. In general, the effective ablation coefficient will decrease rapidly with decreasing bore current density (see later). Thus, in general, a lower bore current density would give a larger value for $\theta_{1}$ and a correspondingly larger value for the limiting velocity $v_{m}$, all other things being equal.

Based upon the present state of the art in the mechanical and material cngineering of the barrel, it is not unreasonable to assume a value of $250 \mathrm{MPa}$ for the working bore pressure. A futuristic value for this may be up to 4 times higher. Over the last decade, polycarbonate (Lexan) has been found by various experimenters time and again to be the sabot of choice. Its density is approximately $1200 \mathrm{~kg} / \mathrm{m}^{3}$. Though we expect to see mechanically stronger and thermally more resistant sabot material in the future, significant reduction of the sabot density below that of polycarbonate is unlikely. Assuming for now a bore pressure of 
$250 \mathrm{MPa}$ and a sabot density of $1000 \mathrm{~kg} / \mathrm{m}^{3}$, a practical expression for the parameter $\theta_{2}$ can be given as,

$$
\theta_{2}=4 \times 10^{-6} c_{f} \quad s^{2} m^{-2}
$$

For values of the skin friction coefficient $c_{f}$ ranging from $5 x$ $10^{-3}$ to $5 \times 10^{-5}, \theta_{2}$ would vary from $2 \times 10^{-8}$ to $2 \times 10^{-10}$.

In Figure 2.1 we show the maximum velocity $v_{m}$ as 1 imited by ablation and viscous drag only (see later for additional performance losses) versus the parameter $\theta_{1}$. Each curve corresponds to a fixed value of $\theta_{2}$. The ensemble shown in Figure 2.1 represents a variation in $\theta_{2}$ and hence the skin friction coefficient $c_{f}$ over 2 orders of magnitude. Similarly, the results have also been presented for values of $\theta_{1}$ varying over 2 orders of magnitude from $10 \mathrm{~km} / \mathrm{s}$ to $1000 \mathrm{~km} / \mathrm{s}$.

Taking the results for $\theta_{2}=10^{-9}$ as representative, it can be seen from Figure 2.1 that, in order to achieve velocity in the range of impact fusion, very high values are required for $\theta_{1}$. Specifically, to achieve velocity above $50 \mathrm{~km} / \mathrm{s}, \theta_{1}$ would need to be at least $500 \mathrm{~km} / \mathrm{s}$. This requires exceedingly high value for the effective inductance gradient L' $(2-4 \mu \mathrm{H} / \mathrm{m})$ for the accelerator, an exceedingly low value for the effective ablation coefficient $\alpha_{b}(2-5 \mu \mathrm{g} / \mathrm{J})$ and the arc resistance $R_{A}$ (below $0.5 \mathrm{mn}$ ).

The development of concepts and their experimental testings to verify their scientific feasibility to eventually realize the impact fusion domain of these three critical parameters, $L^{\prime}, a_{b}$ and $R_{A}$ has been one of the main concerns and thrusts of the investigations on this program. We proceed to describe this effort in the next few chapters. 


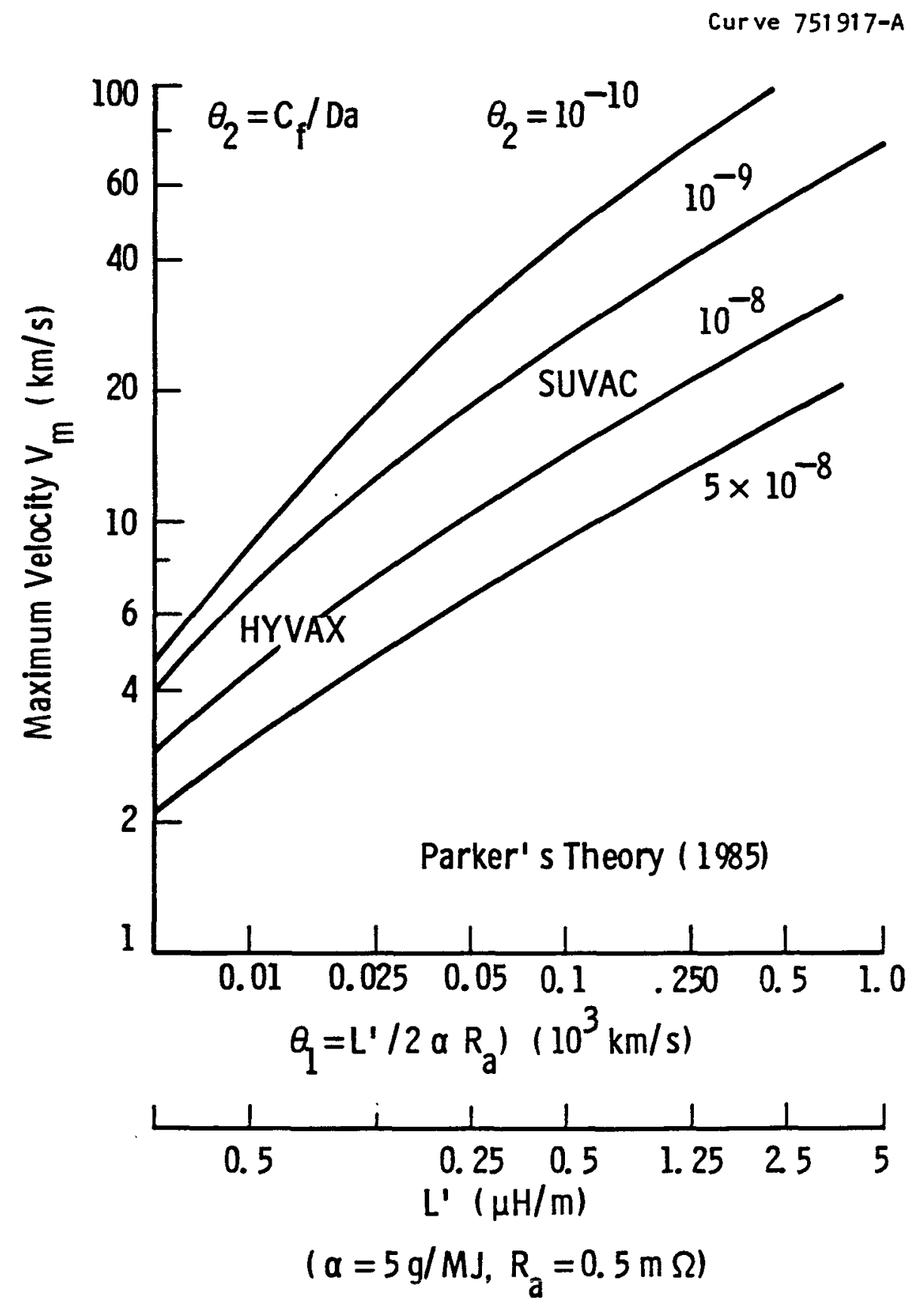

Figure 2.1 - The combined effects of ablation and viscous drag on maximum velocity attainable in railguns with constant current device. 


\section{Control of Radiative Ablation}

\subsection{INTRODUCTION}

In Chapter 2, we see the importance of being able to el iminate or at least reduce the effective ablation coefficient and the arc resistance. In this chapter, we describe the results of our investigations which are conducted with the aim of examining the feasibility of achieving this dual objective - reducing ablation and arc resistance.

Wall ablation in railguns is a result of many complex processes occuring at the rail-armature and rail-insulator interface. From classical arc studies, the presence of cathode spots and sometimes anode spots are well known[13-17]. In these arc roots, extremely high concentrations of current occur. In many conventional applications of electric arcs in which the mean current density is much smaller than that experienced in railguns, radiation intensity from the arc column (arc interior) is relatively low and insignificant. Under those conditions, the energetic processes in the arc roots dominate the erosion of the electrodes, leading to erosion rates typically in the 1 - $100 \mu \mathrm{g}$ per coulomb of charge transfer.

Unfortunately, this does not appear to be the situation in the case of railgun arcs. Firstly, because of the much higher current density, the mean radiation intensity from the arc body is relatively high. Secondly, the typical erosion rates measured in mass per unit charge transfer observed in railguns is in the range of 10 $30 \mathrm{mg} / \mathrm{C},[18]$ approximately 3 orders of magnitude above those observed in conventional arcs. 
The first step in our study to examine the feasibility of controlling the ablation problem is to identify the principal mechanism(s) which is (are) responsible for this great difference in the erosion rate between railgun arcs and more conventional arcs. We pose a simple but fundamental question: Could the mean thermal flux from the main body of the arc in railguns be sufficient to cause melting, vaporization and hence ablation of the wall materials? If so, what are the key parameters governing this mean thermal flux? Is there a domain of suitable properties and structures for the plasma armature so that ablation caused by the mean thermal flux of the arc could be eliminated?

The present work represents one of a continuing series of efforts to address these issues in a systematic way. It is a significant extension of an earlier study by Powell[19] in that we explore the answers to these questions in a much larger parameter space. It is also an extension of an earlier work by Thio[7] in that non-ideal plasma effects are included in the present study.

The dominant contributions to the mean heat flux from the arc are radiation, turbulent thermal conduction and energy generated by hypervelocity skin friction at the wall. It must be noted that the turbulent thermal conduction and skin friction heat generation can be as large or greater than the radiative transfer under certain circumstances [20]. Marshall[21,22] has recently also drawn attention to the presence of arc roots in railgun arcs. The result of these additional heat transfer mechanisms is to aggravate the wall ablation problem in railguns. An excellent and independent study, which carefully treats the various forms of energy and momentum transfer to the wall from the arc through the plasm-wall boundary layer, has just been recently completed by Tidman, Goldstein and Winsor[20]. In this study, we have focused our attention on the radiative contribution. 
The study of the radiative behavior of the plasma armature requires the use of a model of the plasma armature. In so doing, we are automatically led to studying the nature of the dependence of the resistance of the main body of the arc on the system and armature parameters. Thus we are able to study the feasibility and the methods of reducing the arc resistance in a companion fashion to the ablation problem. In this study we assume that the resistance of the armaturerail boundary layer is small (approximately 10\%) compared to the main body of the arc.

\subsection{THE PHYSICAL FRAMEMORK AND THE METHOD OF ANALYSIS}

Consider a stable plasma armature moving at constant acceleration and endowed with a uniform current density, a configuration of minimum peak current concentration for a given total current and spatial extent of the plasma armature. For a given set of gun parameters (such' as bore size and rail inductance gradient) and plasma parameters (such as its total mass and ionic species), using the results developed in Thio[6,7] for ideal railgun plasmas or Thio and Frost [8] for non-ideal railgun plasma arcs, we can calculate the temperature and the length of the plasma armature. The radiative flux from the arc on to the rails and insulators is then determined and this in turn determines the rate of temperature rise in the materials. To this we add the magnetic heating of the rails caused by the pulsed current flowing in them. The length of the plasma armature determines its dwell time at a given position of the wall as it flies by. Together, the radiative heat flux, the rate of thermal energy generation by magnetic heating and the dwell time determine whether the melting point of the material would be exceeded during the fly-by of the plasma armature.

In the work reported in Year 1 (1983) of the present program[23], we used the ideal railgun plasma armature theory of Thio[7]. We have since extended our plasma armature model to include non-ideal plasma effects. In this chapter, we report on the more recent 
results obtained from applying the railgun arcs theory of Thio and Frost which includes non-ideal plasma effects. By appropriately treating the non-ideal plasma behavior, the modelling results should have a higher degree of approximation to the practical situations.

\subsection{ARMATURE RESISTANCE, TEMPERATURE, AND LENGTH}

Using the model of Thio and Frost[24], we made calculations for an exemplary case of a railgun which has a $1-\mathrm{cm}$ square bore and an inductance gradient $L^{\prime}$ of $0.32 \mu \mathrm{H} / \mathrm{m}$. The calculations are made for currents between $200 \mathrm{kA}$ and $400 \mathrm{kA}$ and unity has been assumed for all emissivities. The Thio-Frost scaling factor $s_{T}$ has been assigned the value of 0.5 .

Figure $3.1,3.2$ and 3.3 shows respectively the estimated resistance, temperature and voltage of the arc column versus the total number of particles (ions and neutral atoms) in the armature. When the number of particles is small, say $5 \times 10^{20}$, the armature tends to be short and the temperature and the resistance tend to be high.

In the case of a pure Li plasma, the resistance is of the order of $1 \mathrm{~m} n$ and is relatively insensitive to variation in current. The temperature is relatively high: approximately $25,000 \mathrm{~K}$ for a current of $200 \mathrm{kA}$ and $45,000 \mathrm{~K}$ at $400 \mathrm{kA}$. The armature length is relatively short. At these temperatures, the lithium plasma is essentially ideal $(\gamma / \bar{z}<<$ 0.1 ). Thus, the electron-ion contribution of the resistivity here could have been well approximated by Spitzer's expression. Further, due to the relatively low ionization potential of lithium, the Li plasma is nearly fully ionized (Figures 3.4 and 3.5 ).

Thus the contribution to the resistivity from the electronneutral scattering is small due to a small concentration of the neutral 
Curve 751909-A

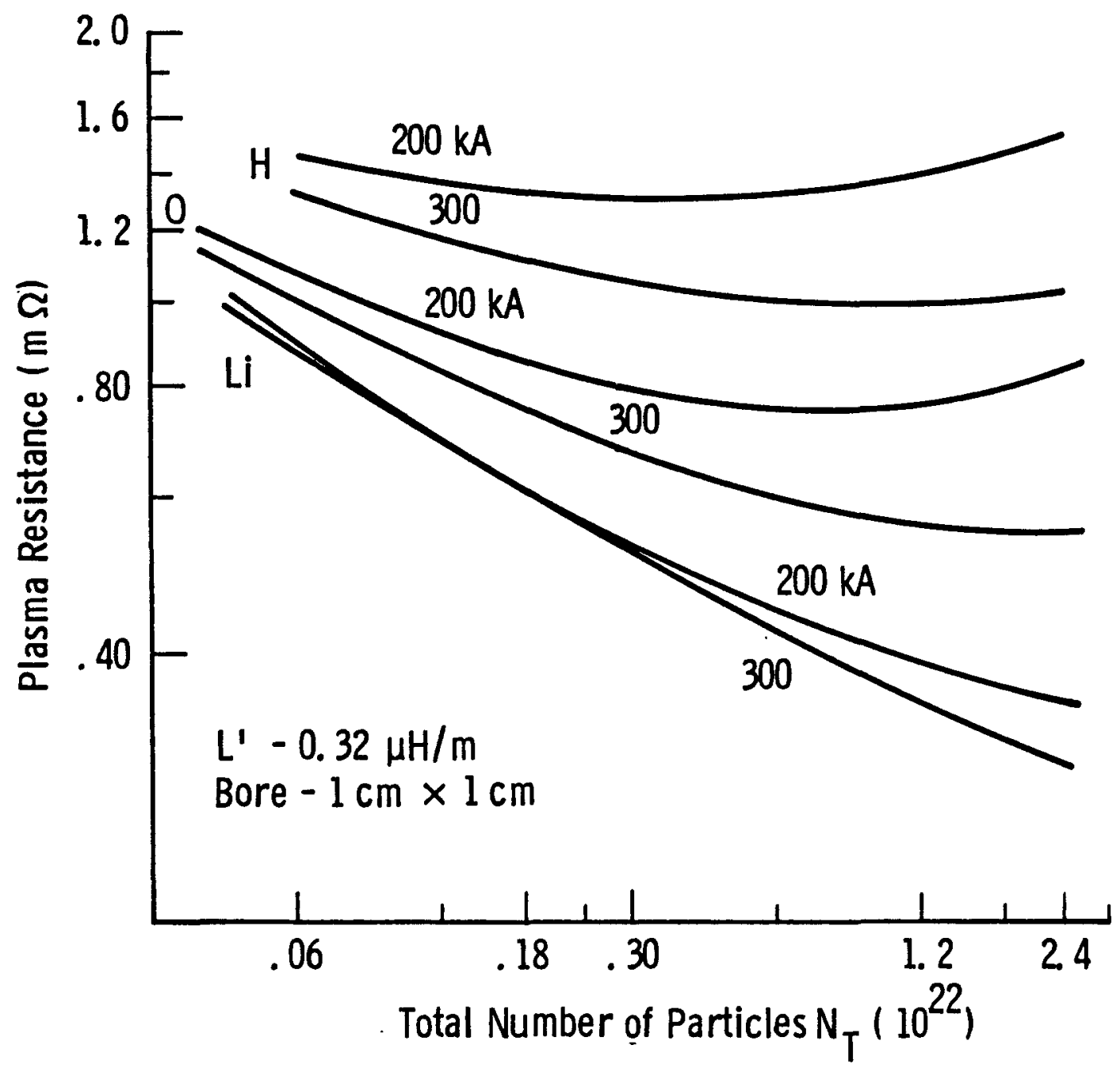

Figure 3.1 - Arc resistance versus the total number of particles (charged ions and neutrals) in the plasma armature for the case of a railgyn with an L' of $0.32 \mu \mathrm{H} / \mathrm{m}$ and a square bore of $1 \mathrm{~cm}^{2}$ in cross-section. 
Curve 751958-A

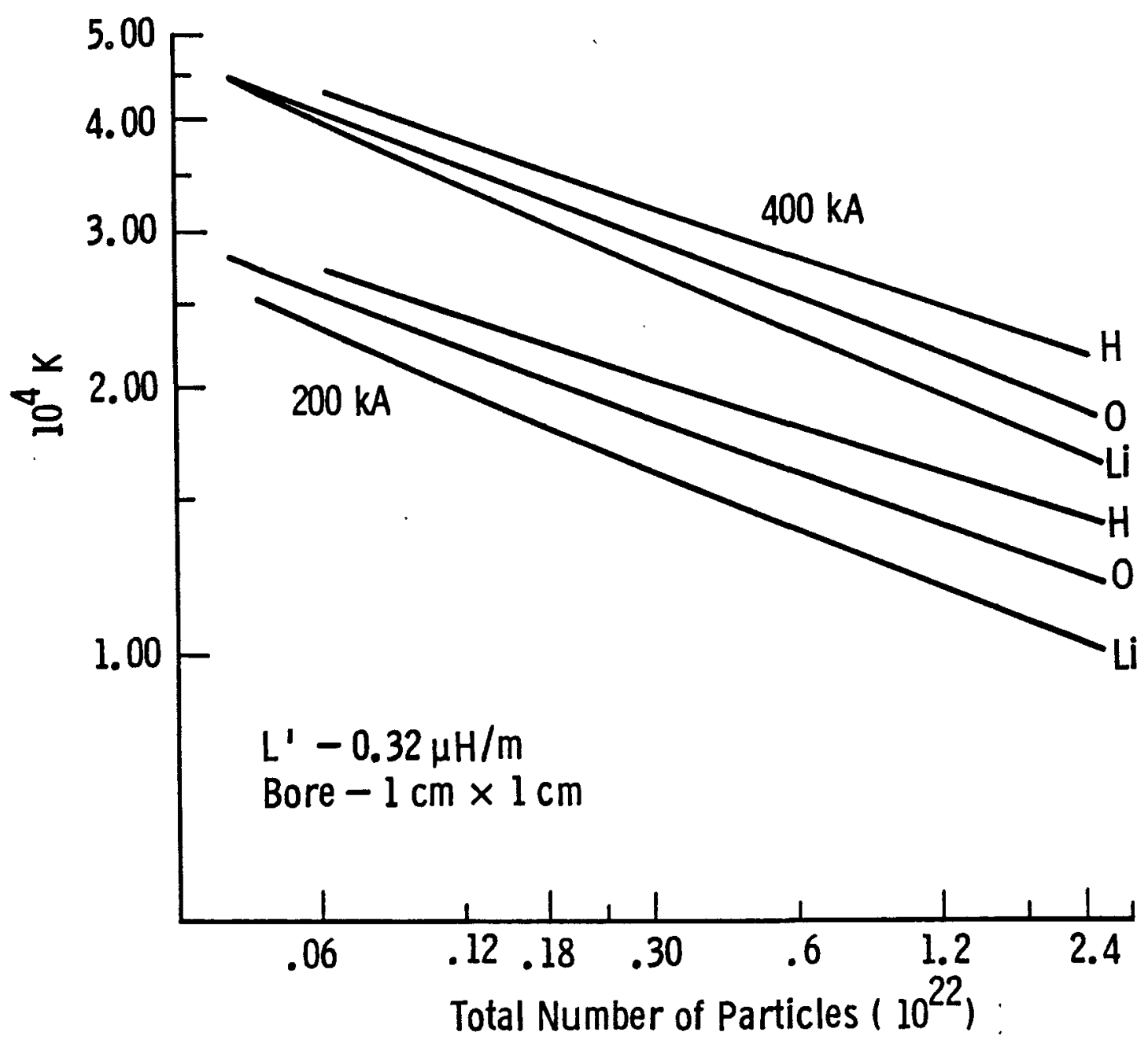

Figure 3.2 - Temperature of the plasma armature as a function of total number of particles in the armature for the case of a square-bore railgun $\left(1 \mathrm{~cm}^{2}\right)$ with an $L^{\prime}$ of $0.32 \mu \mathrm{H} / \mathrm{m}$. 
Curve 751959-A

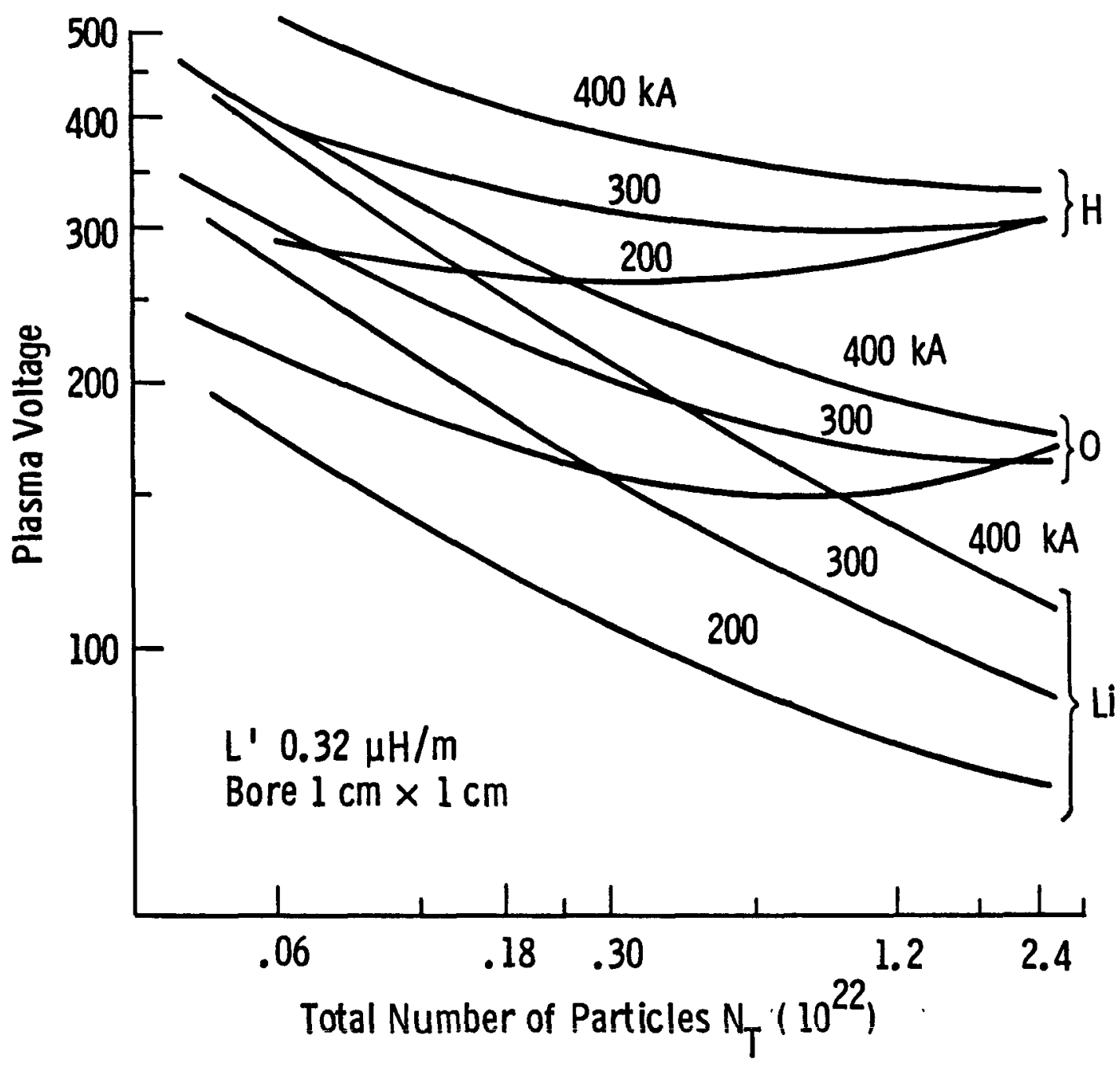

Figure 3.3 - Arc voltage versus the total number of particles (charged and neutral) in the plasma armature for the case of a railgun with an $L^{\prime}$ of $0.32 \mu \mathrm{H} / \mathrm{m}$ and a square bore of $1 \mathrm{~cm}^{2}$ in crosssection. 
Curve $751960-A$

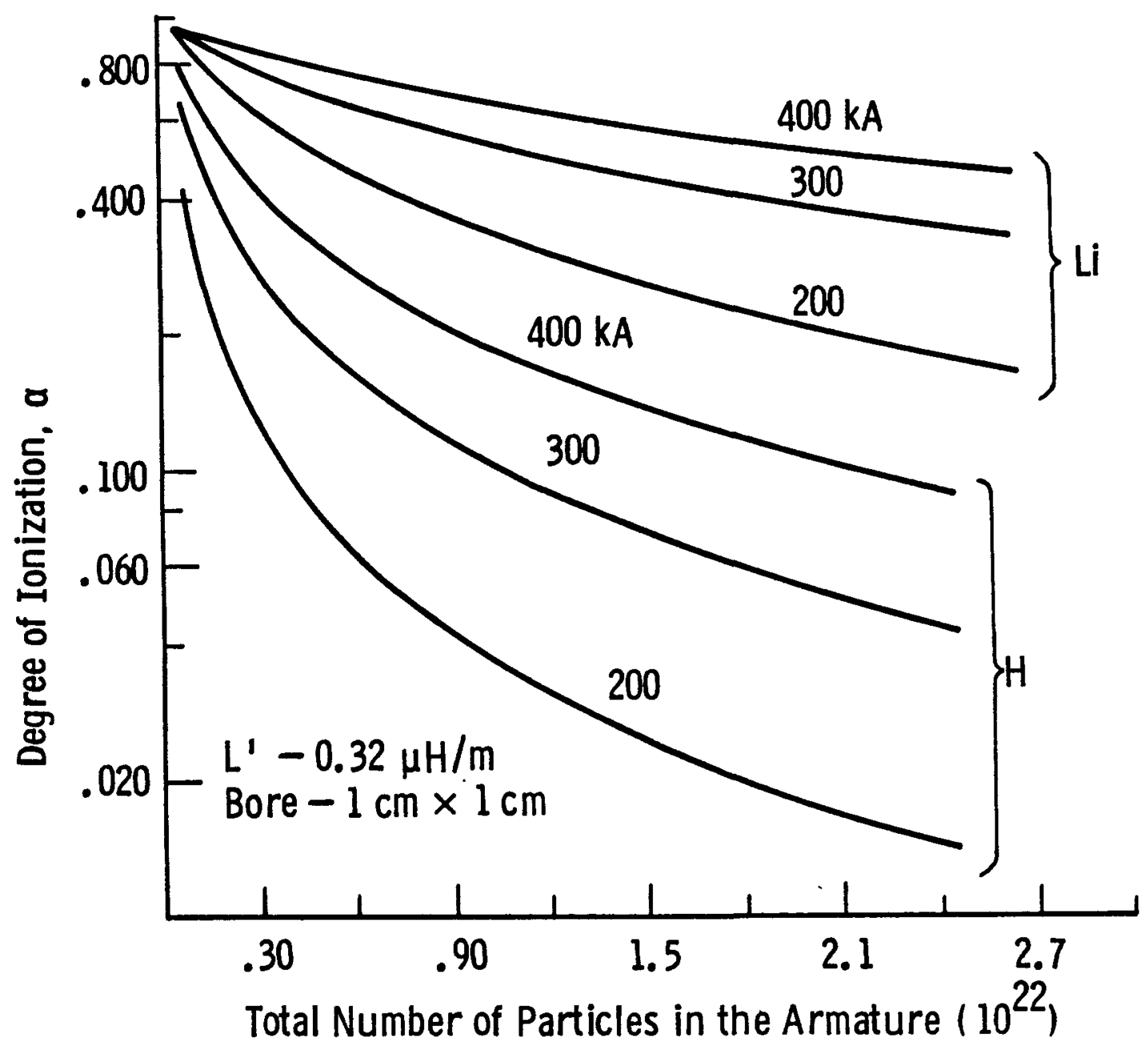

Figure 3.4 - Percentage of plasma ionization versus the total number of particles in the plasma armature. 
Curve 751957-A

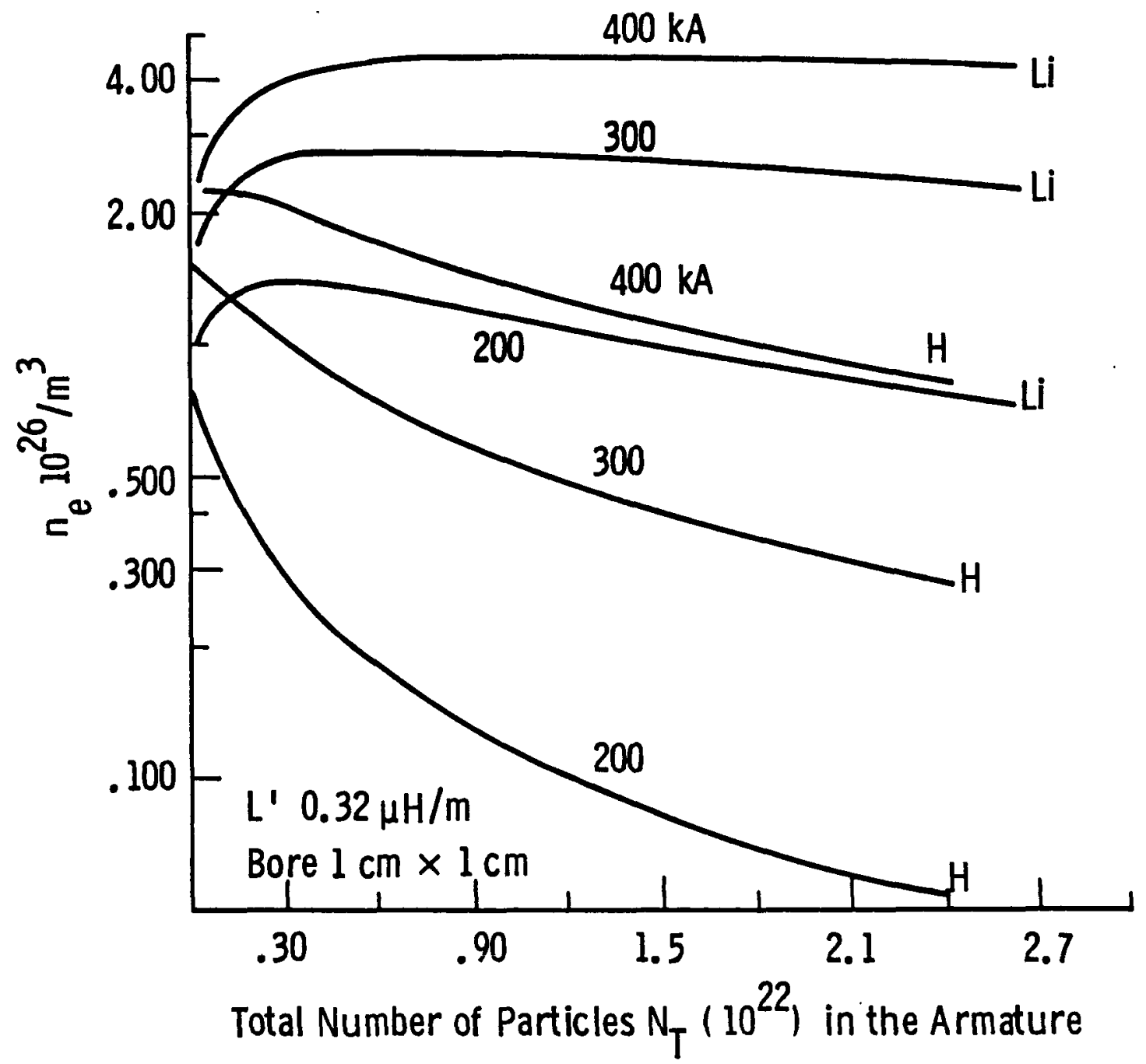

Figure 3.5 - Electron density in the plasma armature versus the total number of particles in the armature. 
particles. When these conditions prevail, the net resistance of the armature behaves almost independently of the current. As the current increases, the temperature increases and the resistivity drops. However, the length of the armature also decreases resulting in a smaller cross-sectional area through which the plasma current passes. The overall effect is to make the net resistance of the armature insensitive to variation in the current.

As the number of particles in the armature increases, the arc length grows, and the resistance generally decreases. In the case of a Li plasma armature in the gun used in the example, the armature resistance is reduced to a level below $0.3 \mathrm{mh}$ for a current of $300 \mathrm{kA}$ when the number of particles in the armature reaches a value of $2 x$ $10^{22}$. However, the plasma also cools with increasing number of particles. In the case of $H$ or 0 plasma, due to the high ionization potential of the atomic species, the degree of ionization in these plasmas decreases rapidly as the plasma cools. The consequence is the presence of a high concentration of neutral particles giving rise to a significant contribution from the electron-neutral scattering to the resistivity and consequently a higher resistance. Thus in these plasmas, the overall armature resistance at first decreases with the number of particles present in the armature until a point is reached when further increase in the number of particles makes the neutrals dominate the plasma resistivity. Beyond that point, the armature resistance increases with increasing number of particles.

For a pure 0 plasma at $300 \mathrm{kA}$, the resistance decreases from approximately $1.2 \mathrm{mn}$ for $5 \times 10^{20}$ particles to about $0.6 \mathrm{~m}$ for $1 \times 10^{22}$ particles in the armature. For a pure $H$ plasma at the same current the resistance appears to fall from $1.4 \mathrm{mn}$ to $1 \mathrm{~m} \Omega$ as the number of particles is increased from $5 \times 10^{20}$ to $1 \times 10^{22}$. When the number of particles increases beyond $2 \times 10^{22}$, the armature resistance begins to gradually increase. 
With reference to Figure 3.2 and 3.6 , we see that the armature temperature and more importantly the radiation intensity decreases monotonically with increasing number of particles.

At $200 \mathrm{kA}$, when the number of particles increases from $5 \times 10^{20}$ to $2 \times 10^{22}$, the temperature of a Li plasma drops from a $25,000 \mathrm{~K}$ to $12,000 \mathrm{~K}$. The corresponding radiation intensity from the armature falls from $2.5 \mathrm{MW} / \mathrm{cm}^{2}$ to below $0.1 \mathrm{MW} / \mathrm{cm}^{2}$. For an 0 plasma armature, the temperature decreases from approximately $27,000 \mathrm{~K}$ to about $13,000 \mathrm{~K}$ and the radiation intensity goes from $3 \mathrm{MW} / \mathrm{cm}^{2}$ to about $0.14 \mathrm{MW} / \mathrm{cm}^{2}$. For a $\mathrm{H}$ plasma armature, the temperature decreases from about $30,000 \mathrm{~K}$ to about $16,500 \mathrm{~K}$ and the radiation intensity drops from about $5 \mathrm{MW} / \mathrm{cm}^{2}$ to approximately $0.4 \mathrm{MW} / \mathrm{cm}^{2}$.

At $400 \mathrm{kA}$, when the number of particles increases from $5 \times 10^{20}$ to $2 \times 10^{22}$, the temperature of a Li plasma drops from a $45,000 \mathrm{~K}$ to $18,000 \mathrm{~K}$. The corresponding radiation intensity from the armature falls from $25 \mathrm{MW} / \mathrm{cm}^{2}$ to below $0.5 \mathrm{MW} / \mathrm{cm}^{2}$. For an 0 plasma armature, the temperature decreases from approximately $45,000 \mathrm{~K}$ to about $20,000 \mathrm{~K}$ and the radiation intensity goes from $25 \mathrm{MW} / \mathrm{cm}^{2}$ to about $0.8 \mathrm{MW} / \mathrm{cm}^{2}$. For a $\mathrm{H}$ plasma armature, the temperature decreases from about $47,000 \mathrm{~K}$ to about $23,000 \mathrm{~K}$ and the radiation intensity drops from about $30 \mathrm{MW} / \mathrm{cm}^{2}$ to approximately $1.2 \mathrm{MW} / \mathrm{cm}^{2}$.

Even refractory and conducting materials such as tungsten or molybdenum are unable to survive the radiation from the armature for more than a few $\mu s$ when the radiation intensity exceeds $1 \mathrm{MW} / \mathrm{cm}^{2}$. For radiation intensity below $1 \mathrm{MW} / \mathrm{cm}^{2}$, there is a chance for these materials to survive if the dwell time of the plasma armature is of the order of several $\mu \mathrm{s}$. The thermal conductivity of the materials plays an important part. In the next section, the radiative heating of the wall materials is analysed. 
Curve 751911-A

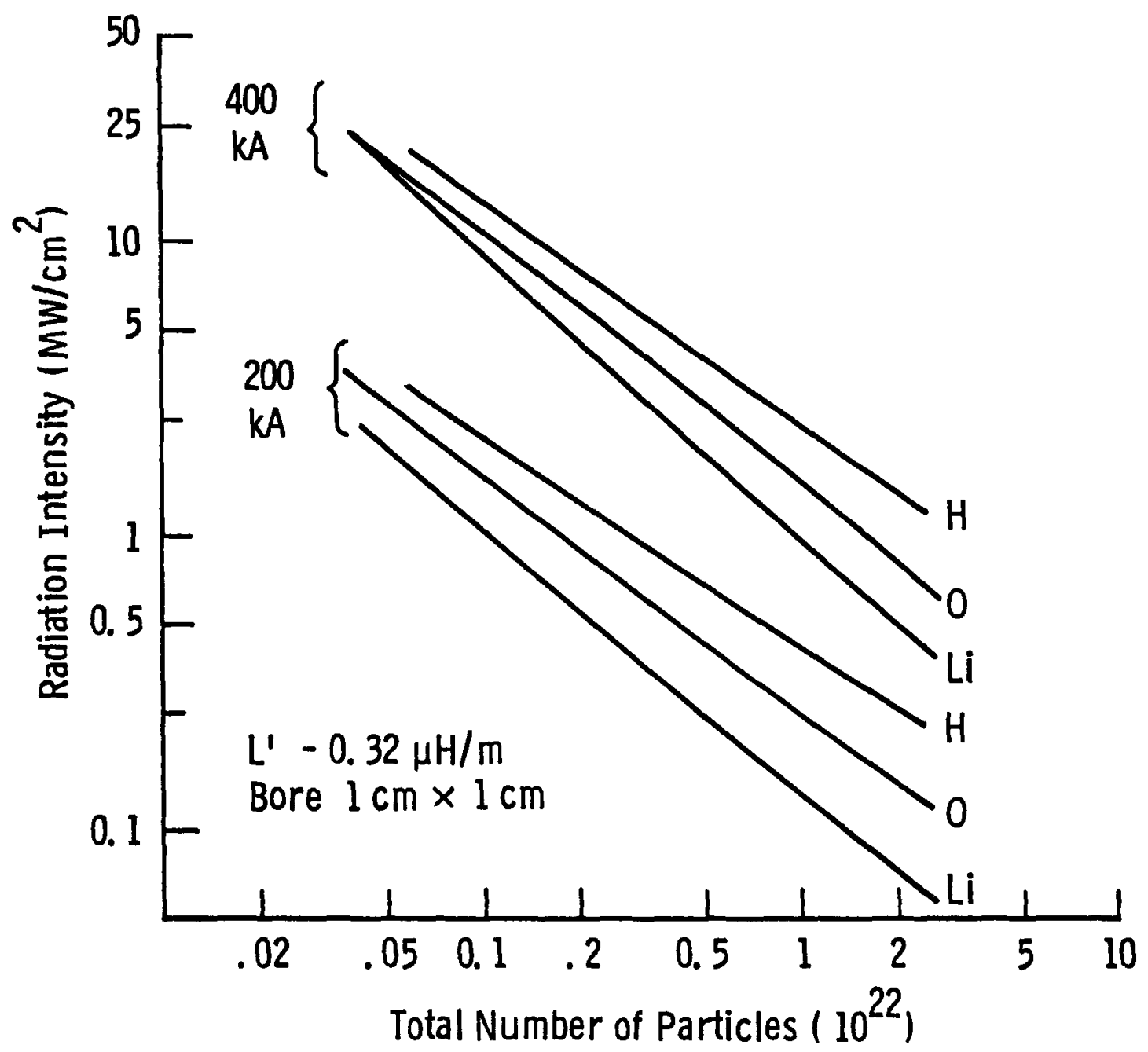

Figure 3.6 - Radiation intensity versus total number of particles in the plasma armature for the case of a square-bore railgun with a bore cross-section of $1 \mathrm{~cm}^{2}$ and an $L^{\prime}$ of $0.32 \mu \mathrm{H} / \mathrm{m}$. 


\subsection{PLASMA RADIATIVE HEATING OF THE MALL MATERIALS}

The wall of the bore is subjected to an incident radiative flux given by,

$$
Q=\epsilon \sigma_{S} T_{A}^{4}
$$

where $T_{A}$ is the arc temperature, $\sigma_{s}$ is the Stefan constant, and $\epsilon$ is the effective emissivity. We shall assume unity for the effective emissivity for want of exact experimental data. This will tend to overestimate the radiative flux. The temperature $T_{A}$ strictly should be the temperature of the arc at a point which is approximately one optical (Rosselande) path length from the wall. Again, we can obtain a worstcase estimate by using the arc column temperature calculated from the model as the value for $T_{A}$.

Before the onset of melting, the wall temperature increases according to the standard equation for heat conduction in solid,

$$
\text { - } \rho c_{v} \frac{\partial T}{\partial t}=\frac{\partial}{\partial x}\left[\kappa \frac{\partial T}{\partial x}\right]
$$

where $\rho$ is the mass density, $c_{v}$ is the specific heat per unit mass of the rail, $x$ is its thermal conductivity, and $x$ is the displacement into the wall perpendicular to its surface. The boundary condition at the wall surface $(x=0)$ providing the radiative heat flux is,

$$
\frac{\partial T}{\partial x}=-Q=-\epsilon \sigma_{s} T^{4}
$$

Equation (3.2) with the boundary condition (3.3) can be solved to give the temperature at a point $x$ and time to be,

$$
\begin{gathered}
T(x, t)=T_{0}+Q\left\{(\pi \lambda)^{-1} \exp \left(-\lambda^{2} x^{2}\right)-x \operatorname{Erfc}(\lambda x)\right\} / \kappa \\
\lambda=\left(\rho c_{v} / 4 \pi t\right)^{1 / 2}
\end{gathered}
$$


where $T_{0}$ is the initial temperature of the rail. At the rail surface, $x=0$, the temperature at time $t$ is,

$$
T(0, t)=T_{0}+2 Q\left(t / \pi \rho c_{v} \kappa\right)^{1 / 2}, \quad t<t_{r}
$$

In the above, the time $t$ is measured from the instant the plasma armature first reaches the point of interest. The above expression applies only during the fly-by of the plasma armature. The dwell time of the plasma is indicated as $t_{r}$. After this time, the wall temperature would then fall off. It can be shown that, after the armature fly-by, the surface temperature of the wall at time $t>t_{r}$ is given by

$$
T(0, t)=T_{0}+2 Q\left\{t^{1 / 2}-\left(t-t_{r}\right)^{1 / 2}\right\} /\left(\pi \rho c_{v} k\right)^{1 / 2}
$$

The above heating of the wall by the plasma radiation applies to both the rail and the insulator. For the rail, an additional heating mechanism needs to be included: the magnetic heating (or commonly called Joule heating) of the rails. The magnetic and radiative heating of the rails strictly are a coupled thermal process. In a recent paper, Powell[19] treats this coupled phenomenon by carefully formulating a 1-D model in which the equations governing the velocity-skin effect (magnetic) heating of the rail is solved simultaneously with the equations for the radiative transfer. As also noted by Powell, there exist at present a high degree of uncertainty between application of the theoretical results and interpretations of experimental observations in any exact form. In view of this uncertainty, it appears that a consistent degree of accuracy can still be obtained even if we seek a simplification of the mathematical problem by un-coupling the magnetic heating of the rail from the radiative heating. In this procedure, we adopt a worst-case approach in estimating the heating effects from each of these contributions. By treating the combined effects as a superposition of the separate effects, the overall estimate for the 
temperature rise in the rails can reasonably be expected to be conservative in the maximal sense. This simplification allows rapid parametric studies over a wide range of plasma and rail parameters to be performed. The approach is in essence equivalent to a lump-parameter method.

\subsection{MAGNETIC (OR JOULE) HEATING OF THE RAILS}

The precise way of taking account of magnetic heating of the rails is to solve the coupled set of partial differential equations governing strong magneto-thermal diffusion in a conductor, coupled to the electrodynamics of the moving arc. An upper bound to the temperature rise due to magnetic heating, however, can be obtained as follows. The magnetic field within the plasma armature decreases from the breech-end of the armature to the muzzle-end of the armature. During the fly-by of the plasma armature, at a given station on the surface of the rail, one would see a magnetic field increasing monotonically with time. In the case of an uniform current density model, this monotonicity degenerates into one of linearity. After the plasma armature has completely passed by, the magnetic field takes on the value of the field produced by the constant current flowing in the rails. The temperature rise due to this temporal monotonic increasing variation of magnetic field with a plateau amplitude after a certain lapse of time is always less than that due to a sudden switch-on of a magnetic field with the same plateau amplitude. For a conservative (maximal) estimate of the rail surface temperature due to Joule heating, we may therefore use the result for the case of a sudden switch on of a magnetic field.

For the sudden switch-on of a magnetic field, the peak temperature rise occurs at the conductor surface and at the instant of switching on the field, if effects associated with temperature-dependent material properties were neglected. 
The question remains whether 2-D or even 3-D effects are important in determining the magneto-diffusion heating effects in the case of finite and curved rails. It can be shown that, in a sufficiently short time scale and in a sufficiently thin layer of the rail close to the surface, 2-D or 3-D magneto-diffusion may be ignored. In particular, the peak surface temperature which occurs at $t=0$ may be obtained from a solution of a 1-D magneto-diffusion problem:

$$
\begin{gathered}
\mu \frac{\partial H}{\partial x}=\frac{\partial}{\partial x}\left[\eta \frac{\partial H}{\partial x}\right] \\
\rho c_{v} \frac{\partial T}{\partial x}=\frac{\partial}{\partial x}\left[\kappa \frac{\partial T}{\partial x}\right]+\eta\left[\frac{\partial H}{\partial x}\right]
\end{gathered}
$$

with the appropriate boundary conditions, where $\mu$ is the magnetic permeability, $\eta$ is the rail resistivity and the other symbols have their usual meaning. Again, locally and for the very short time of interest, the boundary conditions can be chosen to be the same as those for a semi-infinite conducting half-space, with the surface of the half-space corresponding to the rail surface. This is the problem solved by Kidder [25] and re-solved by several others e.g. [26]. From their solution, the maximum surface temperature rise can be given as,

$$
\Delta T=\frac{4}{\pi \rho c_{v}}\left[\frac{1}{2} \mu H_{\circ}^{2}\right] \ln \left[1+\frac{\pi}{2}\left(\frac{\rho c_{v} \eta}{2 \mu x}\right)^{1 / 2}\right]
$$

We use this expression to provide the upper bound to the magnetic heating of the rail due to the moving plasma armature.

\subsection{MINIMUM ARMATURE VELOCITY FOR WALL SURVIVABILITY}

If the plasma armature enters a barrel section with an entry velocity $v_{0}$ and is subjected to a constant acceleration $a$, the dwell time $t_{r}$ of the armature at the entry point is given by, 


$$
t_{r}=\left\{\left(v_{0}^{2}+2 a 1\right)^{1 / 2}-v_{0}\right\} / a
$$

where 1 is the length of the plasma armature which is obtained from solving the plasma model as described in Thio and Frost[24]. For a constant acceleration, the armature dwell time decreases with increasing entry velocity $v_{0}$. In the case of the rail, the total temperature rise is given by,

$$
\Delta T_{\text {rail }}=\Delta T_{r}+\Delta T_{J}
$$

where $\Delta T_{r}$ is the temperature $r i s e$ due to radiative heating given by Expression (3.5), and $\Delta T_{J}$ is the temperature rise due to Joule (magnetic) heating given by Expression (3.9). In the case of the insulator, the total temperature rise consists of only the radiative term. From Expression (3.5), we see that the total temperature rise of the wall decreases with decreasing armature dwell time. In order to control the wall temperature rise to be below a certain specified value, the armature dwell time is required to be below a certain value. In turn, this requires a certain entry velocity for a given acceleration of the armature. Substituting Expression (3.10) for the dwell time in the radiative heating Expression (3.5), and introducing the resultant Expression in (3.11) and using the Kidder's temperature Expression (3.9) if neccessary in Expression (3.11), an equation is obtained for the minimum entry velocity for a given acceleration, or a minimum acceleration for a given entry velocity, to limit the wall temperature rise to a specified value.

Let $T_{m}$ be the melting point of the wall material and $T_{0}$ its initial temperature. The maximum allowable temperature rise at the wall surface before onset of melting is given by $\Delta T=T_{m}-T_{0}$. In practice, because of thermal softening of the wall material at elevated temperature and the requirement to withstand the high pressure of the plasma armature, the allowable temperature rise for the wall is 
considerably lower than is given by the above expression based upon the melting point in order to avoid significant damage of the wall material.

Figure 3.7 shows the result of solving the resultant equation for minimum entry velocity to control the temperature rise in the tungsten rails to below $2500 \mathrm{~K}$ for a railgun with $1-\mathrm{cm}$ square bore, $0.32 \mu \mathrm{H} / \mathrm{m}$, and a $1-\mathrm{g}$ projectile. The calculations are made for the 3 different single-element plasmas, $L i, O$ and $H$, and for different values of current, $200 \mathrm{kA}$ and $300 \mathrm{kA}$. The minimum entry velocity is shown versus the total number of particles in the armature.

At $300 \mathrm{kA}$ for this gun, the required velocity for the armature to avoid rail damage can be seen to be very high (exceeding $50 \mathrm{~km} / \mathrm{s}$ ) for all the 3 different plasmas when the armature contains less than $10^{21}$ particles. Only when the armature contains more than $10^{22}$ particles does the minimum armature velocity fall below $20 \mathrm{~km} / \mathrm{s}$ in the case of a $H$ plasma armature, and below $10 \mathrm{~km} / \mathrm{s}$ in the case of an 0 plasma. In the case of a $\mathrm{Li}$ plasma, the calculation indicates that an entry velocity as low as $1 \mathrm{~km} / \mathrm{s}$ would be sufficient to avoid radiative damage if more than $10^{22}$ particles are present in the plasma armature.

At $200 \mathrm{kA}$, the situation improves markedly. With more than $10^{22}$ particles in the armature, the tungsten rails appear to be able to survive the armature radiation for moderate armature velocity for all the 3 different plasmas. Specifically, for a $H$ plasma a minimum armature velocity of approximately $5 \mathrm{~km} / \mathrm{s}$ is required, and for both 0 and $\mathrm{Li}$ plasmas, an entry velocity of the order of $100 \mathrm{~m} / \mathrm{s}$ appears to be all that is neccessary to avoid radiative damage. On the other hand, if only $10^{21}$ particles are used, entry velocity of $8 \mathrm{~km} / \mathrm{s}, 10 \mathrm{~km} / \mathrm{s}$, and $20 \mathrm{~km} / \mathrm{s}$ are required for a $\mathrm{Li}, O$ and $H$ plasma armature respectively. 


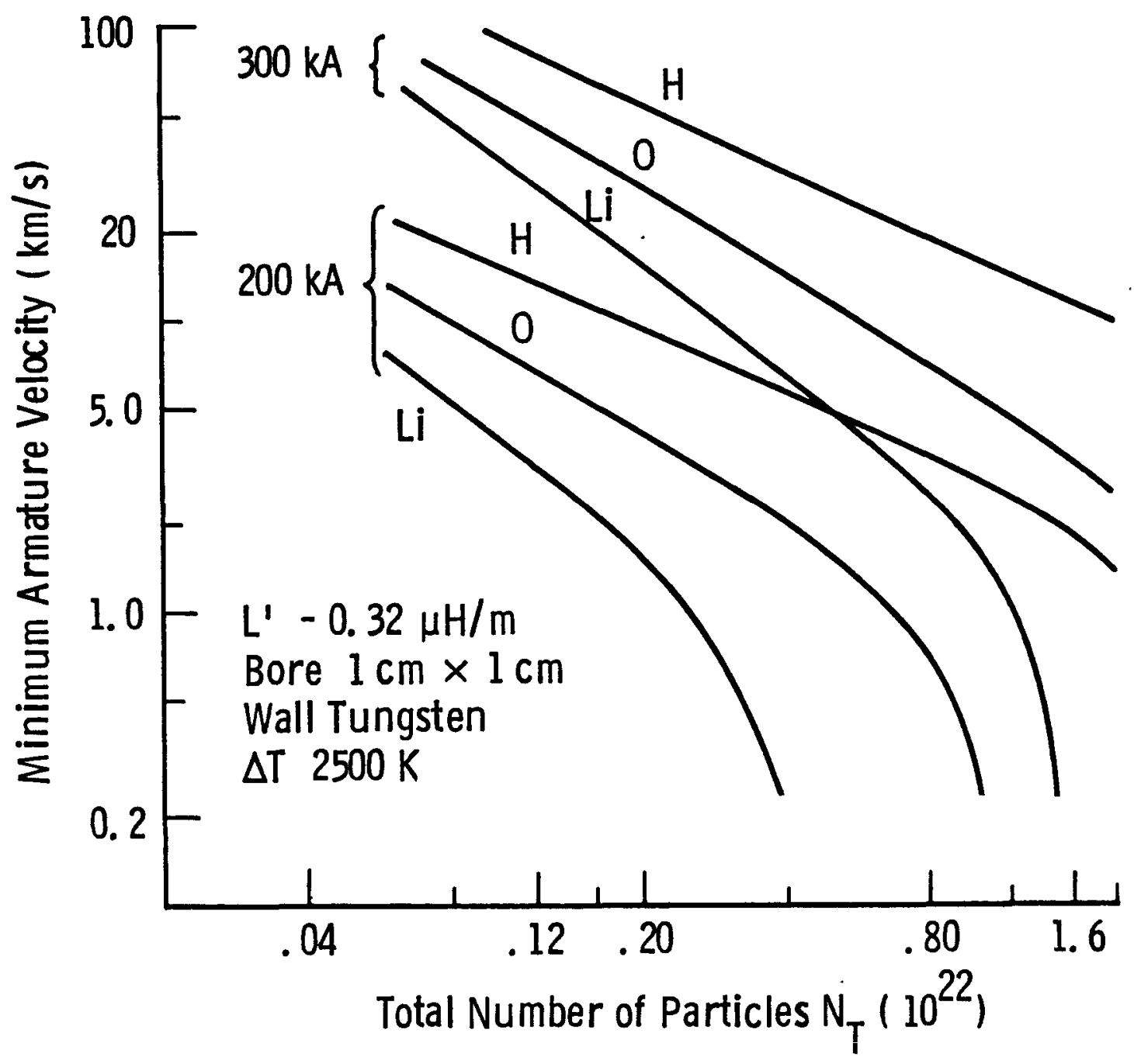

Figure 3.7 - Minimum armature velocity to limit the temperature rise in a tungsten rail to below $2500^{\circ} \mathrm{K} f \mathrm{~g}^{r}$ a square-bore railgun with a bore cross-section of $1 \mathrm{~cm}^{2}$ and a $L^{\prime}$ of $0.32 \mu \mathrm{H} / \mathrm{m}$. 


\subsection{CONCLUSION}

On the one hand, the modelling results show that the arc radiation is sufficiently high to cause ablation under the conditions of most railgun experiments conducted in the past. Under the same conditions the arc resistance also has a tendency towards a rather high value $(>1 \mathrm{mn})$. These properties were a consequence of operating the plasma armature under less than optimum conditions. In particular, the plasma armature in these experiments were initiated with less than the required number of particles. As a result, the initial plasma is very hot, emits radiation at a sufficiently high intensity, and begins to ablate the wall materials. Also, because of insufficient number of particles, the arc has a short length giving rise to a high resistance.

On the other hand, the modelling results indicate that there indeed exist domains of operating parameters in which radiative ablation of the wall materials can be completely eliminated. For a given set of gun parmeters and a given current, we can generally find a combination of number of particles in the armature and the armature velocity by which radiative ablation would not occur. We find, indeed, that by using a low atomic weight, low ionization ionic species such as $L i$, the armature velocity could be as low as a few $\mathrm{km} / \mathrm{s}$ when radiative ablation can be totally avoided under typical railgun operating conditions, and that the mass of the lithium armature required to initiate the arc remains below $10 \%$ of the projectile mass. The modelling results indicate that elimination of radiative ablation is indeed feasible, at least theoretically.

Based upon this theoretical suggestion, we planned to initiate plasma armatures in our experiments from a mixture of fast burning chemical propellant and an easily dissociable lithium compound. 


\section{Armature Stability or Instability}

\subsection{BACKGROUND}

Thio[27], Powell and Batteh [28] have developed steady-state models of railgun plasma armatures. The general starting point in their models is the basic magnetogasdynamic equation

$$
\rho \frac{D \underline{v}}{D t}=-\nabla p+i \times \underline{B}
$$

By assuming a slug motion for the plasma armature and assuming that the acceleration is constant

$$
\frac{D v}{D t}=\underline{a}=\text { constant }
$$

The above equation (1) reduces to

$$
\rho \underline{\underline{a}}=-\nabla p+j \times B
$$

thereby el iminating the time dependence.

This steady-state approach has been very fruitful, and Thio[7] has used it to advantage in developing an understanding of the radiative damage to the rails and insulators in the barrel, and in developing the basic concepts for the controlled generation of plasma armature (propellant and light alkali compounds initiated plasma armatures) and the mechanically contained plasma armatures (See Section 6).

The applicability of the steady-state models, however, is limited by the growth of instabilities of the plasma armature. The 
purpose of this note is to examine one of the most fundamental instabilities associated with the plasma armature - that of RayleighTaylor - and to provide an estimate of its growth rate.

The time for the Rayleigh-Taylor instability to grow will determine the transition of the stable plasma armature to deflagration.

\subsection{DERIVATION OF THE PERTURBATION EQUATIONS}

Re-write Equation (4.1) in 1-D:

$$
\rho \frac{D v}{D t}=-\frac{\partial p}{\partial z}+j B
$$

We shall also need the continuity equation to ensure that the perturbed motion also satisfies mass conservation:

$$
\frac{\partial \rho}{\partial t}+\frac{\partial}{\partial z}(\rho v)=0
$$

Let the solution to (4.3) and (4.4) be expressed in terms of an asymptotic series

$$
v=\sum_{n=0}^{\infty} \epsilon^{n} v_{n}, \quad \rho=\sum_{n=0}^{\infty} \epsilon^{n} \rho_{n}, \quad p=\sum_{n=0}^{\infty} \epsilon^{n} p_{n}
$$

The first term of the asymptotic series satisfies the steady state equations. That is 


$$
\begin{gathered}
\rho_{0} \frac{D v_{0}}{D t}=-\frac{\partial p_{0}}{\partial z}+j B \\
\frac{\partial \rho_{0}}{\partial t}+\frac{\partial}{\partial z}\left(\rho_{0} v_{0}\right)=0 \\
\text { with } \frac{D v_{0}}{D t}=a=\text { constant } \equiv \frac{\partial v_{0}}{\partial t}+v_{0} \frac{\partial v_{0}}{\partial z}
\end{gathered}
$$

In this note, we shall only consider the asymptotic expansion up to order $\epsilon$ : That is, we shall write

$$
v=v_{0}+\epsilon v_{1}, \quad \rho=\rho_{0}+\epsilon \rho_{1}, \quad p=p_{0}+\epsilon p_{1}
$$

Then Equation (4.3) becomes

$$
\begin{gathered}
\left(\rho_{0}+\epsilon \rho_{1}\right)\left[\frac{\partial}{\partial t}\left(v_{0}+\epsilon v_{1}\right)+\left(v_{0}+\epsilon v_{1}\right) \frac{\partial}{\partial z}\left(v_{0}+\epsilon v_{1}\right)\right] \\
=-\frac{\partial p_{0}}{\partial z}-\epsilon \frac{\partial p_{1}}{\partial t}+j B
\end{gathered}
$$

We now expand the lefthand side of Equation (4.9) as follows:

$$
\begin{aligned}
\text { L.H.S. }(4.9) & =\left(\rho_{0}+\epsilon \rho_{1}\right)\left\{\frac{\partial v_{0}}{\partial t}+\epsilon \frac{\partial v_{1}}{\partial t}+\left(v_{0}+\epsilon v_{1}\right)\left[\frac{\partial v_{0}}{\partial z}+\epsilon \frac{\partial v_{1}}{\partial z}\right]\right\} \\
& =\left(\rho_{0}+\epsilon \rho_{1}\right)\left\{\frac{\partial v_{0}}{\partial t}+\epsilon \frac{\partial v_{1}}{\partial t}+v_{0} \frac{\partial v_{0}}{\partial z}+\epsilon\left[v_{1} \frac{\partial v_{0}}{\partial z}+v_{0} \frac{\partial v_{1}}{\partial z}\right]+0\left(\epsilon^{2}\right)\right\}
\end{aligned}
$$




$$
=\left(\rho_{0}+\epsilon \rho_{1}\right)\left\{\frac{\partial v_{0}}{\partial t}+v_{0} \frac{\partial v_{0}}{\partial z}+\epsilon\left[\frac{\partial v_{1}}{\partial t}+v_{1} \frac{\partial v_{0}}{\partial z}+v_{0} \frac{\partial v_{1}}{\partial z}\right]+0\left(\epsilon^{2}\right)\right\}
$$

Combining terms multiplied by $\rho_{0}$ and by $\epsilon$ gives

$$
\begin{aligned}
& \text { L.H.S. }(4.9)=\rho_{0}\left[\frac{\partial v_{0}}{\partial t}+v_{0} \frac{\partial v_{0}}{\partial z}\right] \\
& +\epsilon\left\{\rho_{1}\left[\frac{\partial v_{0}}{\partial t}+v_{0} \frac{\partial v_{0}}{\partial z}\right]+\rho_{0}\left[\frac{\partial v_{1}}{\partial t}+v_{1} \frac{\partial v_{0}}{\partial z}+v_{0} \frac{\partial v_{1}}{\partial z}\right]\right\} \\
& +0\left(\epsilon^{2}\right)
\end{aligned}
$$

Equating terms of equal order in $\epsilon$ in Equations (4.9) and (4.10), we get

$$
\begin{gathered}
\rho_{0}\left[\frac{\partial v_{0}}{\partial t}+v_{0} \frac{\partial v_{0}}{\partial z}\right]=-\frac{\partial p_{0}}{\partial z}+j B \\
\rho_{1}\left[\frac{\partial v_{0}}{\partial t}+v_{0} \frac{\partial v_{0}}{\partial z}\right]+\rho_{0}\left[\frac{\partial v_{1}}{\partial t}+v_{0} \frac{\partial v_{1}}{\partial z}+v_{1} \frac{\partial v_{0}}{\partial z}\right]=-\frac{\partial p_{1}}{\partial t}
\end{gathered}
$$

The first of these equations govern simply the unperturbed motion. In the second Equation (4.12), the group

$$
\frac{\partial v_{0}}{\partial t}+v_{0} \frac{\partial v_{0}}{\partial z}=a
$$

is simply the acceleration of the unperturbed motion. Also, by assuming that the unperturbed motion gives rise to the motion of the plasma armature as a slug,

$$
\begin{gathered}
\frac{\partial v_{0}}{\partial z}=0 \\
4-4
\end{gathered}
$$


Equation (4.12) then reduces to

$$
\rho_{0} \frac{\partial v_{1}}{\partial t}+v_{0} \frac{\partial v_{1}}{\partial z}+\frac{\partial p_{1}}{\partial z}+\rho_{1} a=0
$$

This is the equation which governs the momentum exchange in the perturbed motion.

Applying the same treatment to the continuity equation, we have

$$
\begin{gathered}
\frac{\partial\left(\rho_{0}+\epsilon \rho_{1}\right)}{\partial t}+\frac{\partial}{\partial z}\left(\rho_{0}+\epsilon \rho_{1}\right)\left(v_{0}+\epsilon v_{1}\right)=0 \\
\frac{\partial \rho_{0}}{\partial t}+\epsilon \frac{\partial \rho_{1}}{\partial t}+\frac{\partial}{\partial z}\left[\rho_{0} v_{0}+\epsilon\left(\rho_{1} v_{0}+\rho_{0} v_{1}\right)+0\left(\epsilon^{2}\right)=0\right. \\
\frac{\partial \rho_{0}}{\partial t}+\frac{\partial}{\partial z}\left(\rho_{0} v_{0}\right)+\epsilon\left[\frac{\partial \rho_{1}}{\partial t}+v_{0} \frac{\partial \rho_{1}}{\partial z}+\frac{\partial}{\partial z}\left(\rho_{0} v_{1}\right)\right]=0
\end{gathered}
$$

By virtue of Equation (4.7) for the unperturbed motion, we have for the perturbed motion

$$
\frac{\partial \rho_{1}}{\partial t}+v_{0} \frac{\partial \rho_{1}}{\partial z}+\frac{\partial}{\partial z}\left(\rho_{0} v_{1}\right)=0
$$

Equation (4.13) and (4.14) together govern the development of the perturbed motion or the instability of interest. We shall also need the equation of state for the plasma, which we assume to be

$$
p=(1+a) \frac{\rho k T}{m_{i}}
$$

where $k$ is the Boltzman constant, and $m_{i}$ is the mass per unit atom or ion of the atomic or ionic species of which the plasma armature is composed. Using Thio's approximation of uniform temperature and degree of ionization $\alpha$, we may write the equation of state as 
where

$$
p=k_{1} \rho
$$

$$
k_{1}=\frac{(1+a) k T}{m_{i}}
$$

Equation (4.13) can be rewritten as:

$$
\rho_{0}\left[\frac{\partial v_{1}}{\partial t}+v_{0} \frac{\partial v_{1}}{\partial z}\right]+k_{1} \frac{\partial \rho_{1}}{\partial z}+\rho_{1} a=0
$$

Equation (4.14) and (4.18) are a coupled pair of partial differential equations which need to be solved simultaneously to determine the growth of the instability.

\subsection{SOLVING THE PERTURBED EQUATIONS}

To solve the perturbed equations (4.14) and (4.18), we shall make the following natural transformation of variables (coordinates):

$$
\begin{gathered}
(z, t) \rightarrow\left(5, t^{\prime}\right) \\
z-v_{0} t=5 \\
t=t^{\prime}
\end{gathered}
$$


We regard henceforth all functions to be functions of $\left(s, t^{\prime}\right)$. Thus

$$
\begin{aligned}
\frac{\partial}{\partial t} f\left(S, t^{\prime}\right) & =\frac{\partial f}{\partial \zeta} \frac{\partial \zeta}{\partial t}+\frac{\partial f}{\partial t^{\prime}} \frac{\partial \zeta}{\partial t} \\
& =-v_{0} \frac{\partial f}{\partial \zeta}+\frac{\partial f}{\partial t^{\prime}}
\end{aligned}
$$

and

$$
\begin{aligned}
\frac{\partial}{\partial z} f\left(\zeta, t^{\prime}\right) & =\frac{\partial f}{\partial \zeta} \frac{\partial \zeta}{\partial z}+\frac{\partial f}{\partial t^{\prime}} \frac{\partial t^{\prime}}{\partial z} \\
& =\frac{\partial f}{\partial \zeta}
\end{aligned}
$$

Using (4.20) and (4.21), Equations (4.14) and (4.18) can be recast into the following forms:

$$
\begin{aligned}
& \frac{\partial \rho_{1}}{\partial t^{\prime}}+\frac{\partial}{\partial S}\left(\rho_{0} v_{1}\right)=0 \\
& \rho_{0} \frac{\partial v_{1}}{\partial t^{2}}+k_{1} \frac{\partial \rho_{1}}{\partial S}+\rho_{1} a=0
\end{aligned}
$$

Applying the operator $\frac{\partial}{\partial t}$, to Equation (4.23), we obtain:

$$
\rho_{0} \frac{\partial^{2} v_{1}}{\partial t^{2}}+k_{1} \frac{\partial}{\partial \zeta}\left(\frac{\partial \rho_{1}}{\partial t^{2}}\right)+a \frac{\partial \rho_{1}}{\partial t^{2}}=0
$$

Note that

$$
\frac{\partial \rho_{0}}{\partial t^{\prime}}=0
$$

Substituting Equation (4.22) into (4.24), we obtain

4-7 


$$
\rho_{\circ} \frac{\partial^{2} v_{1}}{\partial t^{2}}-k_{1} \frac{\partial^{2}}{\partial \zeta^{2}}\left(\rho_{0} v_{1}\right)-a \frac{\partial}{\partial \zeta}\left(\rho_{0} v_{1}\right)=0
$$

or

$$
k_{1} \frac{\partial^{2} f}{\partial s^{2}}+a \frac{\partial f}{\partial \zeta}-\frac{\partial^{2} f}{\partial t^{2}}=0
$$

where

$$
f=\rho_{0} v_{1} .
$$

We can now analyze Equation (4.25) and examine the growth of the perturbed motion using the standard methods of modal analysis. Let

$$
f\left(s, t^{\prime}\right)=e^{s t+i p s}
$$

We take the real part of $f$ for the perturbed motion. Substituting the above mode for $f$ into equation (4.25) we obtain the relationship:

$$
k_{1}\left(-p^{2}\right)+a(i p)-s^{2}=0 .
$$

The growth rate of the instability is given by the real part of $s$. Solving for $s$, we get

$$
\begin{gathered}
s^{2}=i a p-k_{1} p^{2} \\
s= \pm i \sqrt{k_{1}} p\left(1-\frac{i a}{k_{1} p}\right)^{1 / 2} \\
= \pm i \sqrt{k_{1}} p\left[1-\frac{i a}{2 k_{1} p}+\frac{1}{8}\left[\frac{i a}{k_{1} p}\right]^{2}+\frac{1}{16}\left[\frac{i a}{k_{1} p}\right]^{3}+\ldots\right]
\end{gathered}
$$

At the boundaries of the plasma armature, the flow conditions require that the perturbed motion be such that 


$$
f\left(5, k^{\prime}\right) \int_{B d r y}=0
$$

This leads to the quantization of the modes:

$$
p=\frac{n \pi}{1}, n=1,2, \ldots, \ldots
$$

where $I$ is the stable length of the plasma armature obtained from the steady state solution. The real part of $s$ which governs the growth rate (the inverse of the characteristic time for the growth of the instability) can be obtained from Expression (4.27) as

$$
\begin{aligned}
\operatorname{Re}(s) & = \pm \frac{a}{2 \sqrt{k_{1}}}\left[1-\frac{1}{8}\left[\frac{a}{k_{1} p}\right]^{2}+0\left[\frac{a}{k_{1} p}\right]^{3}\right] \\
& \equiv 1 / \tau_{c}
\end{aligned}
$$

where $\tau_{c}$ is the time constant for the growth of the instability. The fastest growing mode of instability is the fundamental mode given by $n=$ 1 and

$$
p=\frac{\pi}{1}
$$

The characteristic time for this mode is the reciprocal of

$$
\operatorname{Re}\left(s_{1}\right)=\frac{a}{2 \sqrt{k_{1}}}\left[1-\frac{1}{8}\left(\frac{a l}{\pi k_{1}}\right)^{2}\right]
$$

or, substituting the definition of $k_{1}$ (4.17), we obtain for the characteristic time for the growth of the fundamental mode,

$$
t_{c}=\frac{2}{a}\left(\frac{(1+a) k_{B} T}{m_{i}}\right]^{1 / 2}\left\{1+\frac{1}{8}\left(\frac{a l}{\pi k_{1}}\right)^{2}+0\left(\frac{a l}{\pi k_{1}}\right)^{4}\right\}
$$

4-9 
We see from the above expression that we need low acceleration (low current for a given projectile mass), low atomic weight plasma species, and high temperature to obtain a high degree of stability (long growth time for any instability) for the armature. The last condition, high armature temperature, is contrary to the low armature temperature requirement to avoid radiative ablation.

To illustrate the implication of (4.31), consider the case of a plasma with a mean molecular weight of 14 , at a temperature of $20,000 \mathrm{~K}$, and which is $20 \%$ ionized. The characteristic time for the growth of instability may be calculated from the above to be approximately $340 \mu s$. This a typical time interval during which gram size projectiles have been accelerated to 2 to $4 \mathrm{~km} / \mathrm{s}$ in a typical small-bore railgun of approximately $1 \mathrm{~cm}$ in bore diameter. After this time period, the instability will grow and the armature will experience a transition from a localized arc to a state of deflagration. In that state, the armature may exist in several segments, or equivalently, the current could be distributed over an extended section of the launcher with several current density peaks. We are seeing here one of the processes by which secondary arcs may be formed. In the next chapter, we shall describe experiments in which we attempt to observe the existence and formation of these secondary arcs.

Though the following remark is not strictly relevant to our present consideration, it is worthwhile however to note in passing that, for low-velocity applications (such as tactical defense) where the required muzzle velocity $(2-5 \mathrm{~km} / \mathrm{s})$ is about 2 to 3 times lower than the sound speed ( 5 to $10 \mathrm{~km} / \mathrm{s}$ ) in a typical railgun plasma armature $10,000 \mathrm{~K}$ to $30,000 \mathrm{~K}$ ), the occurrence of secondary arcs are usually inconsequential to the interior ballistics of the projectile. This is so because the sum of the Lorentz forces on all the arc segments remains approximately the same, quite independent of the actual distribution of the current. If the sound speed in the gaseous medium is much greater than the projectile speed, most of the Lorentz force would eventually be 
transmitted to the projectile, minus the force required to accelerate the various segments of the arc against their inertia and viscous drag and any gaseous products in between. The losses incurred by the arc's inertia and viscous drag are typically small at low velocity. 


\section{Experiments}

\subsection{OVERVIEW}

Experimental investigations were carried out on the SUVAC launcher facility developed on this program (Figure 5.1). Details of the design and construction of the facility have already been described in previous reports $[23,29]$. The various phases of the facility development are summarised in Figure 5.2 .

Figures 5.3 and 5.4 , and Tables $5.1,5.2$, and 5.3 provides a quick overview of the progress of the experimental program.

\subsection{EXPERIMENTAL RESULTS SUMMARY}

\subsubsection{Group A}

Experimentation began in the middle of the program second year, 1984 September, with the testings of the novel plasma armature generation technique based upon the ignition of a mixture of chemical propellant and a lithium compound. A series of experiments (Group A in Table 5.1) was performed to establish a working composition of the plasma genertion package. Initially the plasma generation package typically consisted of a mixture of nitrocellulose and lithium nitrate powder, physically mixed and placed into a cylindrical container made from a thin aluminum foil. We found experimentally that a weight ratio of about 10:17:3 for nitrocellulose to lithium nitrate to aluminum works reasonably well. Later, we added lithium hydride to the mixture, and tested packages with weight ratios of nitrocellulose: I ithium nitrate: lithium hydride:aluminum around 5.0:3.5:3.5:1.5. The total mass of the package varies with each experiment, but generally the mass of mixture is chosen to produce about $10^{22}$ particles if all the chemical 


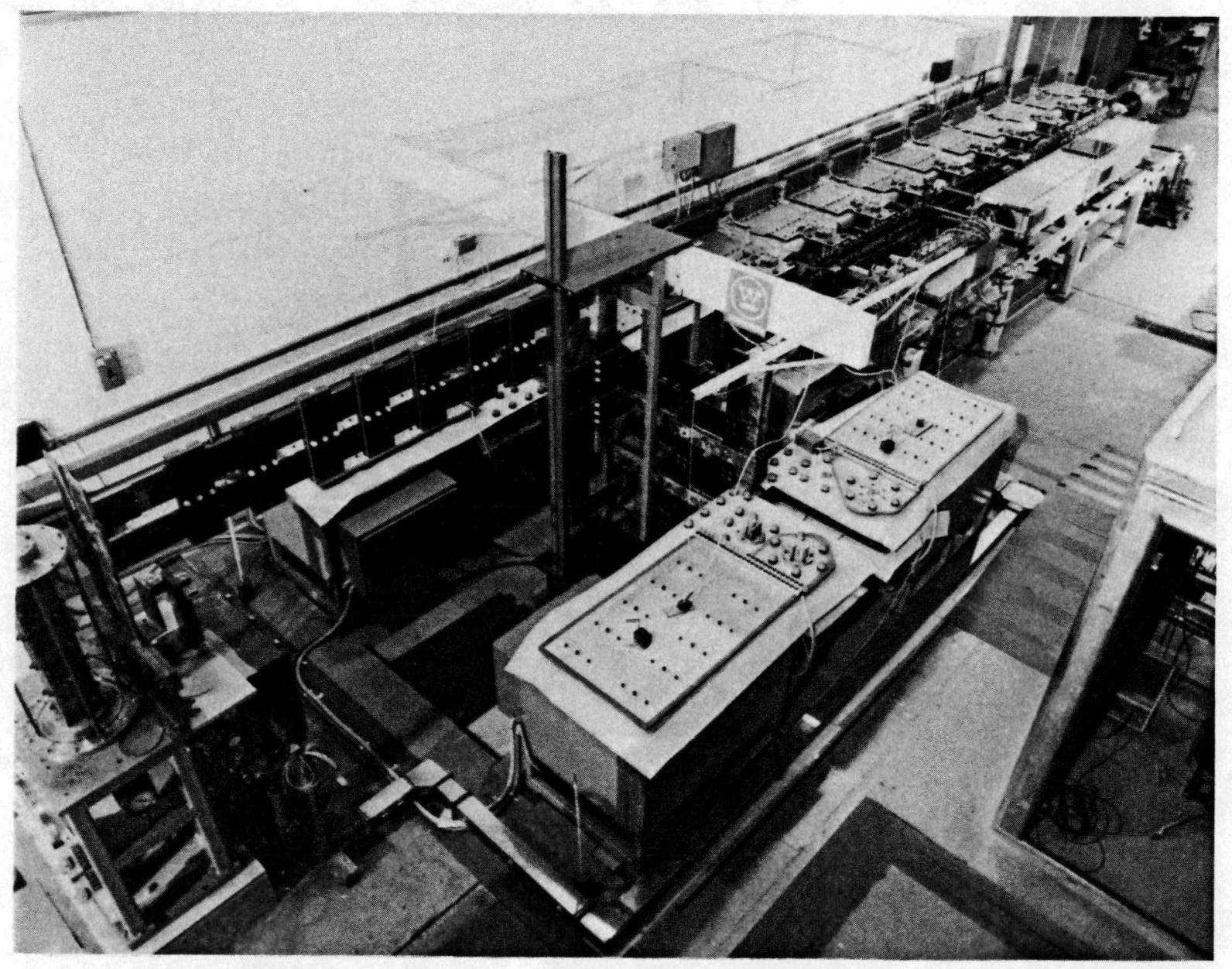

Figure 5.1 - Photograph of SUVAC Facility. 


\section{HARDWARE DEVELOPMENT PROGRAM}

\section{Power Supply}

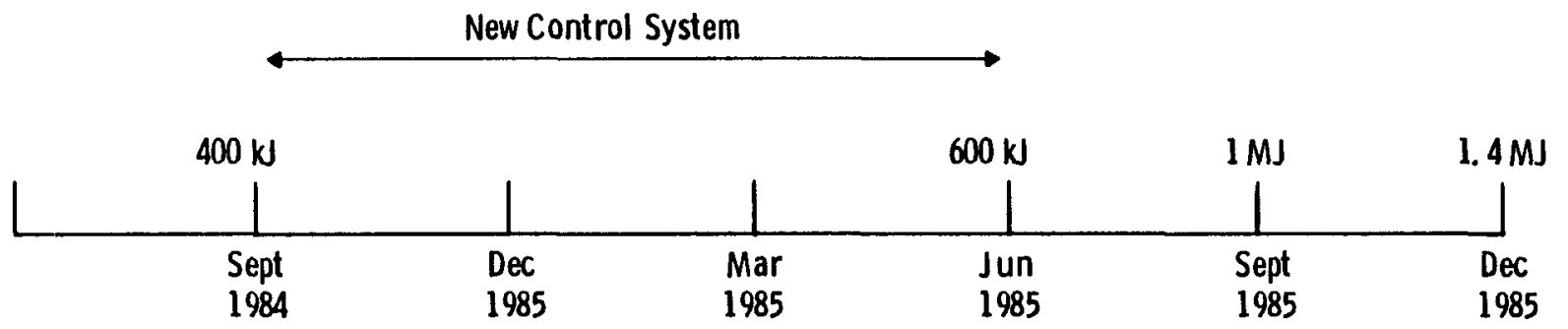

Data Acquisition System

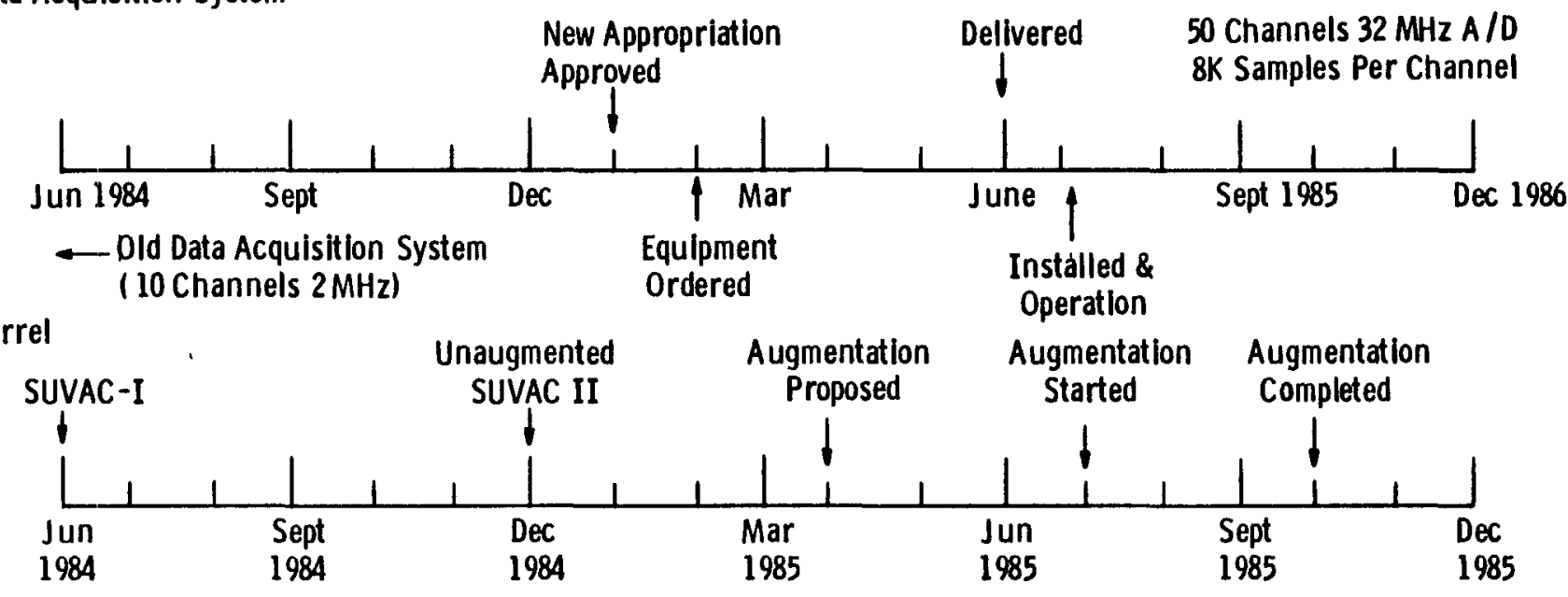

Figure 5.2 - Hardware Development Program. 


\section{EXPERIMENTAL PROGRAM ( I)}

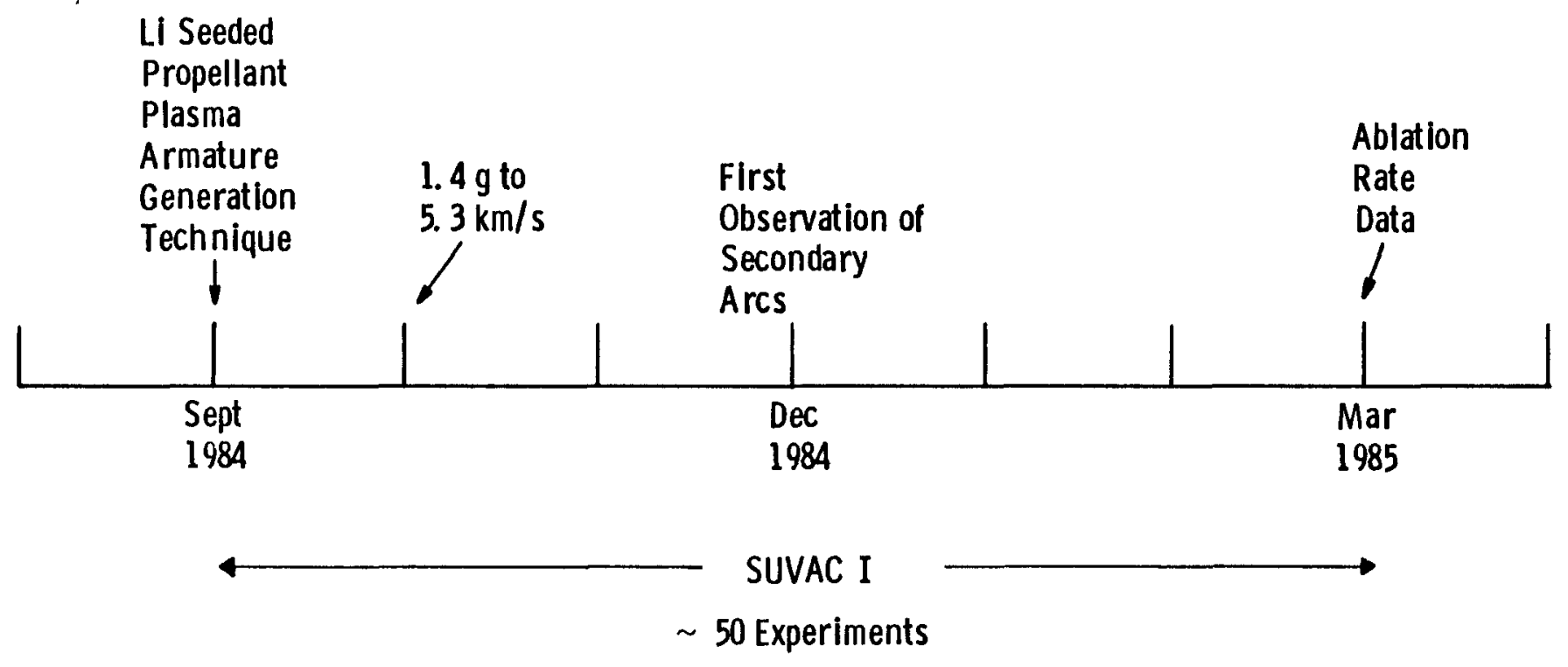

Figure 5.3 - Experimental Program (1). 
Dwg. 4278889

EXPERIMENTAL PROGRAM (2)

\begin{tabular}{lllll}
\multicolumn{3}{c}{ SUVAC-II Hardware Development } & & \\
DeC \\
1984
\end{tabular}

Figure 5.4 - Experimental Program (2). 
Table 5.1 - Experimental Summary I

\begin{tabular}{|c|c|c|}
\hline $\begin{array}{l}\text { Group } \\
\text { System }\end{array}$ & $\begin{array}{l}\text { A } \\
\text { 1-Stage, 6-ft. }\end{array}$ & $\begin{array}{l}\text { B } \\
1 \text {-stage, 6-ft }\end{array}$ \\
\hline $\begin{array}{l}\text { Test } \\
\text { Objectives }\end{array}$ & $\begin{array}{l}\text { Establish plasma } \\
\text { generation technique; }\end{array}$ & $\begin{array}{l}\text { Projectile development; } \\
\text { Develop diagnostics; }\end{array}$ \\
\hline $\begin{array}{l}\text { Critical/Limiting } \\
\text { Processes Under } \\
\text { Investigation }\end{array}$ & $\begin{array}{l}\text { Propellant burn rate; } \\
\text { Initial conductivity; } \\
\text { Rate of plasma expansion; } \\
\text { Uniformity of combustion; } \\
\text { Instabilities; }\end{array}$ & $\begin{array}{l}\text { Plasma blow-by; } \\
\text { Gouging; } \\
\text { Projectile compliance; } \\
\text { Projectile stability; } \\
\text { Material selection; }\end{array}$ \\
\hline $\begin{array}{l}\text { Number of } \\
\text { Experiments }\end{array}$ & 10 approx. & 15 approx. \\
\hline Remarks & $\begin{array}{l}\text { Working composition of } \\
\text { plasma generation package } \\
\text { developed }\end{array}$ & $\begin{array}{l}\text { Lexan finned projectile } \\
\text { with hemispheric cavity } \\
\text { at rear }\end{array}$ \\
\hline
\end{tabular}

Table 5.2 - Summary of Experiments II

Group

System

Test

Objectives
C

1-stage, 6-ft, augmented

Study augmented launcher behavior;

Develop diagnostics;

Critical/Limiting Armature Instability;

Processes Under Restrike;

Investigation

Number of

Experiments

Remarks Restrike;

8

$5.3 \mathrm{~km} / \mathrm{s}$ attained;

Significant reduction of rail damage by novel plasma armature $v$ isually noted.

\section{D}

1-stage, 6-ft unaugmented

Compare performance with augmented barrel of Group C; Collect ablation data;

Ablation;

Armature Instability;

Restrike;

12

Ablation < $12 \mathrm{~g} / \mathrm{MJ}$ achieved; Low ablation rate of new plasma armature confirmed, but not insignificant; Lower performance without augmentation as expected; First observation of secondary arcs made; 
Table 5.3 - Summary of Experiments III

\begin{tabular}{|c|c|c|}
\hline Group & $E$ & $\mathbf{F}$ \\
\hline System & 2-stage 12-ft SUVAC-II & 12-ft 1-stage SUVAC-II \\
\hline $\begin{array}{l}\text { Test } \\
\text { Objectives }\end{array}$ & Commissioning & $\begin{array}{l}\text { Behavior of 2-stage } \\
\text { launcher }\end{array}$ \\
\hline $\begin{array}{l}\text { Critical/Limiting } \\
\text { Processes Under } \\
\text { Investigation }\end{array}$ & $\begin{array}{l}\text { Firing equipment; } \\
\text { Inter-stage coupling; } \\
\text { Ground loops; } \\
\text { Inter-stage triggering; }\end{array}$ & $\begin{array}{l}\text { Ablation; } \\
\text { Viscous drag; } \\
\text { Secondary arcs; } \\
\text { Armature stability; }\end{array}$ \\
\hline $\begin{array}{l}\text { Number of } \\
\text { Experiments }\end{array}$ & 6 & 14 \\
\hline Remarks & $\begin{array}{l}\text { Overcome operating } \\
\text { problems; } \\
\text { Establish working order } \\
\text { of equipment for } \\
\text { 2-stage operation; }\end{array}$ & $\begin{array}{l}8.3 \neq 1 \mathrm{~km} / \mathrm{s} \text { attained; } \\
\text { Armature dispersion } \\
\text { postulated to interpret } \\
\text { data; } \\
\text { Secondary arcs observed; } \\
\text { Velocity limited by the } \\
\text { length of the launcher; } \\
\text { Extrapolation of observed } \\
\text { performance to } 24 \mathrm{ft} \text { and } \\
3 \text { stages is expected to } \\
\text { produce } 12 \mathrm{~km} / \mathrm{s}\end{array}$ \\
\hline
\end{tabular}

components were completely dissociated into their atomic constituents. Typically, the package is positioned at about $2.5 \mathrm{~cm}$ behind the projectile with its cylindrical axis transverse to the axis of the bore. And the breech is strongly sealed with the breech plug about $2.5 \mathrm{~cm}$ behind the plasma generation package. The purpose is to use the pinching force of the initial current on the transverse cylindral foil to create a significantly high pressure to accelerate the combustion of the propellant. The initial plasma is then al lowed to expand rapidly to fill the volume between the projectile and the breech plug, so that as 
uniform a plasma as possible would be generated initially. The technique was demonstrated to be a success, leading to significantly reduced ablation of the rails, though ablation of the insulator wall remains severe.

\subsubsection{Group B}

This was followed by a second series of experiments (Group B), in which several projectile designs were tried for best performance in terms of plasma sealing and gauging of the walls. It was found that projectile compliance with the bore is an important factor. The use of a hemispherical cavity at the back of the projectile provides the neccessary compliance for plasma sealing, while the introduction of suitable fins (or groves) in the front portion of the projectile reduces the friction between the projectile and the walls and in turns reduces the occurrence of hypervelocity gouging.

\subsubsection{Group C}

Preliminary study of launcher (armature) behavior began with Group $C$ of the experiments. This series attempts to characterise the performance of the augmented launcher in relation to the effects of ablation and viscous drag. Through the combined use of the lithium propellant armature, the refractory rails (molydenum), and a lower current made possible by augmentation, ablation rates in these experiments were sufficiently low that its effects on the launch dynamics were so small that they could not be detected within the resolution of the diagnostics used. Consequently, the experimentally measured position-time profile from these experiments were easily simulated without the introduction of a time-varying arc mass by ablation (Figure 5.5). Using only $207 \mathrm{~kJ}$ of energy, with peak current below $250 \mathrm{kA}$ and less than $1.5 \mathrm{~m}$ of acceleration, a velocity of $5.3 \mathrm{~km} / \mathrm{s}$ was achieved with a $1.429 \mathrm{~g}$ polycarbonate projectile in these series of experiments (Shot DX1-103184-1). At that time this velocity represented one of the highest reproducible velocities reached in railguns in recent 


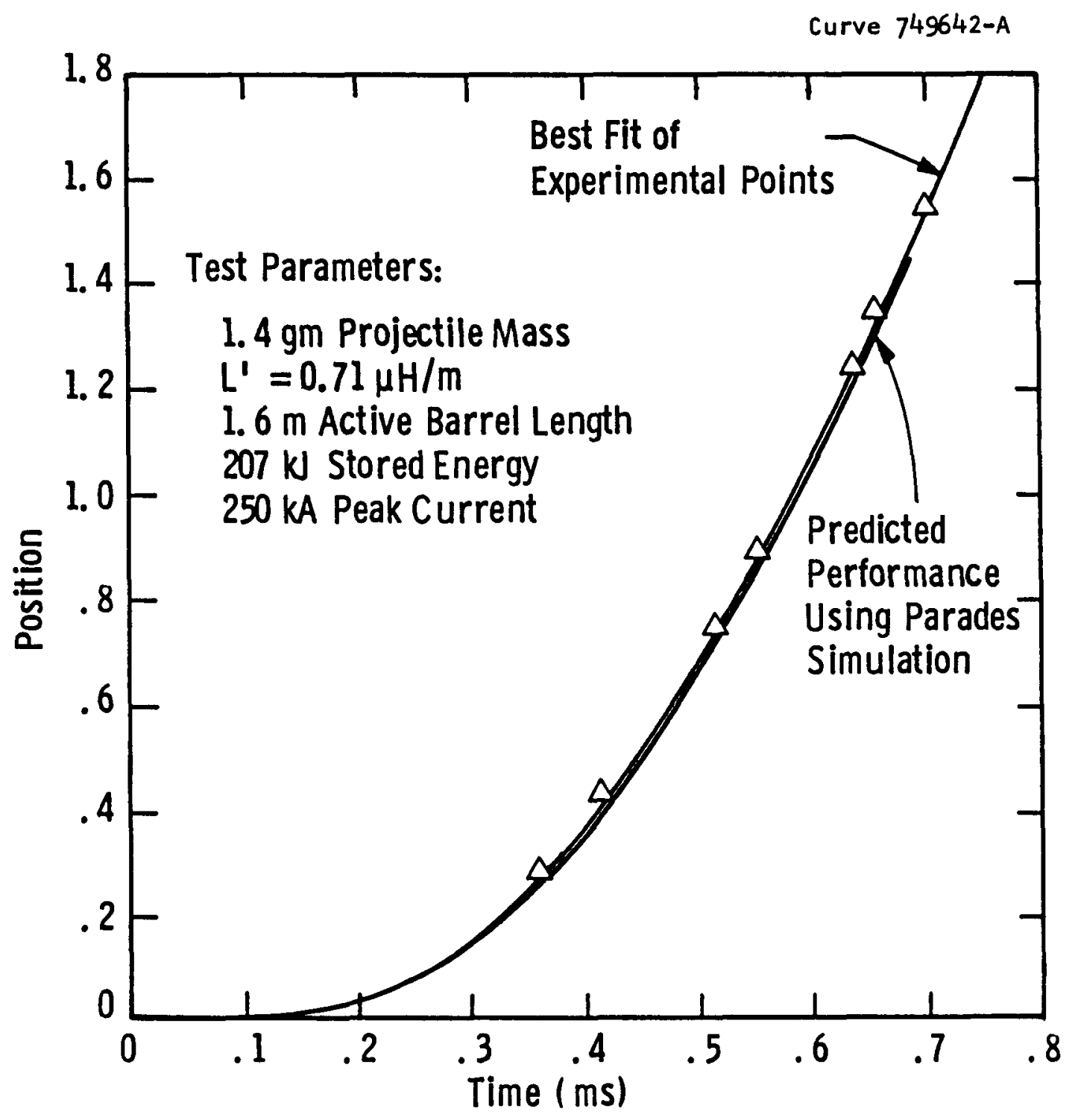

Figure 5.5 - Predicted and observed position profile obtained with SUVAC augmented first stage. 
years. The plasma generation package used in this shot consisted of a mixture of $102.9 \mathrm{mg}$ of nitrocellulose and $171.4 \mathrm{mg}$ of lithium nitrate wrapped into a cylindrical form in an aluminum foil weighing $36.4 \mathrm{mg}$. The bore was gauged to have the dimensions $9.43 \mathrm{~mm} \times 9.45 \mathrm{~mm}$. The measured dimensions of the projectile before being loaded into the barrel were $9.45 \mathrm{~mm} \times 9.47 \mathrm{~mm} \times 15.2 \mathrm{~mm}$. The friction force in loading the projectile was estimated to be approximately $89 \mathrm{kN}$.

Throughout most of the experiments of Group A, B, and $C$, a single pair of molybdenum rails were used, without the need of re-machining. Shots were repeated after a routine cleaning of the bore of the gun with acetone to remove any contamination of the insulator which might be conducting. Visually, the amount of erosion of the rails was greatly reduced when compared with the case in which the plasma was initiated with only an aluminum foil of about the same mass as that which is used to make the container for our plasma generation package. Figures $5.6,5.7,5.8$, and 5.9 show its conditions after the completion of these 3 series of experiments in which currents ranging from $100 \mathrm{kA}$ to $250 \mathrm{kA}$ were used. The plasma generation package was typically positioned at about 7.5 inches from the breech end of the rails. The indentations which can be seen in Figure 5.7 were machined into the rails to facilitate vertical loading of the projectile into the region between the rails. This system of loading the projectile was not used in later versions of the barrel due to a modification of the breech adaptor to provide better support of the rails in this region.

\subsubsection{Group D}

Next we conducted a series of experiments in order to check our theoretical expectations of the relative performance between an augmented launcher and an un-augmented launcher. The experiments were conducted using a section of SUVAC-II barrel operated in a nonaugmented mode. The pulse forming inductance of the circuit was reduced so that a higher peak current could be produced from the same amount of 


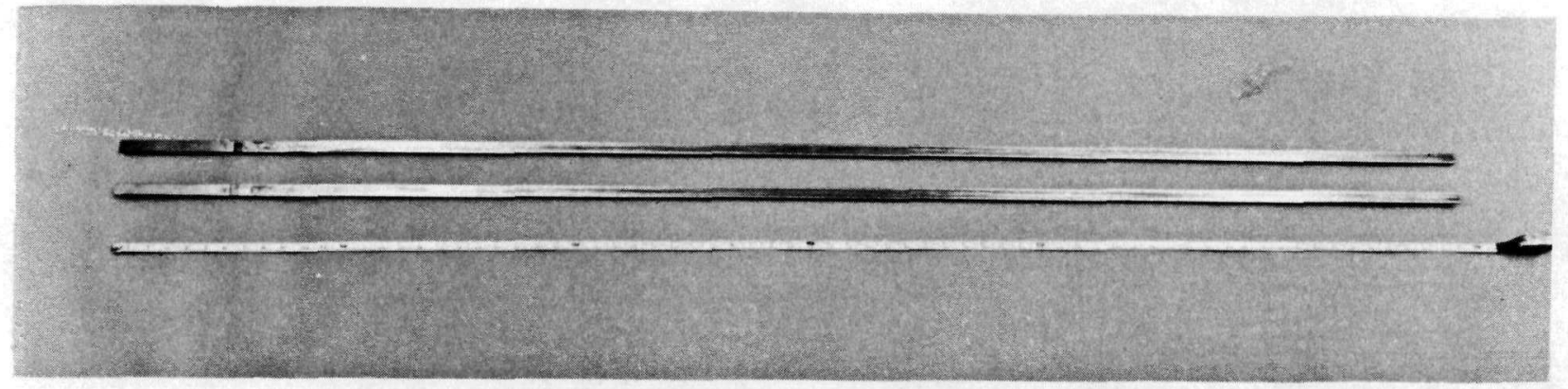

Figure 5.6 - Molybdenum rails used in SUVAC-I experiments: The rails have been tested in more than 20 shots with currents in the range from $100 \mathrm{kA}$ to $270 \mathrm{kA}$. The total rail width is $15.88 \mathrm{~mm}$; the part exposed to the plasma is nominally $9.5 \mathrm{~mm}$ wide (with bore width). 


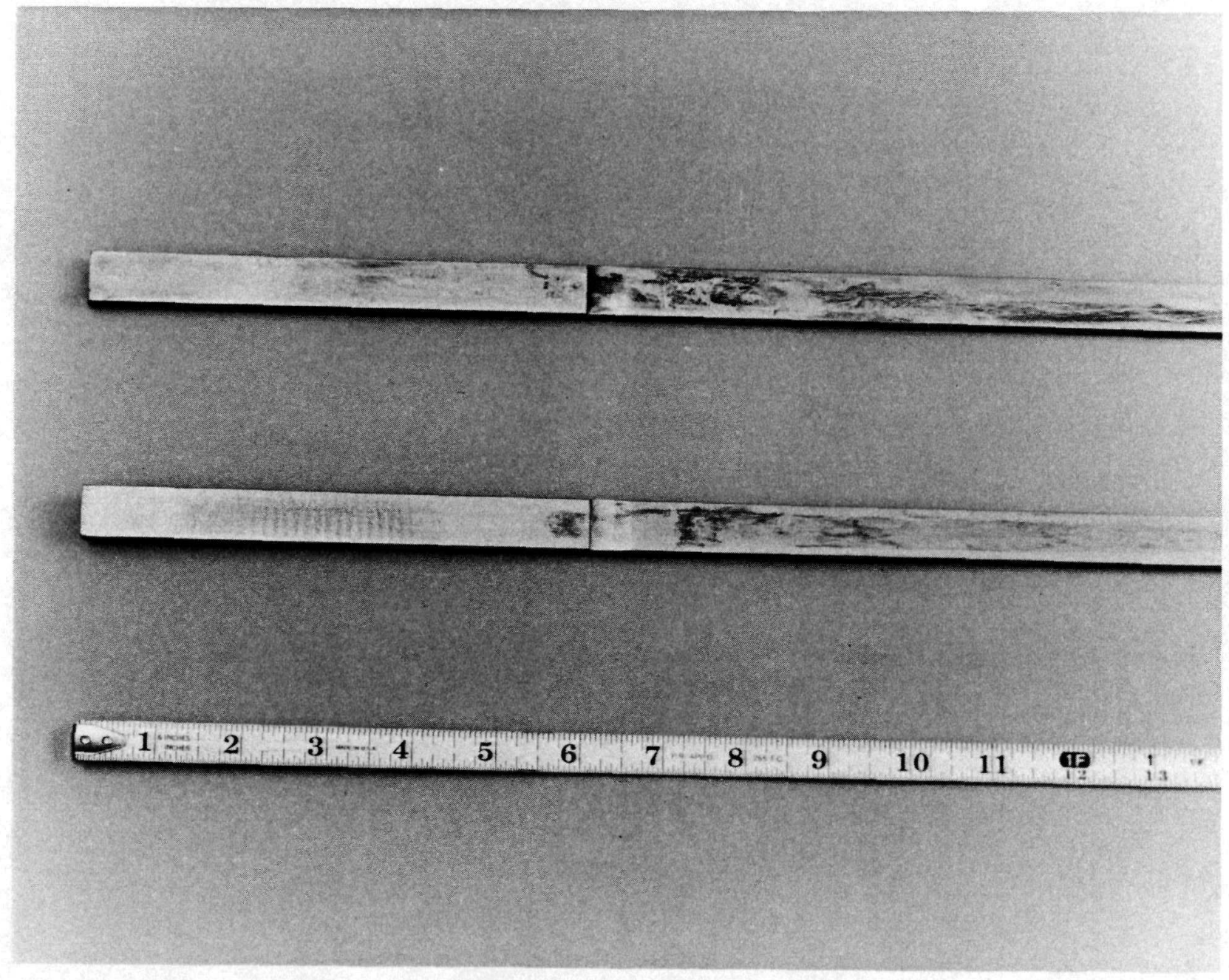

Figure 5.7 - SUVAC-I Test Series: Showing the first 6 inches of the molybdenum alloy rails from the plasma initiation point. The plasma generation package was positioned typically at 7.5 inches from the breech end of the rails. 


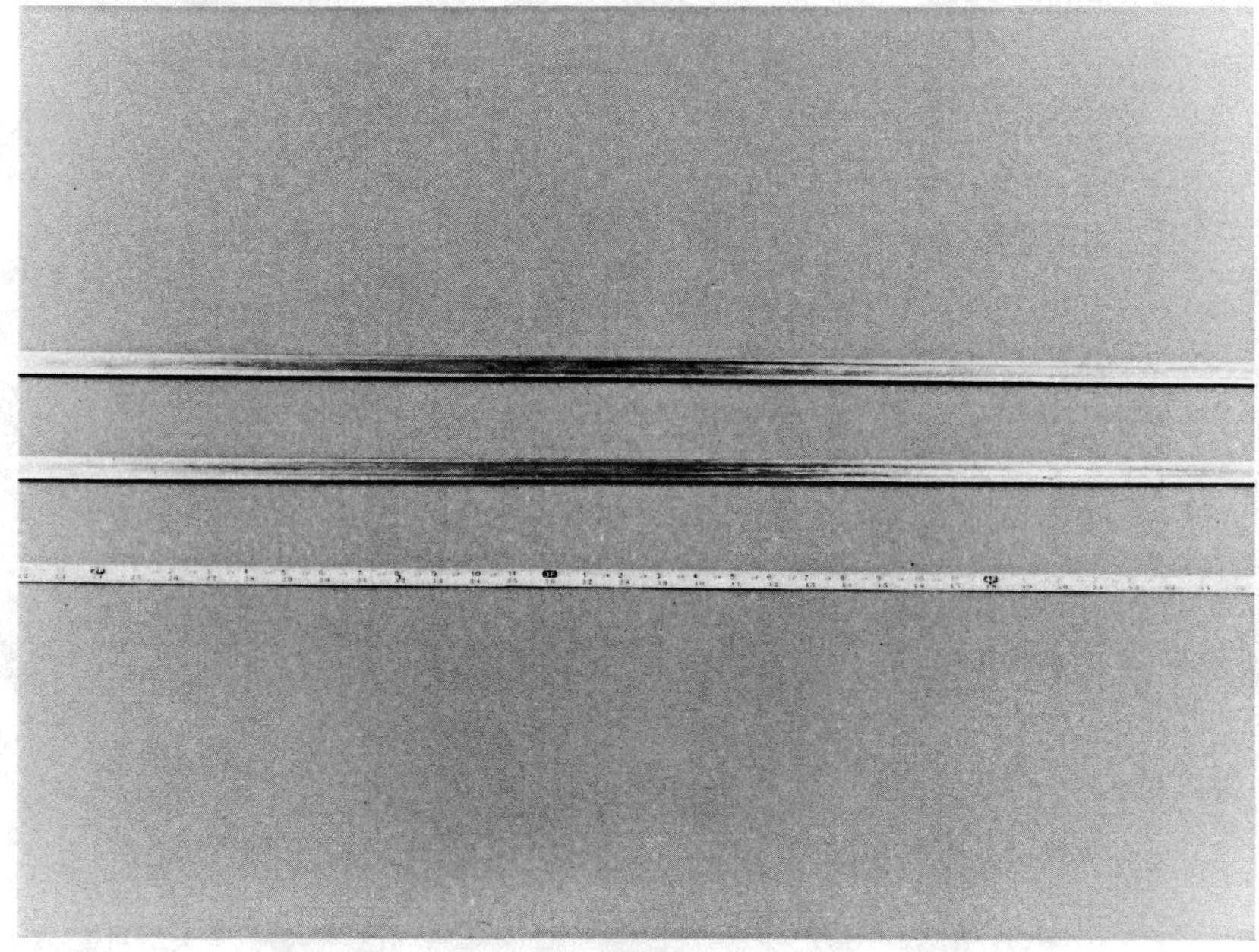

Figure 5.8 - SUVAC-I Test Series: Showing the middle portions of the rails used in the SUVAC-I augmented first stage experiments. 


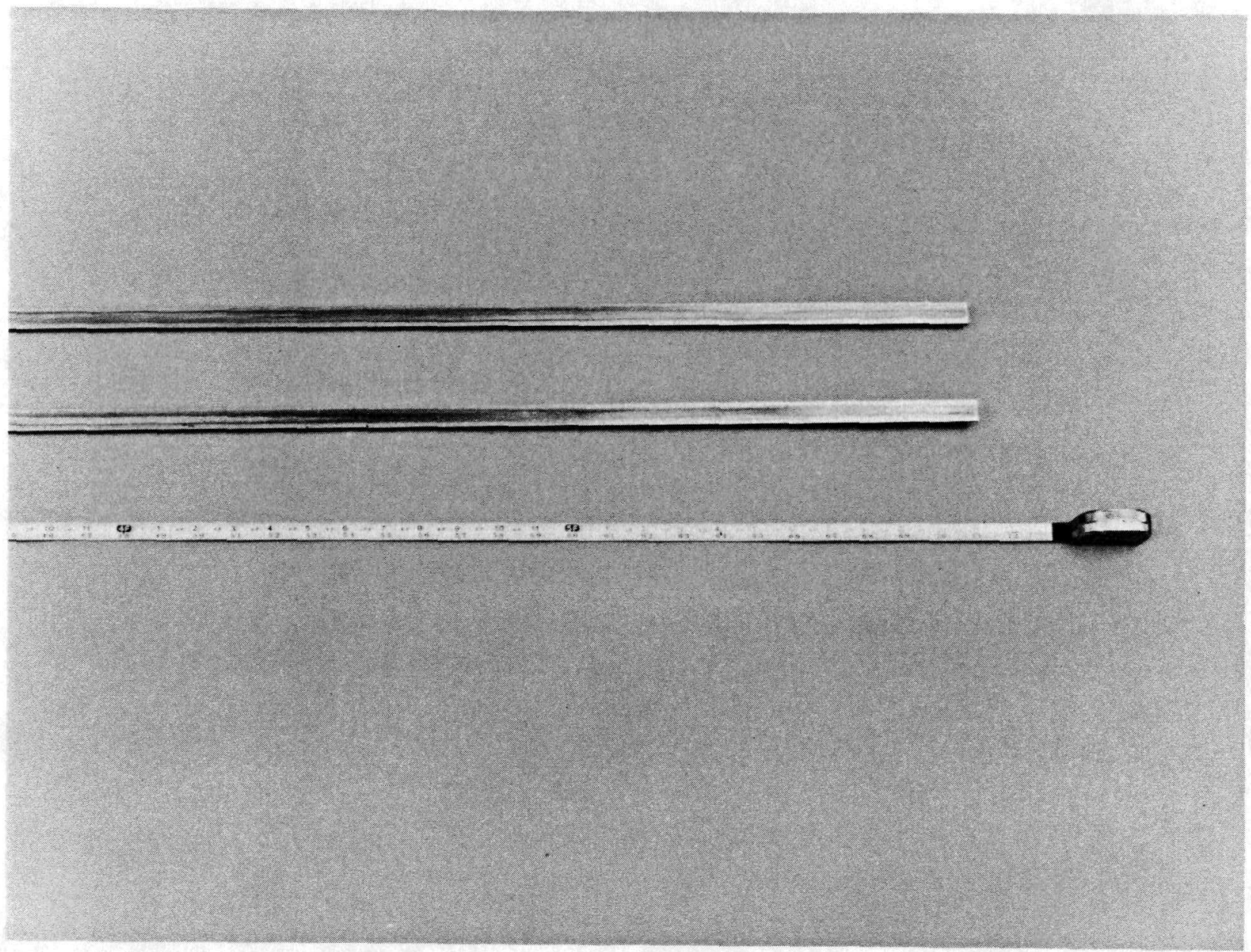

Figure 5.9 - SUVAC-I Test Series: Showing the muzzle end of the rails used in the SUVAC-I augmented first stage experiments. 
initial energy in the capacitor bank. This higher current is required in order to offset the lower inductance gradient in the non-augmented barrel so that the same Lorentz force on the armature is produced. We found that, with equivalent stored energy from the capacitor bank and equivalent peak Lorentz force produced by the current, the non-augmented launcher consistently produce a lower velocity or lower kinetic energy in the projectile than the augmented launcher.

With the same amount of energy of $207 \mathrm{~kJ}$ in the power supply, and a considerably higher peak current (390 kA) and a smaller projectile mass ( $1.15 \mathrm{~g}$ ) than the experiment (Shot DX1-103184-1) cited above, the highest velocity reached in this group of experiments was $4.5 \mathrm{~km} / \mathrm{s}$ (Figure 5.10(a), Shot CX2-120584-1). The final kinetic energy of the projectile was only $60 \%$ of that obtained in equivalent Shot DX1-103184-1 with an augmented launcher. In this shot, the polycarbonate projectile measured $9.45 \mathrm{~mm} \times 9.6 \mathrm{~mm} \times 13.26 \mathrm{~mm}$, and the loading friction force was approximately $100 \mathrm{kN}$. The plasma generation package consisted of a mixture of $162 \mathrm{mg}$ of nitrocellulose, $170 \mathrm{mg}$ of lithium nitrate contained in an aluminum foil cylinder of $41 \mathrm{mg}$. Four of the magnetic probe signals from this shot are shown in Figure 5.10 (b). In this shot, the occurrence of a secondary arc was first noted as can be seen from the second peak in the $\mathrm{dB} / \mathrm{dt}$ signals of Figure 5.10 (b).

Significantly higher ablation rate per unit Lorentz force was observed in this series of experiments. Consequently the dynamical effects of ablation on the interior ballistics of the projectile is clearly resolvable within the accuracy of the diagnostics. This series of experiments provided an opportunity to estimate the arc mass and the corresponding ablation rate measured in $g$ per $M J$ of energy dissipated in the arc. Without augmentation, it is also easier to obtain an estimate of the power dissipation in the arc from the measured muzzle voltage. We note with Jamieson that the muzzle voltage gives only the voltage 


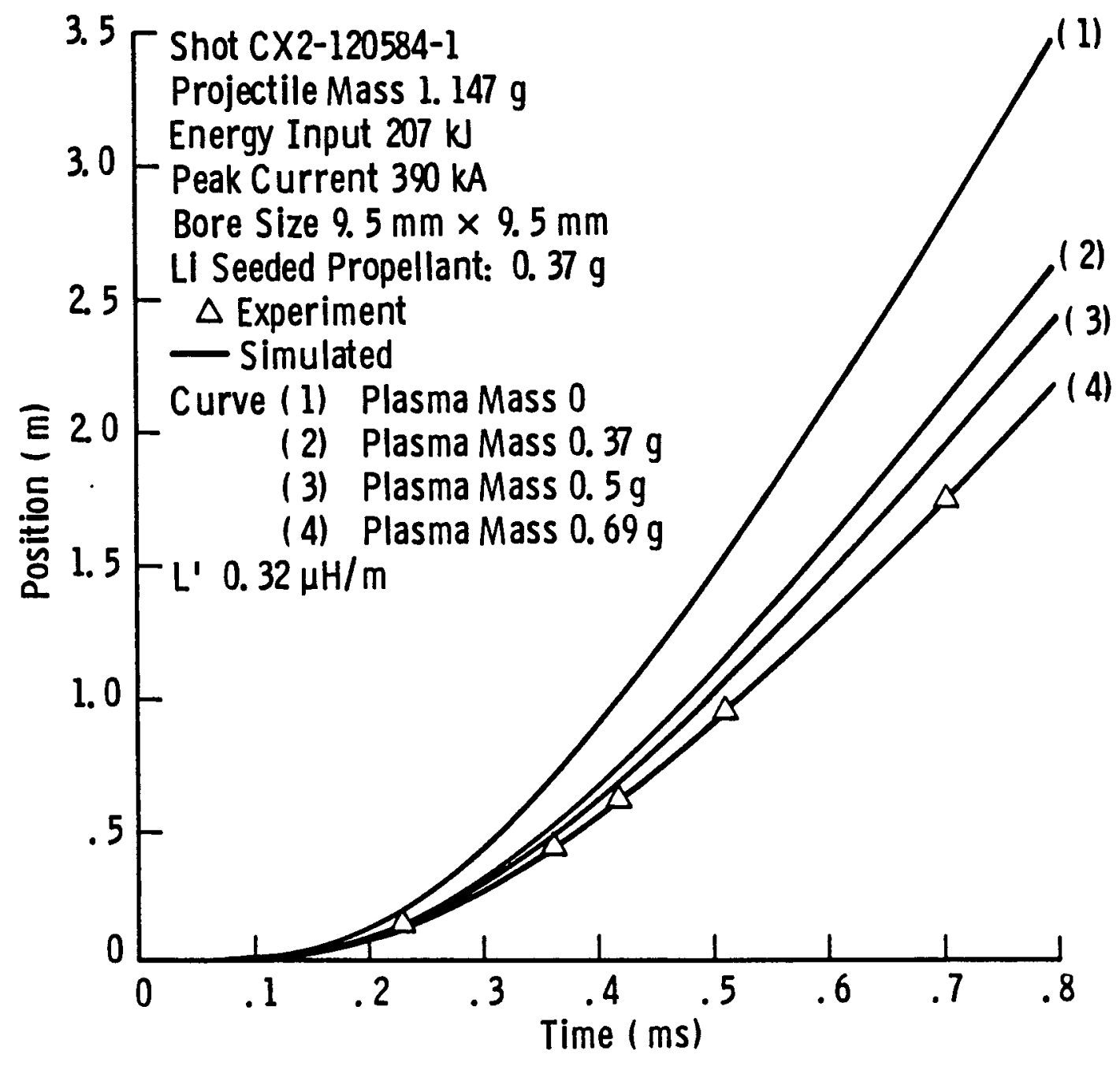

Figure 5.10(a) - Observed and simulated position profile in Shot CX2-120584-I with SUVAC-II un-augmented barrel in a single stage configuration: The experimental points are deduced from the magnetic and optical probes. The 4 simulated curves shown correspond to 4 different values used for the mass of the plasma armature. Curve (2) uses the mass of the initial plasma generation package. Curve (4) uses a plasma armature mass which provides the best fit with the experimental points. 

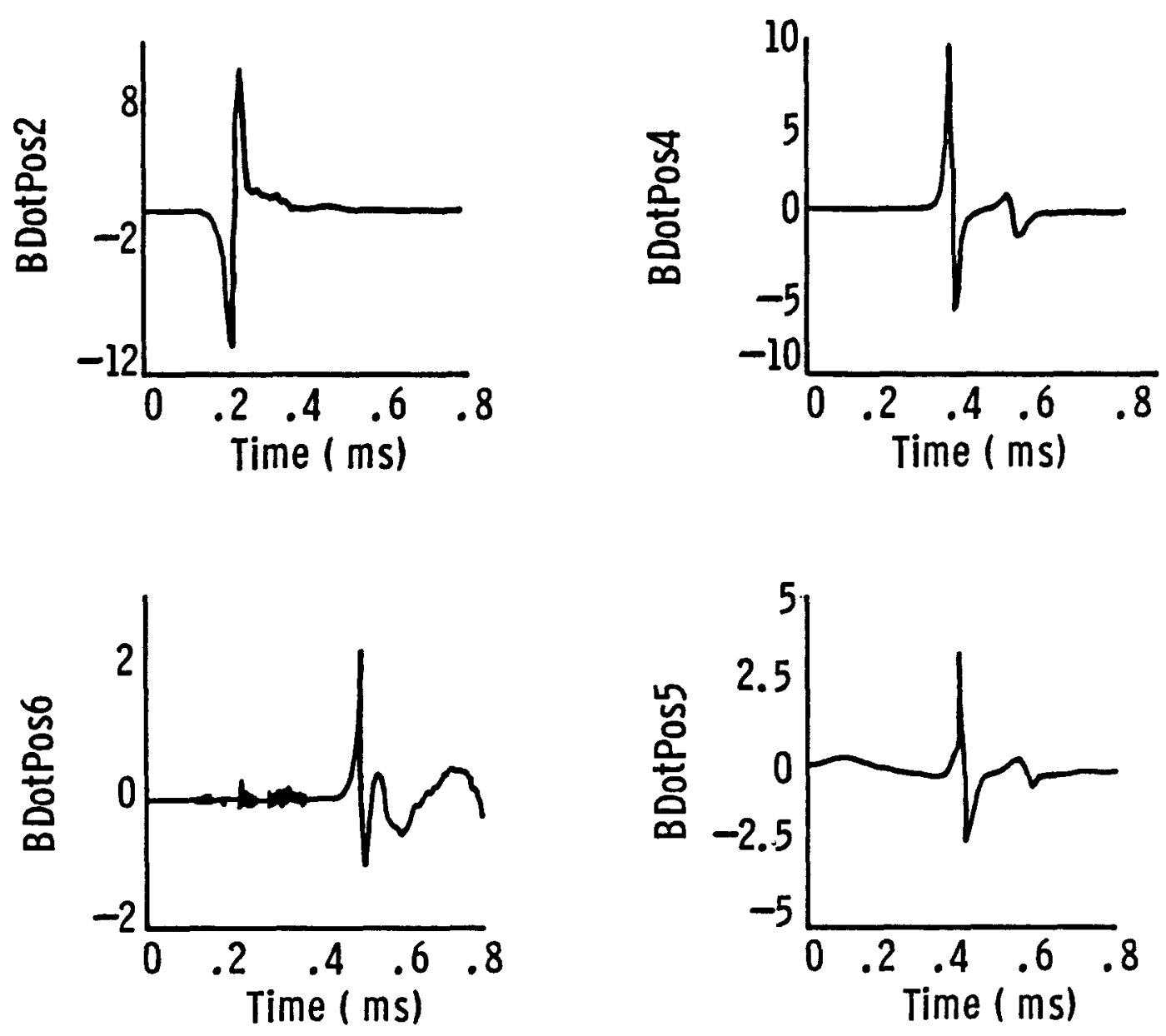

Figure $5.10(\mathrm{~b})$ - Voltage signals from the magnetic pick-up loops. The instantaneous voltage value is a measure of the time rate of change of the horizontal component of the magnetic field produced at the loop by the moving armature current. Note the appearance of a secondary signal following the primary signal indicating the occurrence of a secondary arc which may have been created by restrike or break-up of the primary one. The secondary arc seems to nearly catch up with the primary arc at the probe BDOTPOS6. 
drop across the leading edge of the plasma armature, and does not neccessarily represent the ohmic drop across the whole of the armature. Experimentally, however, the muzzle voltage is the only voltage measurement we have which is most related to the armature ohmic drop, and thus we use it as our best estimate of the ohmic drop across the arc. Better measurement of the ohmic drop across of the arc is required in the future. From the estimated arc mass and the estimated ohmic dissipation in the arc in this series of experiments, the effective ablation coefficient were estimated to have values ranging from $6 \mathrm{~g} / \mathrm{MJ}$ to $15 \mathrm{~g} / \mathrm{MJ}$.

For Group D experiments, we often found it possible to fit the experimentally measured position-time profile with a simulation using only a fixed mass for the arc, as shown in Figure 5.10. This procedure was not found possible by Parker in the experiments he described[9], in which he found that a true time-varying arc mass is neccessary to simulate his experimental results computationally. The likely explanation for this difference is as follows. In our experiments, the plasma armature is initiated with a considerably larger number of particles. But more particles are needed to to stop radiative ablation. These are supplemented from ablating the walls. Because of the larger number of particles initially, this ablation needs to occur for only a shorter time, most likely to be in a short interval centering about the instant at which the current peaks (0.2 ms in Shot CX2-120584-1) in the early phase of the acceleration. After this period, the armature attains a sufficiently low temperature so that radiative ablation is reduced to an insignificant level. In this later phase, the armature mass remains almost constant.

In contrast, if the plasma were to be initiated with a much smaller number of particles, radiative ablation would continue for a much longer period, possibly extending into the late phase of the 
acceleration. When that occurs, the ablation effect on the launch dynamics would be further amplified by the higher velocity in this later phase. Consequently, the position-time profiles in such experiments can only simulated properly using a dynamic arc mass throughout most of the launch period. This later circumstance is probably what happens in the experiments described by Parker.

\subsubsection{Group E}

The experiment groups $A, B, C$, and D were all conducted on the SUVAC-I launcher system using a 6-ft barrel. On completion of these series, the SUVAC facility was upgraded. Besides the SUVAC-II launcher, the upgrade includes the commissioning of an entirely new data acquisition system, a new control system, and a new firing system for multi-stage operation.

The new data acquisition system, shown in Figure 5.11 , is built around the CAMAC architecture supported by an GPIB bus for communication between each CAMAC crate and the system controller. The system controller is an IBM AT microcomputer equipped with a National Instrument GPIB board, so that the IBM AT actually functions as a GPIB bus controller, and each CAMAC crate appears to the bus as an GPIB instrument. The CAMAC crate talks and listens to the bus through a CAMAC-GPIB interface module (LeCroy Module 8901) which resides on slot 24 and 25 of the CAMAC crate. The digitizers used are standardized on LeCroy Model TR8837 transient digitizers, each of which provides a channel of recording at a sampling rate of 32 million data samples per second, and a recording length of 8192 8-bit samples per sweep. Altogether, the new data acquisition system has 50 of these high-speed long-record digitizing channels.

The new control system capable of controlling multi-stage operation of the power supply is shown in Figure 5.12. 


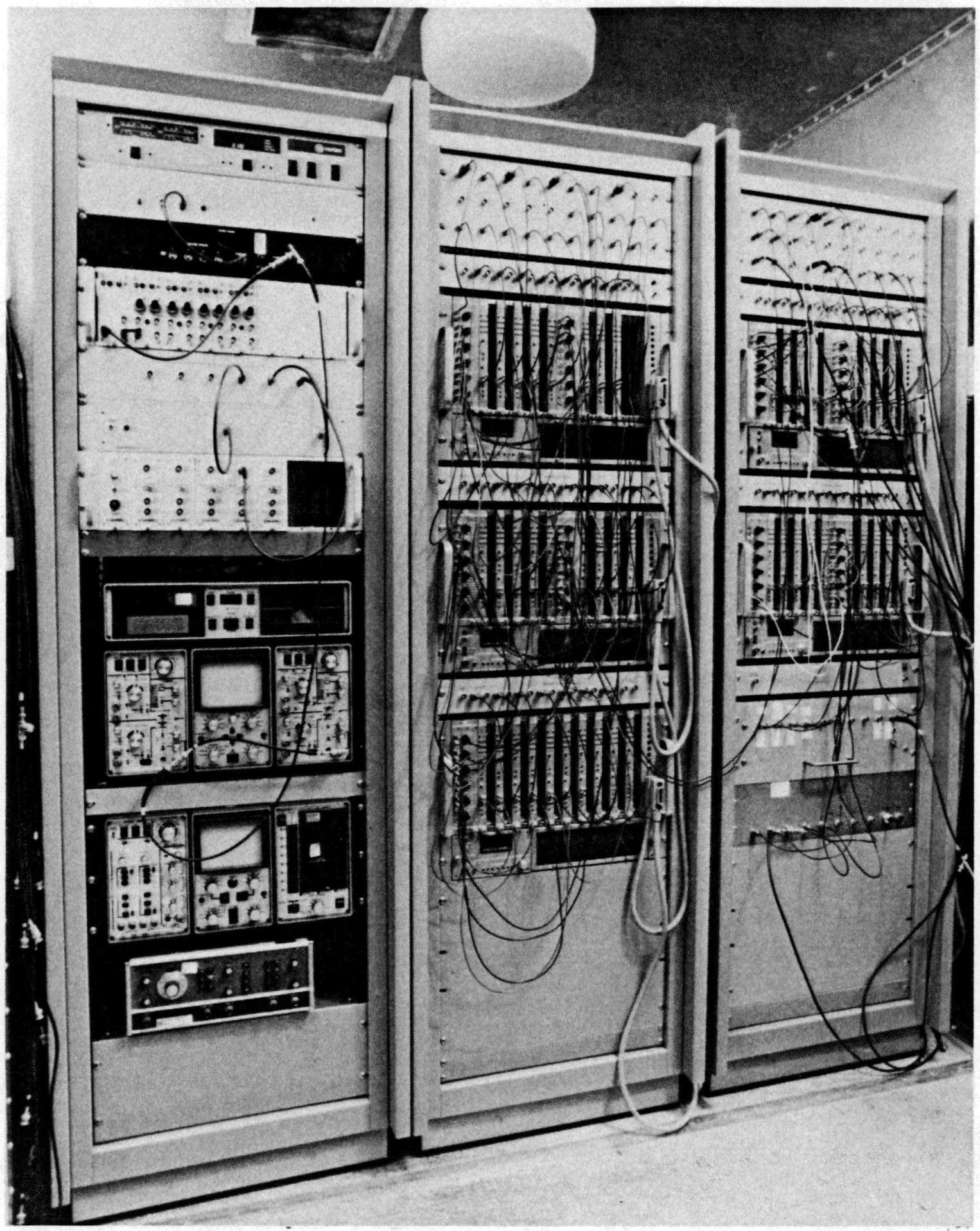

Figure 5.11 - Data acquisition system. 


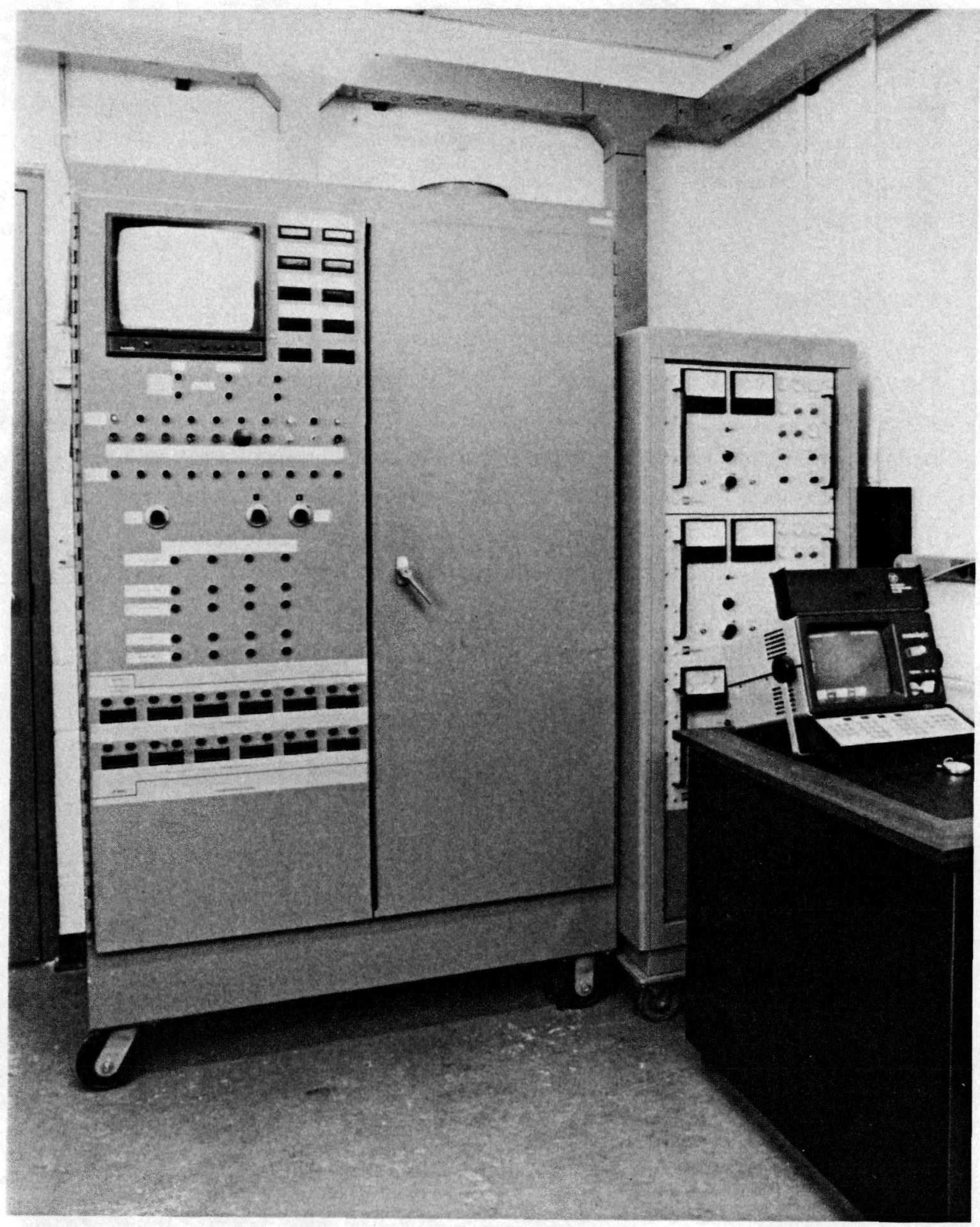

Figure 5.12 - Control system. 
The SUVAC-II launcher system comprising the 1-MJ power supply, the 12-ft barrel, the two independent high voltage chargers, the data acquisition system and the control system were built and installed in the period between April 1985 and August 1985. The system was integrated and commissioned in September 1985.

\subsubsection{Group F}

Systematic investigations of the SUVAC-II 12-ft launcher with the 2-stage power supply began with this group of experiments. The experiments range from operating the 12-ft launcher in a single-stage mode, or 2-stage mode with current injection of both stages at the breech, or in a 2-stage mode with current injection at the breech from stage 1 power supply and at the mid-section of the launcher from stage 2 power supply. In the last mentioned mode, SUVAC-II is a 2-stage DES launcher $[30,31,32]$. A total of 14 experiments were conducted.

Velocities ranging from $4 \mathrm{~km} / \mathrm{s}$ to $8 \mathrm{~km} / \mathrm{s}$ were recorded by the magnetic and optical sensors in these experiments.

The highest velocity with the most complete set of data in this series was obtained in the experiment OPEN-X1-05. In this experiment, all of the critical behavior of the railgun launcher relevant to the attainment of ultrahigh velocity can be seen, including the instability and dispersion of the open plasma armature, the existence of a long plasma tail, the occurrence of secondary arcs, and the dynamical effects of ablation and viscous drag at high velocity.

Figure 5.13 shows a picture of the experimental set up used, a schematic of which is shown in Figure 5.14. Stage 1 power supply consists of a $8.2 \mathrm{mF}$ capacitor bank charged to a voltage of $6.04 \mathrm{kV}$ giving it a stored energy of $150 \mathrm{~kJ}$, whereas stage 2 has $11.88 \mathrm{mF}$ charged to $4.74 \mathrm{kV}$ giving it $133 \mathrm{~kJ}$ of energy. Both sections of the launcher are augmented, with mutual inductance gradient of $0.17 \mu \mathrm{H} / \mathrm{m}$ and 


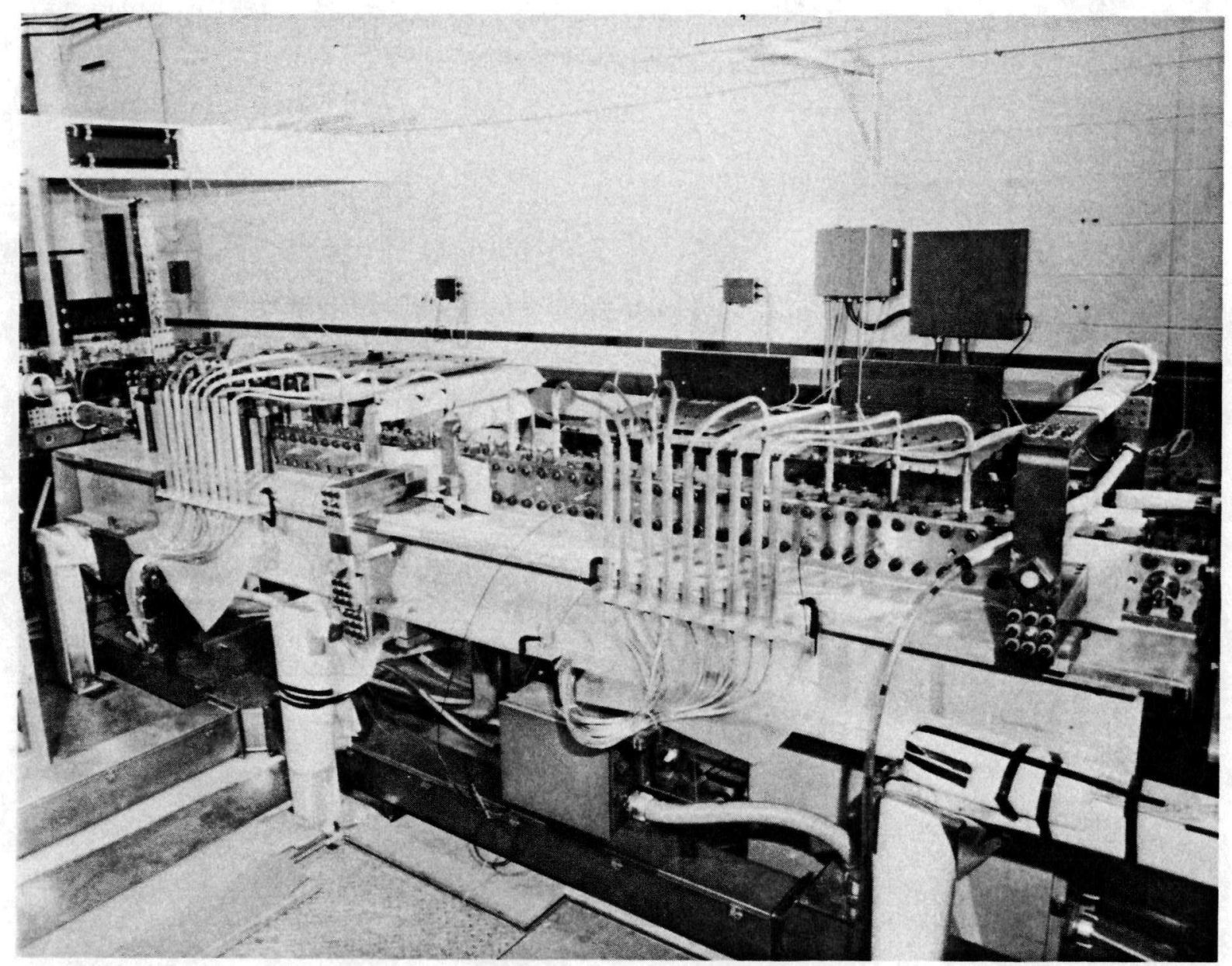

Figure 5.13 - The 12-ft SUVAC-II launcher configured as a 2-stage DES accelerator with current injection at mid-section. 
Dwg. 9383A71

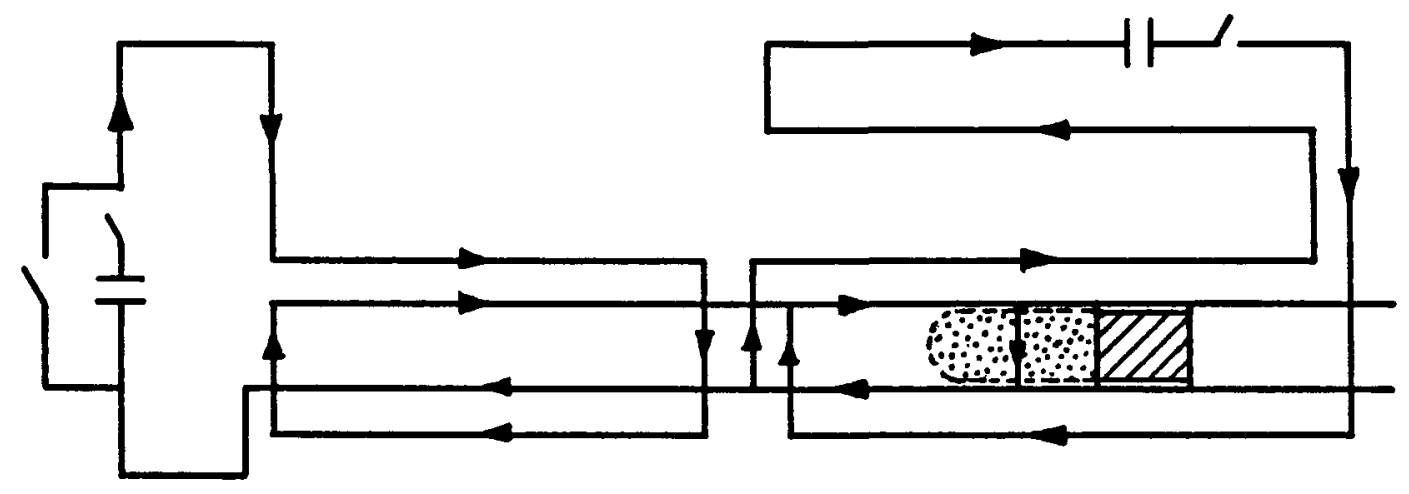

Bore $\quad 9.09 \mathrm{~mm} \times 9.80 \mathrm{~mm}$

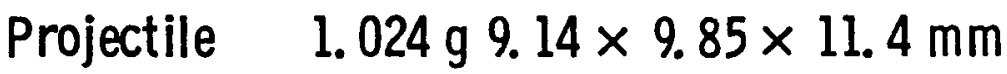

$\begin{array}{lcccc} & \begin{array}{c}L^{\prime} \\ \mathrm{uH} / \mathrm{m}\end{array} & \begin{array}{c}\mathrm{M}^{\prime} \\ \mathrm{uH} / \mathrm{m}\end{array} & \begin{array}{c}\text { Energy } \\ \mathrm{kJ}\end{array} & \begin{array}{c}\text { Current } \\ \mathrm{KA}\end{array} \\ \text { Stage 1 } & 0.34 & 0.17 & 150 & 198 \\ \text { Stage 2 } & 0.32 & 0.16 & 133 & 280\end{array}$

Figure 5.14 - System configuration and parameters for shot OPEN-X1-05. 
inner rails self inductance gradient of $0.32 \mu \mathrm{H} / \mathrm{m}$. Current injection from Stage 2 power supply is at the mid-section of the launcher, and was turned on with a pre-set delay of $509 \mu \mathrm{s}$ from the turn-on of the first stage. Figure 5.15 shows the current profiles obtained from a time integration of the Rogowski coil signals. The figure shows the current feeding the breech (Stage 1) and the junction (Stage 2) as well as the total current in the bore of the gun at any time; this must not be construed to be the total current at any particular location of the launcher $[30,31,32]$. Current produced by Stage 1 power supply reaches a maximum of $198 \mathrm{kA}$ at $343 \mu \mathrm{s}$, while Stage 2 power supply produces a peak of $279 \mathrm{kA}$ at $852 \mathrm{ks}$. The total current in the bore reaches a peak of 418 $\mathrm{kA}$ at $827 \mu \mathrm{s}$.

A sketch of the projectile used is shown in Figure 5.16. The projectile weighs $1.02 \mathrm{~g}$. The plasma generation package is made up of $50.3 \mathrm{mg}$ of nitrocellulose, $36.8 \mathrm{mg}$ of $\mathrm{LiNO}_{3}$, and $35.1 \mathrm{mg}$ of $\mathrm{LiH}$ placed in a cylindrical container made of $27.2 \mathrm{mg}$ of aluminum foil.

Figure 5.17 shows the witness plate showing the hole as punched out by the projectile.

The rails used consisted of a pair of copper rails which have been first cladded with a layer of tungsten to a thickness of $0.3 \mathrm{~mm}$, using Metco technique of plasma spray. It is then ground down to give a surface with controlled dimension and smoothness. The final thickness of the tungsten clad is $0.12 \mathrm{~mm}$. The rails had been used in 4 other shots with similar current level before this experiment and had been reused in these experiements with some polishing of the surface with a grinding stone in between shots. The condition of the rails after Shot OPEN-X1-05 is shown in Figure 5.18 for the first 6 inches immediately after the position of plasma initiation.

Figure 5.19 shows the collection of the traces of the magnetic probe signals. If the current is uniformly distributed in the arc, the 
Curve 749913-A

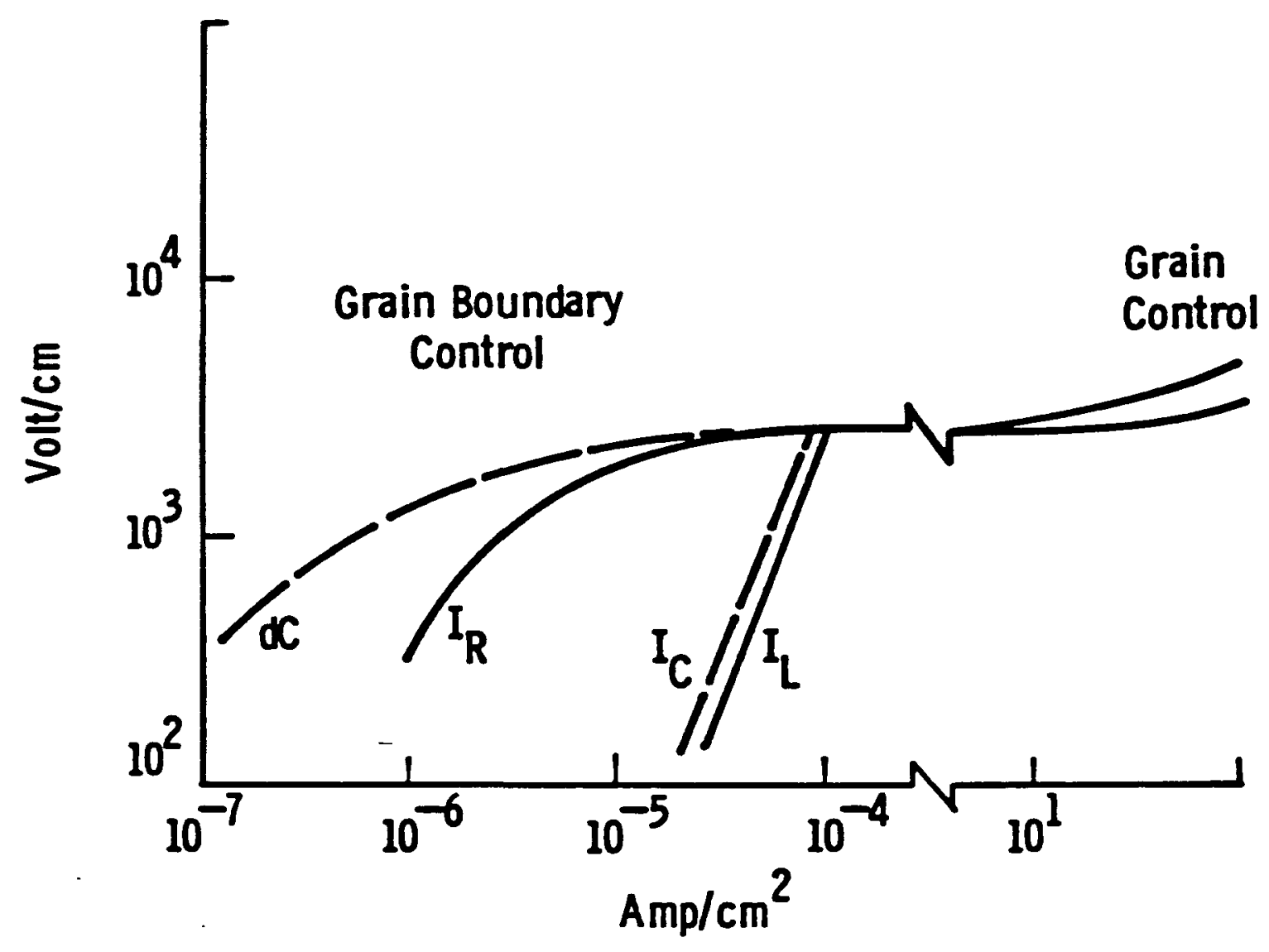

Figure 5.15 - Current profiles obtained from integration of Rogowski coil signals in Shot OPEN-X1-05. 
Dwg. 9385475 .
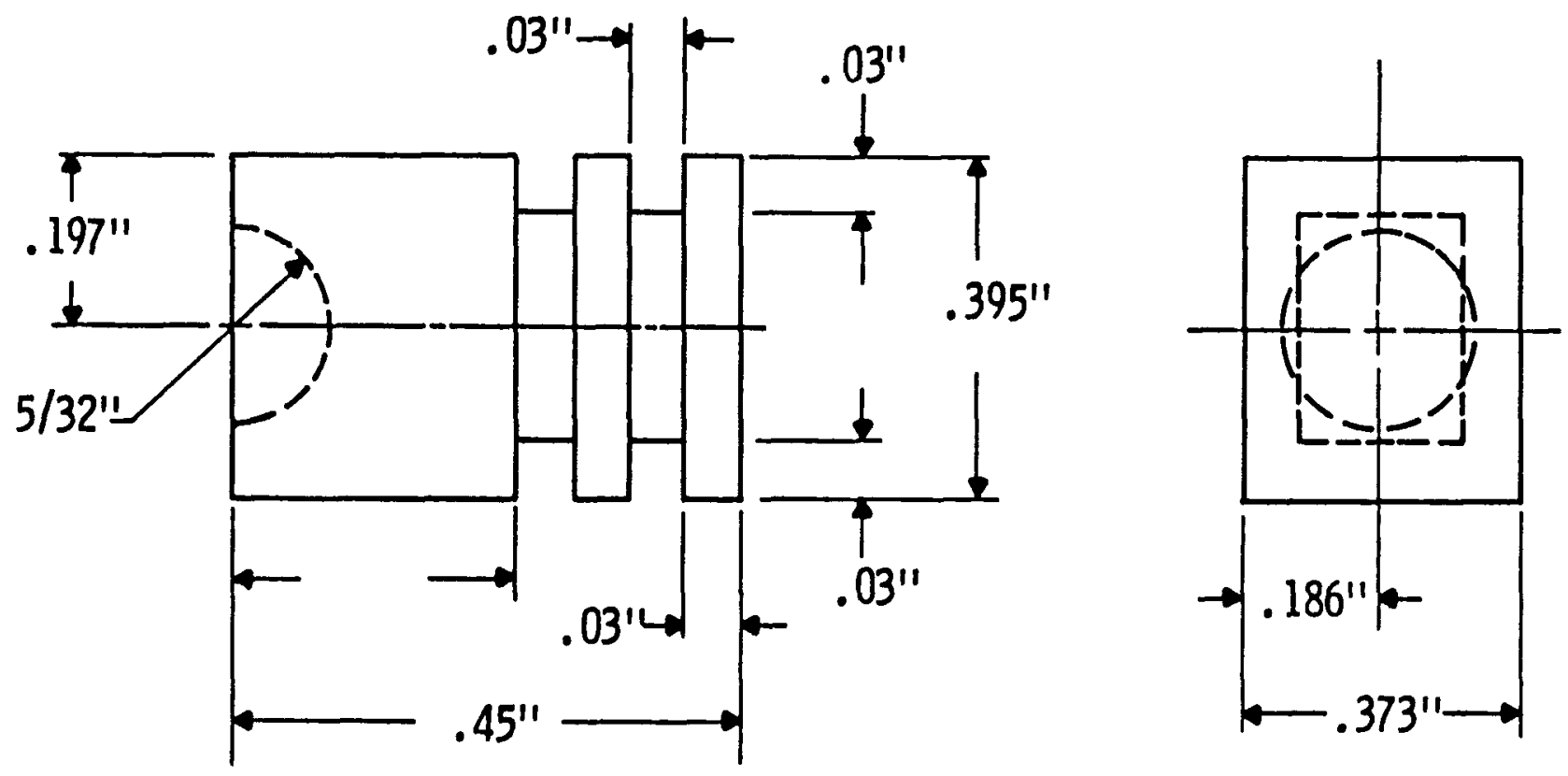

Figure 5.16 - A sketch of the projectile showing its dimensions as used in Shot OPEN-X1-05. 


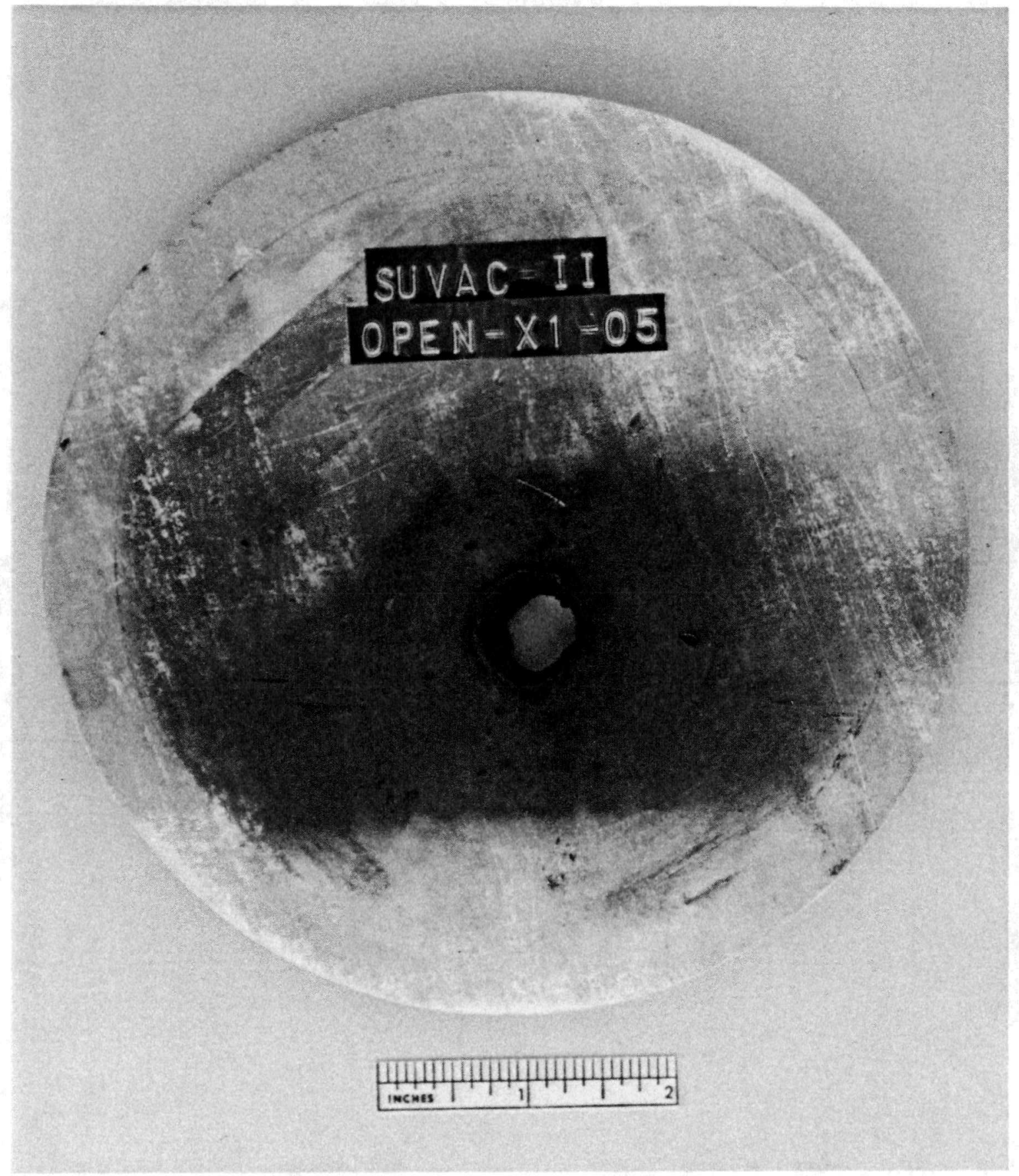

Figure 5.17 - The target plate used in Shot OPEN-X1-05 showing the hole punched out by the Lexan projectile. The target plate consists of a $1 / 4$ inch thick 6061 alloy aluminum plate. 


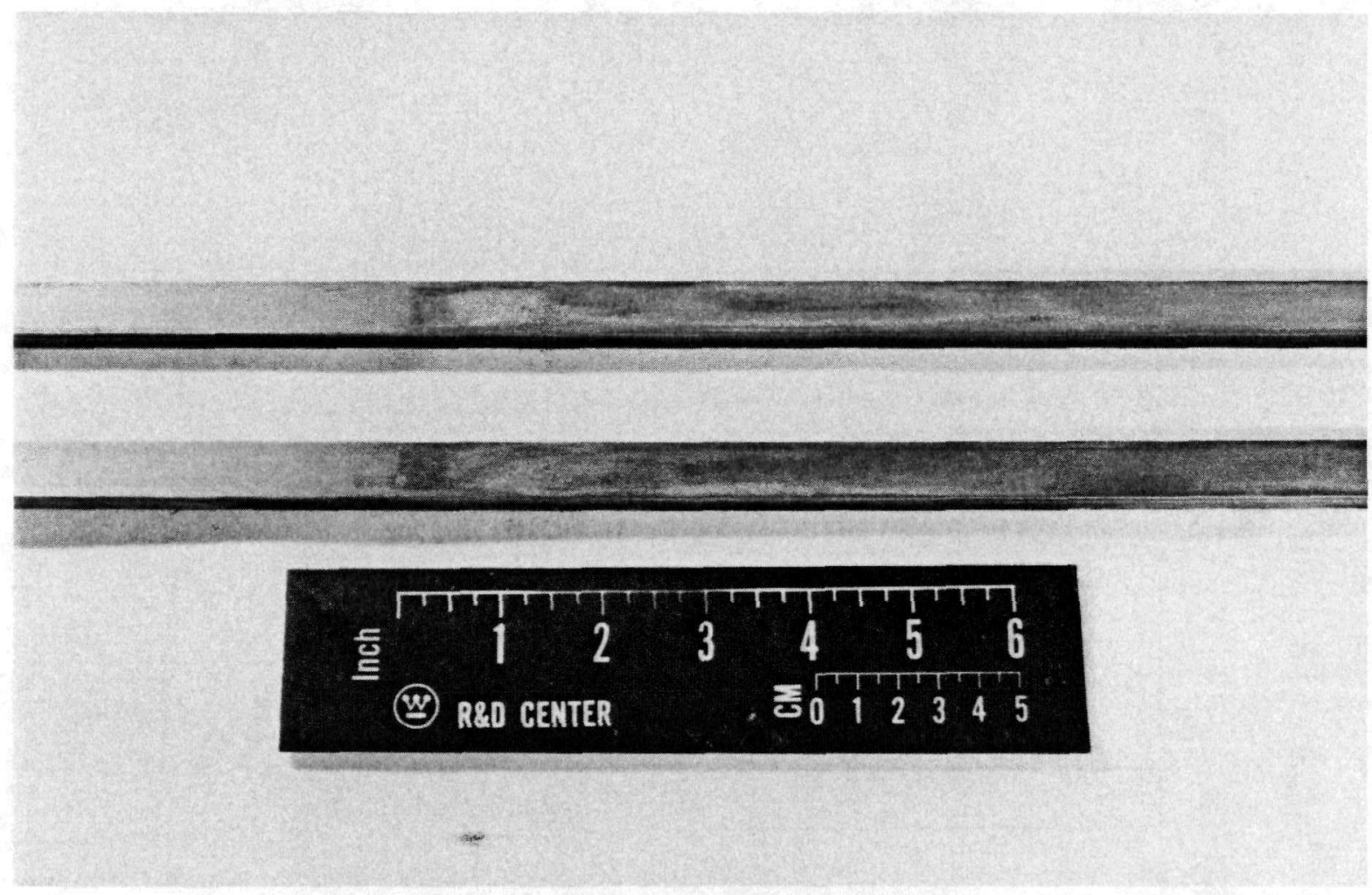

Figure 5.18 - The rails after Shot OPEN-X1-05. They had been used in four previous experiments with similar current from Stage 1. 
Curve 752334-A

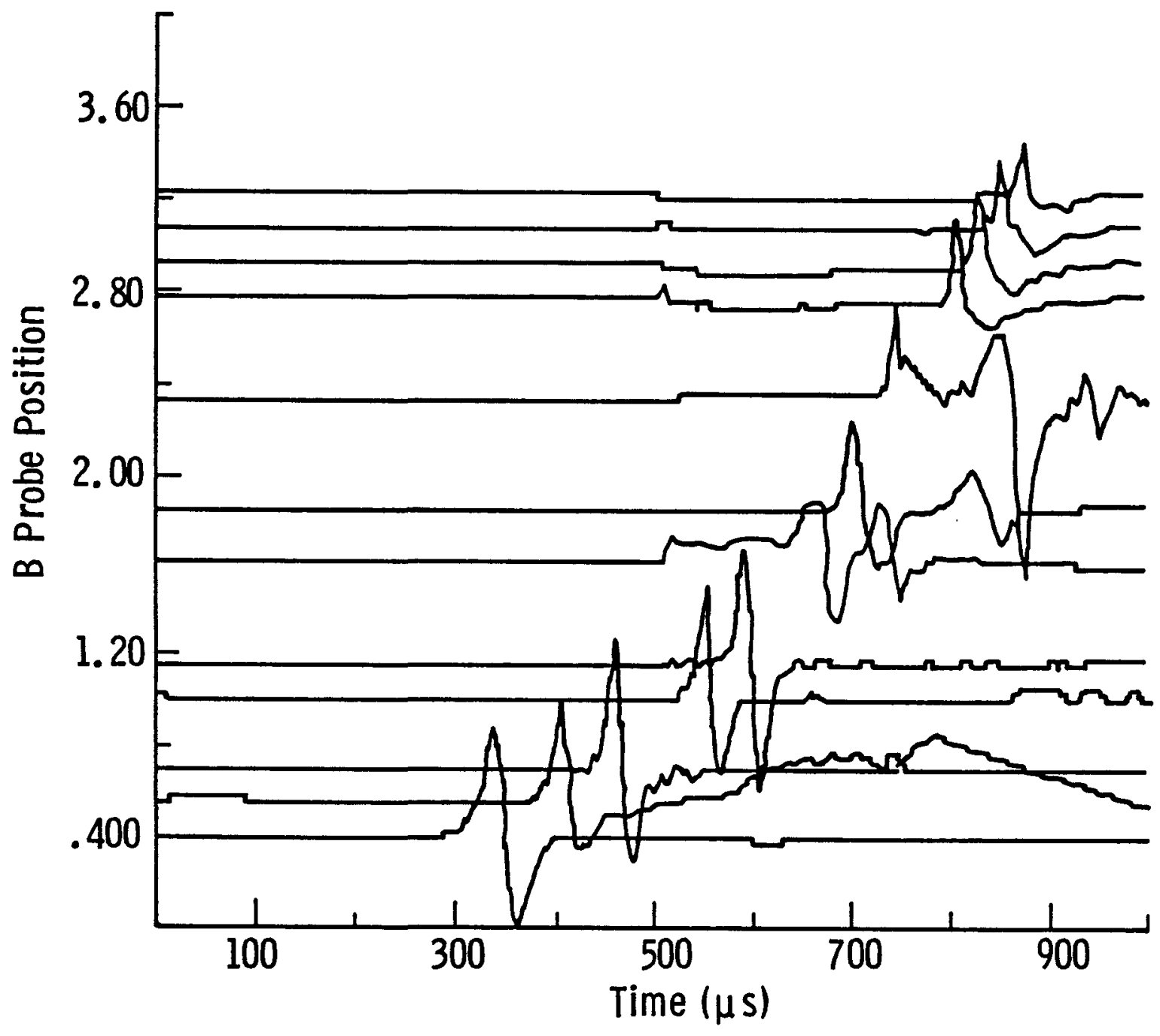

Figure 5.19 - Essemble of the magnetic probe signals from Shot OPEN-X1-05. 
first peak of the $d B / d t$ signal would correspond to the fly-by of the leading edge of the arc past a given probe. If the current density is not uniform but peaks towards the rear of the armature, then the leading edge of the arc could correspond to any point on the leading edge of the $d B / d t$ signal, between the foot and the peak of the signal. For interpreting this shot, for want of exact information about the current distribution in the arc, we take the average of the times for the peak, the midpoint of the leading edge and the foot of the signal as the time for the fly-by of the leading edge of the armature. Correlating these times with the known positions of these probes, we obtain a collection of points relating the positions of the plasma armature to time, which are shown as circles in Figure 5.20 .

In this experiment, we have also the benefit of 6 optical probes which are essentially brightness sensors in the visible part of the optical spectrum. These probes are inserted flush with the bore at 6 different locations of the barrel, and thus provide timing informations of the arrival of the leading edge of any arc at these locations. We have shown these as squares in Figure 5.20 .

By dividing the distance between two neighbouring probes by the difference in times of arrival at these probes, the average velocity of the armature (front) between these probes can be obtained. These mean velocities are shown in Figure 5.21. Again, the points represented by circles are obtained from the $\mathrm{dB} / \mathrm{dt}$ probes, whereas those derived from the optical probes are represented as squares.

With respect to Figure 5.19, two minor comments are in order: (1) We note that the slowly varying hump after the bipolar signal of the 2nd probe is due to an inadvertently poor magnetic field shielding of the signal cable; and (2) the signal cable for probe 4 was checked after the shot to behave capacitively and acted as a low-pass filter. This accounts for the very sharp rise from zero and quick plateauing to zero of the signal of this probe. 


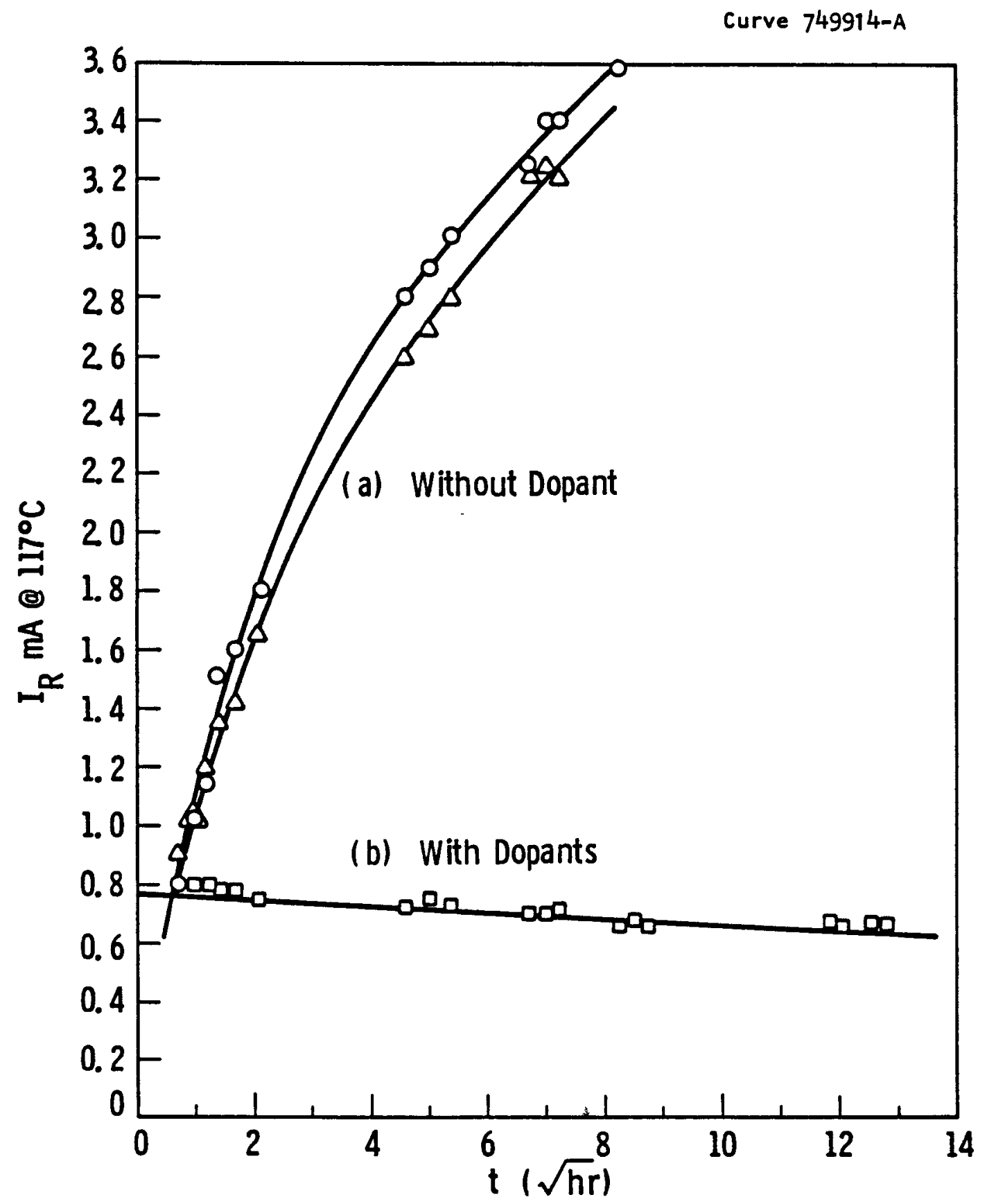

Figure 5.20 - Position-time history of the armature in Shot OPEN-X1-05. 
Curve 751915-A

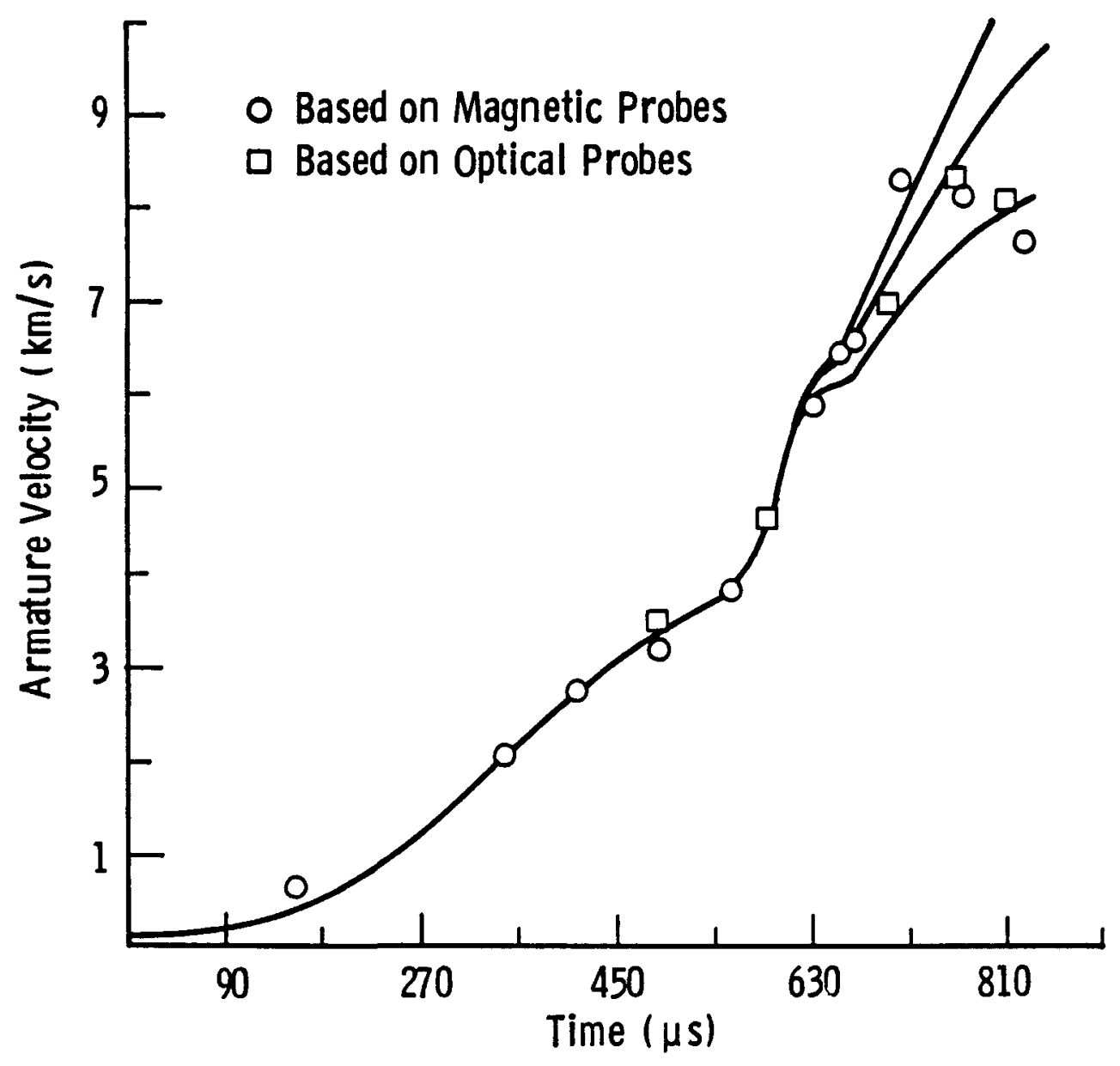

Figure 5.21 - Velocity-time history of plasma armature in Shot OPEN-X1-05. 
The very first B-dot probe exhibits some degree of symmetry of the bipolar signal. This symmetry is a measure of the extent of the localized (confined) nature of the open plasma armature. This symmetry, however, was short-lived. At the 2nd probe position, asymmetry of the signal can be clearly seen. In particular, the second peak in the signal tends to have a lower amplitude, which implies the elongation of the plasma armature breech-wards. The leading edge of the armature is estimated to fly by the probe at approximately $386 \mu \mathrm{s}$. This is about the same order as the characteristic time predicted in Chapter 4 for some instability to grow. Signs of this instability in the plasma tail are quite evident in the signal of probe 3 , and again in probe 5 . At the 6th, 7th, and 8th probes, this instability has transformed into two peaks. A sudden and violent dispersion of the arc seems to have cccurrcd. A secondary arc with a distinct center of current concentration does not appear to have travelled much beyond the 8 th probe, as there is no evidence for a distinct second peak in probes 9, 10,11 , and 12 . It is quite possible that the wake of the plasma has grown so long in these later positions that it has homogenized with the 'secondary arc' to form one long distributed arc.

The violent dispersion of the main arc appears to occur about the same time as the turn-on of the current from the second stage. The current in the bore rises quickly from about $180 \mathrm{kA}$ to about $340 \mathrm{kA}$ in the period between 509 us and $650 \mu \mathrm{s}$. In the same time interval, the armature-projectile appears to experience a big boost in the acceleration (Figures 5.20 and 5.21). The rapid increase of the Lorentz force (factor of 3.6) due to the current rising from $180 \mathrm{kA}$ to $340 \mathrm{kA}$ is the main factor and accounts for about $80 \%$ of the acceleration during this period. We can account for the remaining $20 \%$ of the acceleration if we assume there is a reduction of the parasitic mass of the main arc during this period. To obtain an estimate of this, we proceeded to simulate the experiment computationally. 
For the first $600 \mu \mathrm{s}$, the position-time and velocity-time profiles as measured by the magnetic probes and the optical probes can be simulated with a regular railgun simulation. We assume an effective ablation coefficient of $10 \mathrm{~g} / \mathrm{MJ}$, an arc resistance of $0.5 \mathrm{mn}$, and a skin friction coefficient of 0.002 and an initial arc mass of $150 \mathrm{mg}$. The estimated arc mass as a function of time is shown in Figure 5.22. At the point when the fragmentation of the arc occurs, the armature is estimated to have a mass of $220 \mathrm{mg}$. After the fragmentation, the arc is estimated to have its mass reduced to about $100 \mathrm{mg}$. This smaller arc mass would result in a shorter armature length, and could possibly account for the very much sharper and narrower leading peaks of probes $9,10,11$, and 12 . The broad and slowly diminishing inverse peak of these probes are indicative of an extended distribution of current behind the main body of the first arc.

By assuming that the main arc is sealed behind the projectile, it is inferred that the projectile has the same muzzle velcoity as the arc, which is estimated to be between $8.1 \mathrm{~km} / \mathrm{s}$ and $8.3 \mathrm{~km} / \mathrm{s}$ in this experiment. However, there was no direct measurement of the projectile velocity external to the bore in this experiment. Owing to the richness and the highly complex behavior of the armature, it is highly desirable that experiments such as these be repeated with better in-bore diagnostics and ex-bore measurement of projectile velocity, so that these phenomena can be studied more closely.

\subsection{REMARKS}

The series of experiments conducted on SUVAC has shown that the behavior of the open plasma armatures in railguns is highly complicated, strongly dynamic in nature, and largely uncontrollable in the high velocity domain ( $>$ approximately $6 \mathrm{~km} / \mathrm{s}$ ), though much more experimental work is neccessary to answer many of questions raised here. 
Curve 751916-A

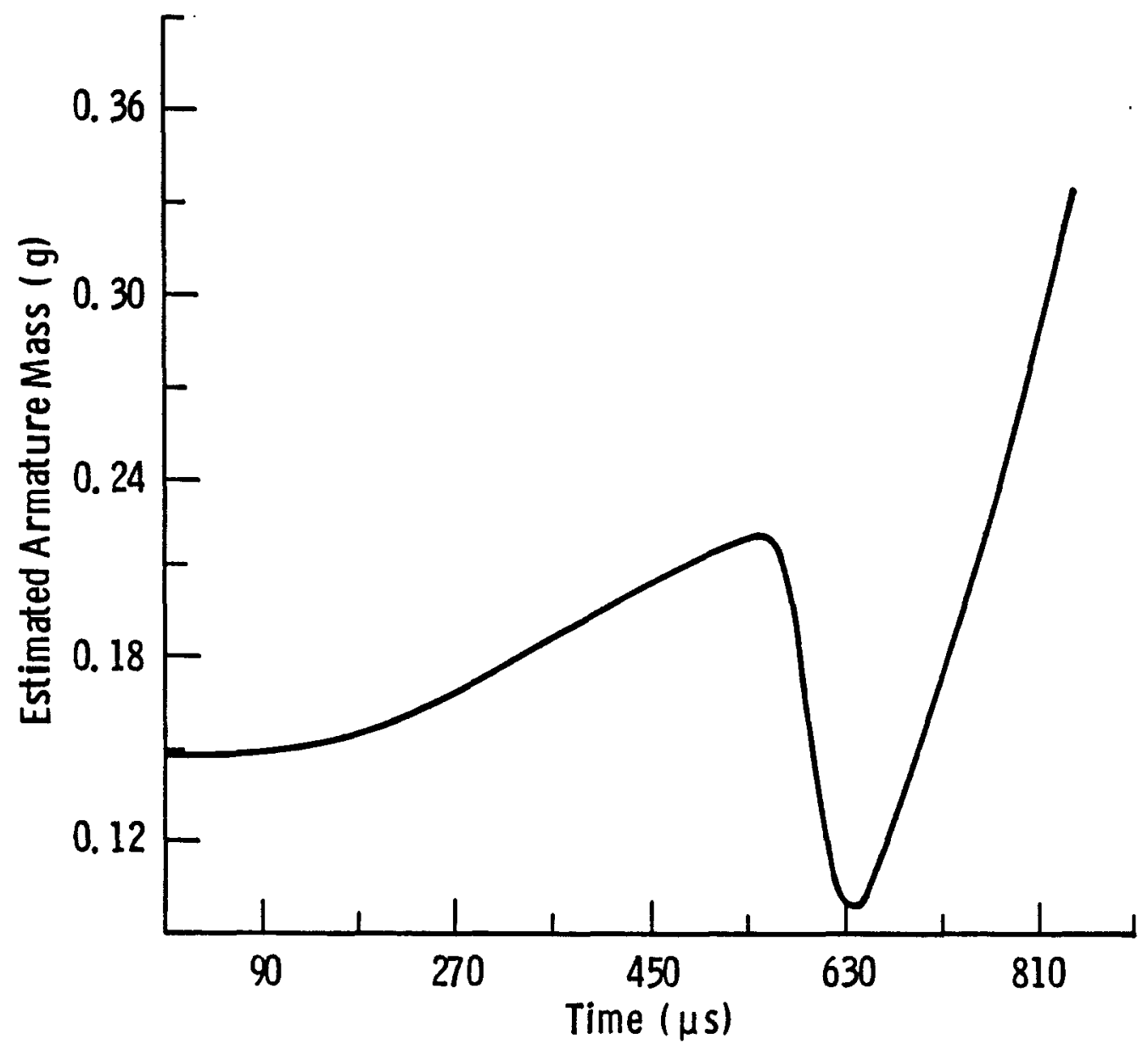

Figure 5.22 - Estimated time history of the armature mass in Shot OPEN-X1-05.

$$
\text { 5-36 }
$$


In order to produce ultrahigh velocity in a reproducible and predictable manner, a plasma armature of a greater degree of controllability is required. In the next chapter, we present one such plasma armature the mechanically controlled plasma armature. 


\section{Mechanically Controlled Plasma Armatures}

\subsection{SUMMARY}

The mechanically controlled plasma armature (the slotted projectile) is developed on this program as an attempt to counter simultaneously the problems of:

(i) Secondary arcs, which includes the problems of

(a) Armature instability and dispersion

(b) Restrike

(ii) Viscous drag

(iii) Ablation of bore insulator

and to help achieve a lower electrical power density in the bore for a given required acceleration by augmenting the Lorentz force with a jet produced thrust. In its role to overcome the problems of secondary arcs and viscous drag, the slotted projectile functions as a 'plasma clipper'.

The effects on the launch dynamics introduced by ablation and viscous drag have been described in previous chapters. The formation of secondary arcs continues to be a main obstacle to reproducible hypervelocity ( $>10 \mathrm{~km} / \mathrm{s}$ ) in railguns. We propose that the formation of a plasma tail and the desorption of gases from the surface of the insulators leading to flash-over are primary causes of the phenomenon, aggravated by secondary causes including the formation of sharp points on the rails, the heated rail surface (increasing thermionic emission and reducing surface insulation), and the resilience of arc electrode spots. 
The new projectile design attempts to mechanically contain the plasma armature, and at the same time, thermally shield the rail spacers insulator from the plasma armature, thereby suppressing the formation of the plasma tail and the desorption of conducting gases from the insulator surfaces. The projectile has a slot (the arc chamber) in it containing the plasma through which the current passes (Figure 6.1). If the rear of the slot is also gas sealed, the pressure build-up in the arc chamber ultimately exceeds the strength of the material. To overcome this difficulty, a nozzle is introduced at the back of the projectile to provide venting of the excess pressure in the plasma chamber; the nozzle is electrically insulated from the rails. During its passage through the nozzle, there is no current passing through the gas. As a result, the gas, which is hot (about $25,000 \mathrm{~K}$ ) on entry into the diverging part of the nozzle, quickly loses its temperature by radiation and supersonic expansion. An overall cooling of the exhaust gas by a factor of about 4 or 5 appears theoretically possible. If so, the temperature of the exhausted gas in the bore of the gun could be brought to below $8000 \mathrm{~K}$, at which temperature the gas would have lost most if not all of its electrical conductivity and secondary arc formation in the exhausted gas can be further suppressed by the presence of electronegative molecules in the gas.

The presence of electronegative molecules in the exhausted gas may be derived from doping the wall of the nozzle or the bore insulator with electronegative substances, or by active injection of

electronegative gas into the bore, or a combination of all of them. For preliminary experimental testing, this could be simply done by spraying the insulator surface and the interior and exterior of the slotted projectile with a teflon coating.

A bonus results from this technique: the exhausted jet provides a thrust to augment the Lorentz force. During the early phase of the 


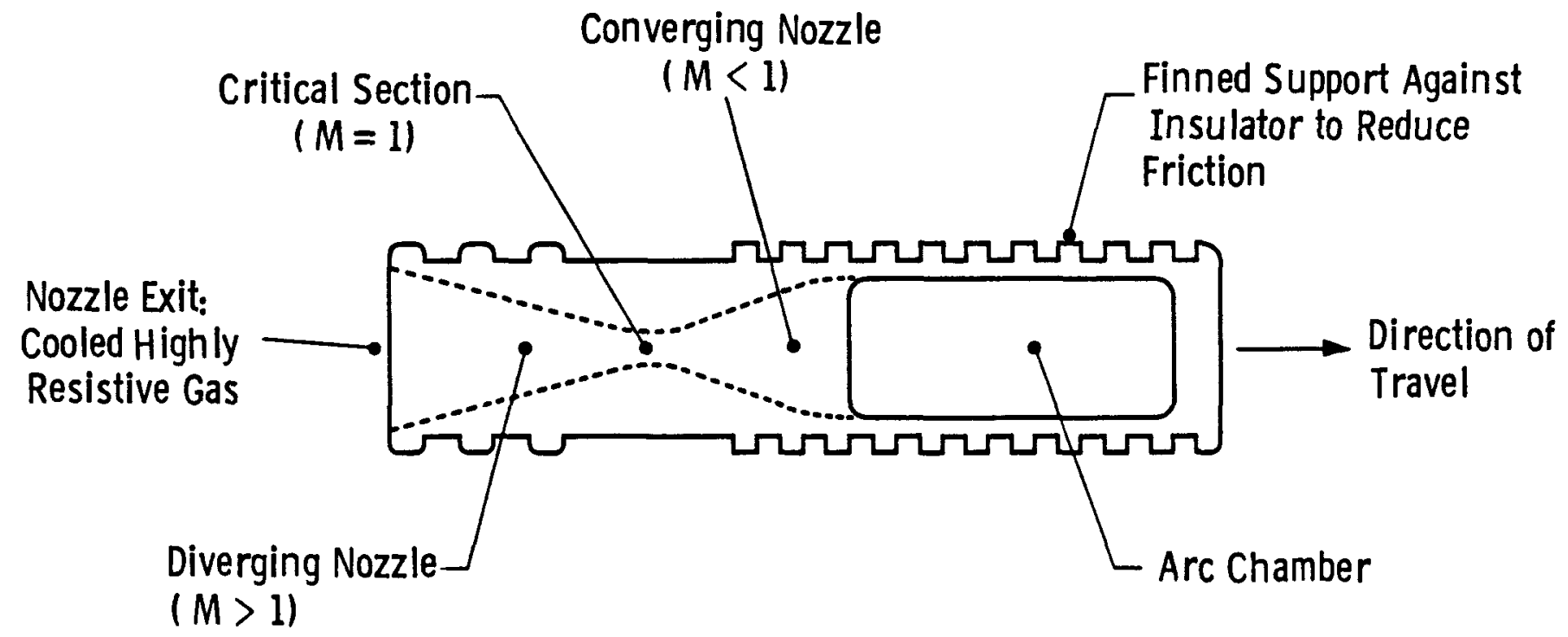

Figure 6.1 - The mechanically controlled plasma armature. 
current build-up when the current is relatively small, the thrust resulting from the jet can be up to several times larger than the Lorentz thrust, thus providing a very desirable initial boost to the projectile having almost the effect of a pre-injector. When the current has built up to its normal operating level, the jet still provides some thrust but typically no more than $20 \%$ of the Lorentz force on the armature.

Using this technique, the ablation of bore insulator can be completely eliminated, and thus solve the critical question on the life of this important and troublesome bore component for the ultimate application where repetitive firing is required. There is no degradation effect on the launch dynamic from the ablation of the plasma chamber wall of the slotted projectile. On the contrary, the ablation of the plasma chamber wall is now an advantage, as it is now used to maintain a conducting arc in the chamber and to provide a required mass flow rate for the jet to produce a given thrust.

In relation to the goal of impact fusion, however, the most important aims of the concept are the control of the viscous drag and of the voltage withstand of the bore immediately behind the moving armature. As we have seen from Section 2, the net viscous drag on the moving plasma armature is proportional to the arc mass. By carefully matching the operating current and the slotted projectile parameters, it is theoretically possible to control the mass of the arc in the chamber as a dynamic balance between the mass ejection rate from the nozzle and the rate of ablation of the plasma chamber. The slotted projectile also provides added design features through which we can gain better control over the conditions of the gaseous products in the bore. This leads to the possibility of achieving through apriori design the required voltage withstand in the bore after the passage of the armature, in contrast to the case of the open armature where the bore conditions after the passage of the armature are largely uncontrollable. In these roles, the slotted projectile acts as a self-synchronised plasma clipper. 
The remainder of this chapter presents the analysis which examines the scientific and engineering feasibility of the concept and its potential application towards achieving ultrahigh velocity (> $20 \mathrm{~km} / \mathrm{s}$ ).

We show that, with the present level of materials technology, a slotted projectile drive system should be capable of achieving velocity in excess of $50 \mathrm{~km} / \mathrm{s}$. Further, if we could make some assumptions regarding material properties based upon projected progress in materials technology over the next decade, it appears very feasible and probable that the slotted-projectile drive systems could reach velocity in excess of $100 \mathrm{~km} / \mathrm{s}$ as required to initiate impact fusion. The system design and operating parameters for achieving this goal are identified for the case of a small bore launcher (Table 6.4).

\subsection{ANALYSIS}

\subsubsection{Overviow}

A steady-state plasma model is used to provide an estimate of the temperature of the plasma inside the chamber. The mass flow rate through the nozzle is set equal to the rate of mass ablation. From a knowledge of the temperature of the plasma in the chamber, the mass flow rate, and a given throat area, the properties (temperature, pressure, density, velocity etc.) through the converging and diverging section of the nozzle are determined, which includes the flow properties at the exit of the nozzle. The flow in the converging nozzle is assumed to be adiabatic (we envisage a short converging section), whereas the flow in the diverging section is analysed for both cases: adiabatic and with heat lost to the wall. The results for the two cases will provide practical bounds on actual flow properties depending on the detailed geometry of the nozzle. For the analysis to be self-consistent, the actual computation is an iterative process giving rise to a converging 
sequence of results from each iteration. The equilibrium arc mass in the arc chamber ( $s \mid o t$ ) is determined from the requirement that the stagnation pressure to sustain a given mass flow rate through the nozzle must be matched by the plasma pressure at the rear of the chamber as calculated from the steady-state plasma armature model. The results of the analysis are used to select sets of technologically feasible designs of the slotted projectile. The launch performance of these technologically feasible designs are then analysed to assess their feasibility or potential of attaining ultrahigh velocity.

\subsubsection{Model for the Plasma in the Arc Chamber}

We assume a constant driving current, and that the arc in the chamber and the flow through the nozzle have reached a steady state. For calculating the state variables of the plasma in the arc chamber, we follow essentially the procedure as described in Thio and Frost [24], but with a few simplifications consistent with the scoping objective of this analysis.

The plasma temperature in the chamber is assumed to be uniform spatially. This is a reasonable assumption in view of the high pressure and high current density of the arc. The plasma in the chamber is essentially a wall-stabilized ablation-dominated arc. The temperature profiles in such arcs are characteristically flat in the interior of the arc except for a very thin boundary layer in contact with the walls. The plasma temperature in the arc chamber is calculated assuming radiative equilibrium between the ohmic dissipation and the radiation from the arc into its surroundings, (see Figure 6.1),

$$
\begin{gathered}
I^{2} R_{A}=\sigma_{s} T^{4}\left(2 \epsilon_{r} h I+2 \epsilon_{i} w l+\epsilon_{a} w h+\epsilon_{b} w h\right) \\
\sigma_{s}=5.67 \times 10^{-8} \mathrm{Wm}^{-2} \mathrm{deg}^{-4} \text { (Stefan's Constant) }
\end{gathered}
$$


where $R_{A}$ is the net resistance of the plasma armature. The resistance of the main body of the arc is estimated in the manner of Thio and Frost. For convenience, we shall also use the value of 1 for all the emissivities occuring in Equation (6.1). For a given current and arc resistance, the arc temperature is evaluated from the above expression. Knowing the temperature, the ionization fractions $a_{i}$ can be evaluated from Saha's equation,

$$
\frac{n_{m+} n_{e}}{n_{m}}=2\left(\frac{2 \pi m_{e} k_{B} T}{h^{2}}\right]^{3 / 2} \frac{U_{m+1}}{U_{m}} \exp \left[-\frac{e\left(V_{m}-\Delta V_{m+1}\right)}{k_{B} T}\right]
$$

where $U_{m}$ and $\Delta V_{m}$ are the partition function and the lowering of ionization potential due to non-ideal effects respectively of the ion in the $\mathrm{m}$-th state of ionization. For the present representative analysis, we shall use only $\mathrm{N}$ ions or $\mathrm{N}-\mathrm{like}$ ions for illustration.

For these ions and the temperature range we shall encounter, second ionization occurs only in negligible proportion. For the first ionization, the above Saha equation simplifies to

$$
\frac{a^{2}}{1-a}=2\left(\frac{2 \pi m_{e} k_{B} T}{h^{2}}\right)^{3 / 2}\left(\frac{U_{1}}{U_{0}}\right) \frac{1}{n_{T}} \exp \left[-\frac{e\left(V_{1}-\Delta V_{1}\right.}{k_{B} T}\right]
$$

where $n_{T}$ is the total (neutrals and ions) particle number density and may be approximated by its mean value for the chamber,

$$
n_{T}=\frac{m_{A}}{m_{P}} \frac{1}{w h l}
$$

and $u_{0} / u_{1} \sim 1 / 2$. The current is assumed to distribute uniformly in the arc chamber. This is a reasonable assumption for such a high-pressure, relatively high-temperature, confined arc. The magnetic field, pressure and density, however, are allowed to vary in the chamber in a 1-D fashion axially along the bore. (See Figure 6.2) 
Dwg. $9385 A 76$

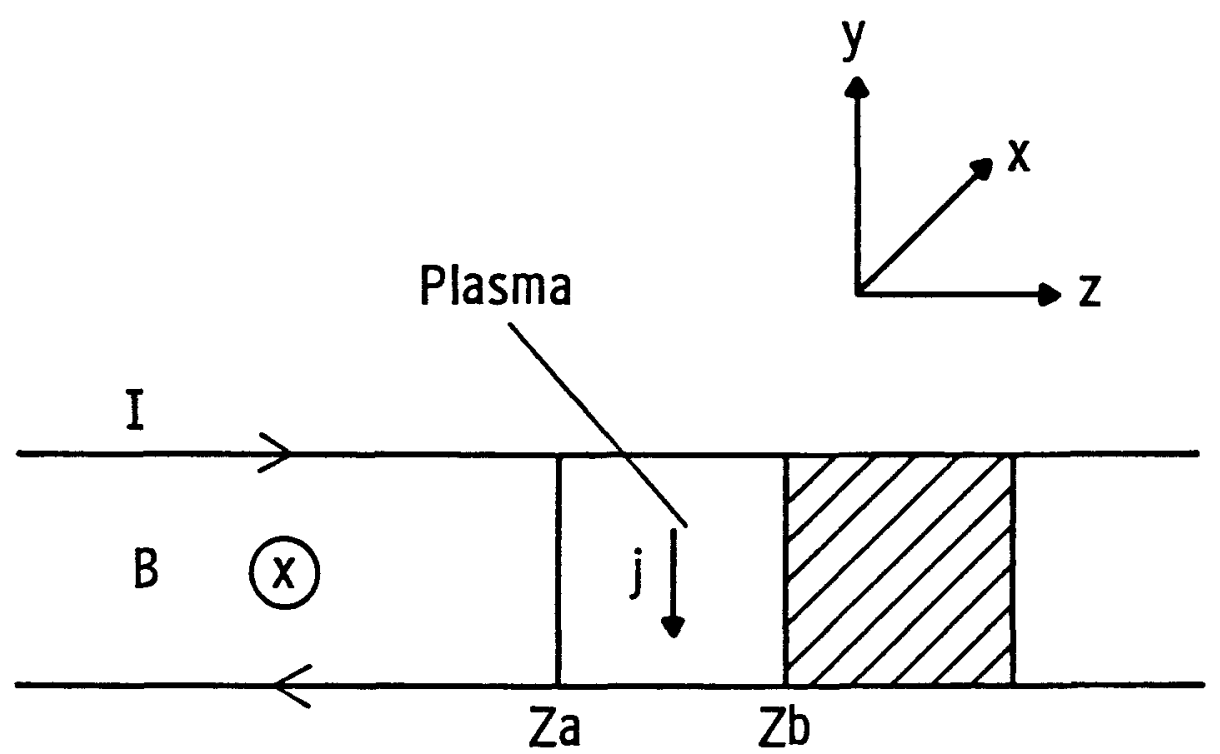

Figure 6.2 - Geometry of the model of the plasma in the arc chamber. The geometries of the chamber and nozzle are not shown. 
We can easily integrate Maxwell's equation and the steady-state magnetogasdynamic equation given as follows,

$$
\begin{gathered}
\frac{\partial H}{\partial z}=-j \\
\rho a+\frac{d p}{d x}=j B
\end{gathered}
$$

to obtain the pressure distribution in the chamber. The result is

where

$$
p(z)=\frac{\mu i^{2}}{\left[k_{1} a\right)^{2}}\left\{b_{1}\left(1-e^{-k_{1} a \zeta}\right)-k_{1} a \zeta\right\}+p_{a} e^{-k_{1} a \zeta}
$$

$$
\begin{aligned}
& b_{1}=\frac{B\left[z_{a}\right] k_{1} a}{\mu j}+1 \\
& k_{1}=\frac{m_{p}}{(1+a) k_{B} \top}
\end{aligned}
$$

and $S=z-z_{a}, z_{a}$ is the $z$-coordinate of the rear of the arc chamber, and $\mathrm{P}_{\mathrm{a}}$ is the pressure there. i.e. entrance to the converging nozzle (see Figure 6.1). The acceleration of the total payload is $a, k_{B}$ is the Boltzmann constant, and $m_{p}$ is the mass of the plasma atomic species, $p$ is the local density of the plasma, and $T$ is the arc temperature. The current density $j=I / / h$ where $l$ is the length of arc chamber, and $h$ is the height of the slot through which current passes between the rails. Implicitly, we have assumed that the arc current (but not the gaseous material comprising the arc) has been confined to the arc chamber, and that no current flows in the nozzle due to its insulating walls. The validity of this assumption can only be verified by experimentation. 
The ideal plasma equation of state has been used,

$$
p=(1+\alpha) \frac{\rho k_{B} T}{m_{p}} \quad k_{B}=1.38 \times 10^{-23} \mathrm{~J} / \text { deg } K
$$

Using the equation of state and the Expression (6.3) for pressure, the mass density distribution in the arc chamber can be calculated, from which an expression for the arc mass is obtained,

where

$$
m_{A}=\left(\frac{\mu w}{2 h}\right)\left(\frac{b_{2}}{b_{3}}\right) T^{-1} I_{I^{2}}{ }^{2}+\frac{P_{a} A_{b}}{a}\left[1-e^{-\rho)}\right)
$$

$$
\begin{gathered}
b_{2}=\frac{1}{\beta^{2}}\left\{2 b_{1}\left[1+\frac{1}{\beta}\left(e^{-\beta}-1\right)\right]-\beta\right\} \\
b_{3}=(1+a) k_{B} / m_{p} ; A_{b}=w h \\
\beta=k_{1} a l=\frac{m_{p} a l}{(1+a) k_{B} \top}
\end{gathered}
$$

we note that, in the limit as the acceleration a $\rightarrow 0$, the expression $b_{2}$ tends to a corresponding limit given by,

$$
b_{2}+\frac{B_{a}}{\mu j l}-\frac{1}{3}
$$

In the computation, the above expressions are used to determine the pressure $B_{a}$ at the nozzle entrance corresponding to a given arc mass. The magnetic field at the "rear boundary" (nozzle entrance) of the arc chamber is given by Thio and Frost as,

$$
B_{a}=\left(\frac{h L^{\prime} \text { eff }}{2 \mu w}-\frac{1}{2}\right) \frac{\mu I}{h}
$$

where $L^{\prime}$ eff is the effective inductance gradient of the accelerator and $w$ is the rail separation. 


\subsubsection{Adiabatic Flow Through the Nozzle}

Consider the control volumes as shown in Figure 6.3. Section 0 represents the stagnation point. Because of the low ambient pressure at the exit in the bore, we expect the flow to become supersonic at exit, so that there is a sonic point in the nozzle where the flow velocity coincides with the local sound speed. The sonic point is a critical point and is indicated in Figure 6.3 as section 2 in the flow.

The temperature $T$ of the plasma in the arc chamber is taken as the stagnation temperature $T_{0}$ for the flow throught the nozzle.

For a general section (labelled as $k$ in Figure 6.3), if the flow in the control volume between it and section 0 is adiabatic, then conservation of energy in the control volume gives,

$$
h_{0}=h_{k}+\frac{u_{k}^{2}}{2}
$$

where $h_{0}, h_{k}$ are enthalpy of the gas at section 0 and section $k$ respectively, $u_{k}$ is the flow velocity at section $k$. For a monoatomic singly ionized ideal plasma, the enthalpy of the plasma at a temperature $T$ may be taken as,

$$
h=\frac{5}{2}(1+a) R T+a e_{I}
$$

where $e_{I}$ is the ionization energy per unit mass of the atomic species making up the plasma. Using the expression for the local sound speed $c_{s}=\left(R^{\prime} T\right)^{1 / 2}$ and the above expression for the enthalpy, we may solve the energy equation to give the temperature $T_{k}$ at section $k$ in terms of the local Mach number $M_{k}$ as,

$$
T_{k}=\frac{5\left(1+a_{0}\right) R T_{0}+2\left(a_{0}-a_{k}\right) e_{I}}{R\left(1+a_{k}\right)\left(5+\gamma_{k} M_{k}^{2}\right)}
$$






Figure 6.3 - Showing the control volumes used in analyzing the behavior of the mechanically controlled plasma armature. Section 0 represents the stagnation point of the flow. Section 2 represents the critical section at which the local Mach Number is 1 . Section 3 represents the exit of the nozzle. Section $K$ represents a general section in the nozzle. 
where $\gamma_{k}$ is the ratio of specific heats, $c_{p} / c_{v}$. The gas velocity at the section is simply given by,

$$
u_{k}=M_{k} \sqrt{\gamma_{k}^{R}\left(1+a_{k}\right) T_{k}}
$$

In steady state, the mass flow rate through the nozzle must equal to the rate of ablation by the arc in the arc chamber. Thus,

$$
G=a_{b} I^{2} R_{A}
$$

where $a_{b}$ is the effective ablation coefficient.

Select an area for the critical section, i.e., the sonic point. Let the area be denoted by $A_{2}$. For flow continuity through this section we require the density at the section $\rho_{2}$ to be given by,

$$
\rho_{2}=\frac{G}{U_{2} A_{2}}
$$

For the determination of the flow properties at a general section in the nozzle, the adiabatic (isentropic) condition of the flow needs to be incorporated. The adiabatic requirement in general imposes upon the flow some relationships between the pressure and the density in addition to the equation of state. Caution must be exercised here, since we are dealing with an ionized flow with rapidly changing degree of ionization. In an ionized flow the specific heats $c_{v}$ and $c_{p}$ are functions of the degree of ionization. Fortunately, for the flow under investigation, their ratio is only a slowly varying function of the flow. Under these conditions, the adiabatic relationships between pressure and density, temperature and density can be approximately taken to be, 


$$
\begin{gathered}
\frac{P}{P_{2}}=\frac{\rho \gamma}{\rho_{2} \gamma_{2}} \\
\frac{\rho_{1} \gamma_{1}^{-1}}{\rho_{2} \gamma_{2}^{-1}}=\frac{\left(1+\alpha_{1}\right) T_{1}}{\left(1+\alpha_{2}\right) T_{2}}
\end{gathered}
$$

Using these relationships, we can express the flow at a general section in terms of the flow properties at the critical (sonic) section as follows. Given the Mach number at the section, Expression (6.9) is used to calculate its temperature, assuming a value for the local degree of ionization $a_{k}$. Expression (6.13b) is then used to calculate the density at the section. With these tentative values of the temperature and the density, Saha equation can now be used to determine the local degree of ionization at the section. The new value for $a_{k}$ is then used to update the temperature and the density. This sequence of computation is repeated until convergence is obtained. Knowing the density, the continuity condition now requires the area of the section to be given by,

$$
A=\frac{G}{\rho u}
$$

where the flow velocity has been determined from the specified local Mach number and the temperature at the section,

$$
\mu=M \sqrt{\gamma(1+a) R T}
$$

This completes the ensemble of relationships for determining any flow properties throughout the nozzle if adiablatic condition is assumed. In the next section we show how the results can be modified to calculate the exit flow properties if some heat loss to the wall can be assumed to occur. 


\subsubsection{Flow in The Diverging Section with Heat Loss}

After passing through the throat, the gas in general is still very hot, and has a relatively high degree of ionization. The energy associated with ionization could account for a substantial fraction of the enthalpy of the gas. We assume for the moment that the geometry of the diverging nozzle can be designed so that heat loss by radiation and convection to the wall is sufficient to remove the energy released by the recombination of the ions and electrons. Mathematically, this is expressed by the following relationship,

$$
d q=\left[\frac{3}{2} R T+e_{I}\right] d a
$$

We shall further assume, however, that the diverging part of the nozzle is sufficiently long by design that, on exit from the nozzle, the temperature of the gas on exit is low enough that the degree of ionization $\alpha_{3}$ at exit is very much smaller than the degree of ionization at the critical point.

With reference to Figure 6.3, energy balance over the control volume between section 2 and 3 gives,

$$
\frac{1}{2} R T_{3}\left(5+\gamma_{3} M_{3}^{2}\right)=\frac{1}{2} R\left(1+\alpha_{2}\right)\left(5+\gamma_{2}\right) T_{2}-\frac{3}{2} R \alpha_{2} T
$$

where $T$ is a mean temperature over the control volume defined as follows,

$$
T=\frac{1}{\left(a_{3}-a_{2}\right)} \int_{a_{2}}^{a_{3}} T d a
$$


It is sufficient for the present purpose to approximate the mean temperature $T$ as $\left(T_{3}+T_{2}\right) / 2$. With this approximation, the above Expression (6.17) can be manipulated to give an expression for the temperature $T_{3}$ of the gas at exit in terms of its temperature $T_{2}$ at the critical section and the local Mach number,

$$
\frac{T_{3}}{T_{2}}=\frac{\left(1+a_{2}\right)\left(5+\gamma_{2}\right)-\frac{3}{2} \alpha_{2}}{\left(5+\gamma_{3}\right) M_{3}^{2}+\frac{3}{2} a_{2}}
$$

Locally, the energy equation is,

$$
\mathrm{dq}=\left(\frac{3}{2} \mathrm{RT}+\mathrm{e}_{\mathrm{I}}\right) \mathrm{d} \alpha=\frac{3}{2} \mathrm{R}(1+\alpha) \mathrm{dT}-(1+\alpha) \frac{\mathrm{RT}}{\rho} \mathrm{d} \rho
$$

Integrating the resulting differential equation, we obtain a relationship between the local gas density and temperature as,

$$
\frac{\rho}{\rho_{2}}=\left[\frac{T}{T_{2}}\right]^{3 / 2}
$$

This is similar in form to a corresponding relationship for an isentropic flow without ionization.

Expressing the condition for the continuity of the flow across the sections 2 and 3 in terms of the local Mach number and using the above relationship between the density and temperature, the following relationship between the cross sectional area at the exit and the critical section can be shown to hold,

$$
\frac{A_{3}}{A_{2}}=\frac{\left(1+\alpha_{2}\right)^{1 / 2}}{M_{3}\left(T_{3} / T_{2}\right)^{2}\left(\gamma_{3} / \gamma_{2}\right)^{1 / 2}}
$$

The two relationships (6.19) and (6.22) can be used to select a ratio of the exit area to the area of the sonic section to produce a given temperature ratio. Or, conversely, if the area ratios are fixed a priori, we can determine the temperature ratio and the Mach number 
from these two relationships. The density and pressure ratios can also determined using Equation (6.21) and the equation of state.

We see from here an important feature of the slotted projectile concept. The concept provides additional engineering design constructs by which we can gain control over the condition of the gaseous products left behind by the moving plasma armature. The total lack of control of the wake of the open plasma armature is a source of many problems of using the open plasma armature in railguns. Worse, the lack of precise knowledge of the post-firing gaseous conditions in the bore after the passage of an open plasma armature makes the designing of reliable repetitive firing systems an almost impossible engineering task.

\subsection{FEASIBILITY: A CONCEPTUAL, POINT DESIGN ANALYSIS}

We shall now work out the above analysis for the case of a nitrogen plasma as an example. Other plasmas such as $H, 0, L i$ or $C$ can also be considered. The systematic search for the optimum species or combination of them and their practical realization is beyond the scope of the present study. We merely wish to investigate here the feasibility of the concept and have selected to use the $N$ plasma as an example because its ionic properties are representive of many plasmas which may be implemented in practice. The first ionization energy per unit mass for nitrogen atoms is $100.2 \mathrm{~J} / \mathrm{mg}$. Let us pick an arc chamber which is $1 \mathrm{~cm} \mathrm{high,} 1 \mathrm{~cm}$ wide (separation between rails) and $3 \mathrm{~cm}$ long. Also, let us start with an arc mass of $50 \mathrm{mg}$ in the arc chamber. Let the inductance gradient L' of the gun be $1.2 \mu \mathrm{H} / \mathrm{m}$ and let the current be $200 \mathrm{kA}$. We assume that the resultant slotted projectile would have a mass of about $3.5 \mathrm{~g}$. The nominal acceleration of the projectile can be calculated to be $8.23 \times 10^{6} \mathrm{~m} / \mathrm{s}^{2}\left[\left(1 / 2 \mathrm{~L}^{\prime} \mathrm{I}^{2} / \mathrm{m}\right)(1+\beta)\right.$ where $\beta$ is the boost factor due to the jet].

The Thio-Frost formulation is used to estimate the arc resistance. Specializing Thio-Frost [24] formulation to the case of a singly ionized oxygen plasma, the electrical resistivity of the plasma is given as, 


$$
\eta_{p}=\eta_{e i}+\eta_{e n}
$$

where,

$$
\begin{gathered}
\eta_{e i}=3.5 \times 10^{2} S_{T} \exp (-2 \gamma / 3 Z) T^{-3 / 2} \\
\gamma / Z=e^{2} n_{c}^{1 / 3} / 4 \pi \epsilon_{o} k_{b} T ; n_{c}=n_{e}+n_{i} \\
\eta_{e n}=\left(\frac{m_{e}}{2}\right)\left(\frac{1-a}{a}\right) S_{o} T^{1 / 2}
\end{gathered}
$$

The values for $s_{0}$ are $0.57 \times 10^{-15}, 4.4 \times 10^{-15}, 1.3 \times 10^{-15}, 6.2 \times$ $10^{-15}$ for a pure $0, H, N$ and Li plasma respectively.

The arc resistance is then calculated from the familiar formula,

$$
R_{A}=\frac{\eta_{p} w}{h L}
$$

Equations (6.23) and (6.24) togerther with Equations (6.1) and (6.2b) are solved simultaneously to obtain the resistance, the temperature, and the degree of ionization of the plasma in the arc chamber. The resistance of the plasma-rail boundary layer (dominated by arc roots formation) is estimated to be of the order of $.05 \mathrm{mn}$ to $.1 \mathrm{~m} \Omega$ (but see also Marshall[33]). This is added to resistance of the arc column to obtain the total arc resistance.

For the case considered, the temperature of the plasma in the arc chamber is estimated to be approximately $30,500 \mathrm{~K}$, the total arc resistance $1.7 \mathrm{mn}$, and the degree of ionization to be 0.23 . The magnetic field at the rear of the arc chamber is given by Expression (6.6) to be $24.6 \mathrm{~T}$. We have now all the data required for evaluating the pressure at the rear of the arc chamber from Equation (6.5a), which is found to be $203 \mathrm{MPa}$. The density there is $9.1 \mathrm{~kg} / \mathrm{m}^{3}$. 
With the calculated value of $30,500 \mathrm{~K}$ as the stagnation temperature and assuming an effective ablation coefficient of $7.5 \mathrm{~g} / \mathrm{MJ}$, the flow properties in the sonic section of the nozzle can be computed. In particular, the results give the temperature there to be approximately $27,000 \mathrm{~K}$ if the sonic section is chosen to have a radius of $1.6 \mathrm{~mm}$. The pressure at various sections of the converging nozzle can be calculated using the analysis described above. Extrapolating the calculated pressure backward towards the entrance of the nozzle, it is found that the "stagnation" pressure required to sustain the flow is approximately $310 \mathrm{MPa}$. This is higher than the pressure calculated for the rear of the arc chamber (203 MPa) assuming an arc mass of $50 \mathrm{mg}$, showing that the assumed arc mass in the chamber is not sufficient to maintain the flow rate through the nozzle. To obtain a pressure at the rear of the chamber consistent with the stagnation pressure for the nozzle flow, the arc mass in the chamber has to be increased to $63 \mathrm{mg}$. This is the equilibrium arc mass for the system. With this arc mass, the pressure at the front of the arc chamber is found to be $550 \mathrm{MPa}$. This is a relatively high pressure in terms of the state of the art of sabot technology, though it is quite possible that progress in the sabot technology in the future could contain these high pressures.

It is seen here how the arc mass in the chamber is determined from the design of the slotted projectile, and the operating parameters. We shall see later the importance of being able to control the arc mass in order to attain the velocity of interest to impact fusion.

In terms of current sabot and launcher containment technology, maintaining the gas pressures in the chamber to be below $350 \mathrm{MPa}$ (50,000 psi) is desirable. This can be realized for the following combination of design and operating parameters: a current of $170 \mathrm{kA}$, an $L^{\prime}$ of $1.2 \mu \mathrm{H} / \mathrm{m}$, a chamber width and height of $1 \mathrm{~cm}$, a chamber length of $3 \mathrm{~cm}$, and an effective ablation coefficient of about $5 \mathrm{~g} / \mathrm{MJ}$. The resultant equilibrium arc mass is found to be $41 \mathrm{mg}$. The arc 
temperature in the chamber is found to be about $28,800 \mathrm{~K}$, the resistance $1.9 \mathrm{~m}$, and the degree of ionization 0.21 . The pressure at the rear of the chamber is found to be about $160 \mathrm{MPa}$, with a density of $7.6 \mathrm{~kg} / \mathrm{m}$ and a specific enthalpy of $73.26 \mathrm{~J} / \mathrm{mg}$ there. The pressure at the front of the chamber is $340 \mathrm{MPa}$, desirably below the current technology threshold.

The flow properties in the converging nozzle in this case is shown in Table 6.1. Table 6.2 shows the flow properties in the diverging section of the nozzle if the flow is assumed adiabatic, while Table 6.3 shows the flow properties in the same diverging section if heat loss to the wall in the fashion described in Section 6.2.4 is assumed.

In the converging part of the nozzle, the flow is more or less "frozen" with respect to the degree of ionization and the temperature. It is seen that the temperature stays nearly constant at about $26000 \mathrm{~K}$ while the degree of ionization is nearly frozen at 0.2 . The stagnation pressure is nearly $165 \mathrm{MPa}$ which is consistent with the pressure at the rear of the arc chamber produced by the magnetic and thermal pressure of the arc. The pressure in the throat (the sonic section) is about $120 \mathrm{MPa}$, a modest pressure which is relatively easy to contain. The sound and flow velocity reaches a value of nearly $5 \mathrm{~km} / \mathrm{s}$ here.

If there is no heat transfer to the wall of the nozzle, the flow properties are shown in Table 6.2. For the gun used in the example the bore would have a square crossection which is approximately $1.2 \mathrm{~cm}$ high. The effective radius of the nozzle at exit is about $5.5 \mathrm{~mm}$. We see from Table 6.2 that with this exit radius a flow Mach number of 5 could possibly be achieved at the exit. The corresponding temperature of the gas at exit could be as low as $5300 \mathrm{~K}$. The gas has virtually lost all of $i$ ts ionization. The electrical resistivity of the gas is estimated 
Table 6.1 - Flow Properties in the Converging Nozzle

\begin{tabular}{|c|c|c|c|c|c|c|}
\hline $\begin{array}{l}\text { Section radius, } r(\mathrm{~mm}) \\
\text { Temperature, } T\left(10^{3} \mathrm{~K}\right) \\
\text { Ionization fraction, } \\
\text { Density, }\left(\mathrm{kg} / \mathrm{m}^{3}\right) \\
\text { Pressure, } \mathrm{P}(\mathrm{MPa}) \\
\text { Sound speed, } c(\mathrm{~km} / \mathrm{s}) \\
\text { Mach number, } M \\
\text { Flow velocity, u }(\mathrm{km} / \mathrm{s}) \\
\text { Particle density }\left(10^{26}\right) \\
\text { Ratio of sp. heats, } \\
\text { Specific heat, c }(\mathrm{J} / \mathrm{g}) \\
\text { Specific heat, c }(\mathrm{J} / \mathrm{g}) \\
\text { Resistivity, }\left(10^{-5} \mathrm{~nm}\right)\end{array}$ & $\begin{array}{l}4.44 \\
27.5 \\
0.23 \\
8.19 \\
165 \\
5.41 \\
0.1 \\
0.54 \\
3.53 \\
1.46 \\
4.54 \\
6.62 \\
5.65\end{array}$ & $\begin{array}{l}3.15 \\
27.4 \\
0.23 \\
8.15 \\
163 \\
5.40 \\
0.2 \\
1.08 \\
3.51 \\
1.46 \\
4.53 \\
6.60 \\
5.69\end{array}$ & $\begin{array}{c}2.26 \\
27.1 \\
0.22 \\
7.98 \\
156 \\
5.34 \\
0.4 \\
213 \\
3.43 \\
1.45 \\
4.50 \\
6.53 \\
5.86\end{array}$ & $\begin{array}{l}1.90 \\
26.5 \\
0.21 \\
7.70 \\
146 \\
5.24 \\
0.6 \\
3.15 \\
3.31 \\
1.45 \\
4.44 \\
6.42 \\
6.13\end{array}$ & $\begin{array}{l}1.71 \\
25.8 \\
0.19 \\
7.32 \\
134 \\
5.12 \\
0.8 \\
4.10 \\
3.15 \\
1.44 \\
4.36 \\
6.26 \\
6.53\end{array}$ & $\begin{array}{l}1.60 \\
24.9 \\
0.17 \\
6.85 \\
119 \\
4.98 \\
1.0 \\
4.98 \\
2.95 \\
1.43 \\
4.25 \\
6.06 \\
7.06\end{array}$ \\
\hline
\end{tabular}

Table 6.2 - Flow Properties in the Diverging Nozzle Assuming Adiabatic Condition

\begin{tabular}{|c|c|c|c|c|c|c|c|}
\hline 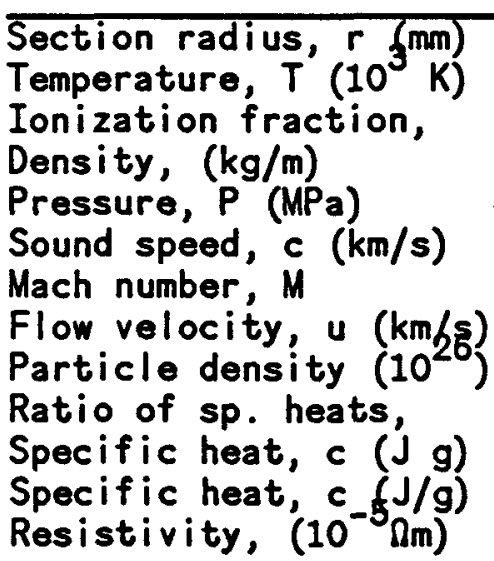 & $\begin{array}{c}1.69 \\
19.4 \\
.078 \\
3.71 \\
46.2 \\
4.13 \\
2.0 \\
8.26 \\
1.60 \\
1.37 \\
3.31 \\
4.54 \\
12.9\end{array}$ & $\begin{array}{l}2.07 \\
16.5 \\
.043 \\
2.19 \\
22.3 \\
3.73 \\
2.5 \\
9.32 \\
0.94 \\
1.37 \\
2.64 \\
3.61 \\
20.5\end{array}$ & $\begin{array}{l}2.73 \\
13.5 \\
.016 \\
1.15 \\
9.36 \\
3.38 \\
3.0 \\
10.1 \\
0.49 \\
1.41 \\
1.85 \\
2.60 \\
43.0\end{array}$ & $\begin{array}{l}3.51 \\
10.2 \\
.0024 \\
0.66 \\
4.0 \\
3.07 \\
3.5 \\
10.8 \\
0.28 \\
1.55 \\
1.13 \\
1.75 \\
208\end{array}$ & $\begin{array}{r}4.11 \\
7.87 \\
2 \times 10^{-4} \\
0.47 \\
2.18 \\
2.77 \\
4.0 \\
11.1 \\
0.20 \\
1.65 \\
0.92 \\
1.52 \\
2060\end{array}$ & $\begin{array}{c}4.73 \\
6.38 \\
1.6 \times 10^{-} \\
0.35 \\
1.31 \\
2.51 \\
4.5 \\
11.3 \\
0.15 \\
1.66 \\
0.89 \\
1.49 \\
2 \times 10^{4}\end{array}$ & $\begin{array}{c}0.82 \\
2.29 \\
5.0 \\
11.4 \\
0.11 \\
1.67 \\
0.89 \\
1.48 \\
3 \times 10^{5}\end{array}$ \\
\hline
\end{tabular}

Table 6.3 - Flow Properties at Exit with Heat Loss in the Diverging Nozzle

\begin{tabular}{lccc}
\hline Section radius, r (mm) & 3.52 & 4.05 & 4.62 \\
Temperature, T' (10 $\mathrm{K}$ ) & 5.67 & 4.64 & 3.86 \\
Ionization fraction, & $1.9 \times 10^{-6}$ & $7.2 \times 10^{-8}$ & $1.8 \times 10^{-9}$ \\
Density, (kg/m) & 0.74 & 0.52 & 0.42 \\
Pressure, P (MPa) & 2.51 & 1.52 & 0.96 \\
Sound speed, c (km/s) & 2.37 & 2.15 & 1.96 \\
Mach number, M & 4.0 & 4.5 & 5.0 \\
Flow velocity, u (km/s) & 9.49 & 9.66 & 9.78 \\
Particle density (10 & 0.32 & 0.24 & 0.18 \\
Ratio of sp. heats, & 1.67 & 1.67 & 1.67 \\
Resistivity, (10 & 1.79 & 43.4 & 1585 \\
Paschen Voltage (kV) & 47 & 34 & 25 \\
Jet Thrust (kN) & 2.6 & 2.7 & 2.7 \\
Jet to Lorentz ratio & 0.15 & 0.15 & 0.16 \\
\hline
\end{tabular}


at 5 orders of magnitude higher than the plasma in the arc chamber. If the exhausted gas has accumulated some electronegative molecules in its passage through the nozzle, we could reasonably expect an extremely low probability of the formation of secondary arcs from the exhausted gas. In fact, because of the relatively high pressure of the exhausted gas (about 10 atmosphere), the corresponding Paschen break-down voltage is relatively high, of the order of tens of kilovolts. Of course, the applicability of the common Paschen curve to the present case of an heated gas may be questionable. Nevertheless, the high pressure of the exhausted gas should be a deterrent to secondary break-down of the bore behind the armature.

If the heat loss to the wall of the nozzle can be made to occur at sufficiently high rate as to satisfy Equation 6.16, the properties of the gas at the exit of the nozzle are shown in Table 6.3. The main difference introduced by the heat exchange is that the gas at exit is considerably cooler. At the exit radius of $4.7 \mathrm{~mm}$ the gas expansion resulted in a exit Mach number of 5 and a temperature as low as $3900 \mathrm{~K}$. At this temperature, ionization has virtually disappeared (has a value of $2 \times 10^{-9}$ ). The corresponding resistivity of the gas is about 8 orders of magnitude higher than that of the plasma in the arc chamber. The Paschen break-down voltage estimated at the pressure of the exhausted gas is about $25 \mathrm{kV}$, sufficiently high to withstand the back emf from the moving armature up to at least $50 \mathrm{~km} / \mathrm{s}$ for the present design, in which the driving current is $170 \mathrm{kA}$ and the inductance gradient L' is $1.2 \mu \mathrm{H} / \mathrm{m}$.

\subsection{LAUNCH DYNAMICS OF MECHANICALLY CONTROLLED PLASMA ARMATURE}

\section{Comparison with Open Plasma Armature}

In this section, we present a comparative study of the launch dynamics of the mechanically controlled plasma armature (MCPA) and that 
of the open plasma armature in terms of their potential to reach velocity required for impact fusion. To keep the mathematical details to the minimum, we shall present the study for the case of a constant current drive.

In the case of the slotted projectile, with a constant current drive and after an initial transient when the flow through the nozzle has reached a steady state, its equation of motion takes a particularly simple form, since the mass of the accelerating payload is almost constant, if we neglect the mass loss due to the jet, again for mathematical convenience. Thus,

$$
\frac{d}{d t} m_{T} u=(1+\beta) F_{1}-2 \int_{0}^{l} \frac{1}{2} c_{f} \rho u^{2} h d z
$$

where $m_{T}$ and $u$ are the instantaneous mass and velocity of the ensemble, $F$ is the Lorentz force $1 / 2 L^{\prime} I$, and $\beta$ is the boost factor giving the thrust from the jet. Taking account of the mass loss from the ensemble would further enhance the launch dynamics, so that the results presented here may be considered as an worst-case analysis. Expressing the velocity, displacement and time in terms of their dimensionless equivalents defined as follows,

$$
\begin{aligned}
& u=\sqrt{\epsilon} \quad u_{F} \quad u_{F}=\int \frac{2(1+\beta) I_{H} F_{L}}{c_{f} m_{A}}, I_{H}=\frac{w}{2} \\
& S=x / I_{0} \quad I_{0}=\frac{m_{T} u_{F}^{2}}{2(1+\beta) F_{I}} \\
& \tau=t / t^{1} \quad t^{1}=\frac{m_{T} u_{F}}{(1+\beta) F_{1}}
\end{aligned}
$$


the above equation may be manipulated to give the following fundamental pair of equations governing the motion of the system in space and time,

$$
\begin{aligned}
& \frac{d \epsilon}{d \zeta}=1-\epsilon \\
& \frac{d Q}{d \tau}=1-\theta^{2}
\end{aligned}
$$

Their solutions are given respectively as,

$$
\begin{gathered}
\epsilon=1-\left(1-\epsilon_{0}\right) \epsilon^{-\zeta} \\
Q=\frac{1-b e^{-2 \tau}}{1+b e^{-2 \tau}} \quad b=\frac{1-\theta_{0}}{1+\theta_{0}}
\end{gathered}
$$

where $\epsilon_{0}$ is the value of $\epsilon$ when $\zeta=0$, and $\theta_{0}$ is the value of $\theta$ when $\tau=0$.

In Figures 6.4 and 6.5, we show the performance of the slotted projectile system as calculated from the above expressions. In Figure 6.4, the performance is shown by the curve indicated as MCPA-1 for the armature-projectile-launcher system based upon the design described in the previous section. That is, a launcher system is selected with an $L^{\prime}$ of $1.2 \mu \mathrm{H} / \mathrm{m}$ and a driving current of $170 \mathrm{kA}, a$ slotted projectile with a mass of $3 \mathrm{~g}$ carrying an arc mass of $41 \mathrm{mg}$, a bore of approximately $1.2 \mathrm{~cm}$ in height and $1 \mathrm{~cm}$ wide (rail separation), an effective ablation coefficient of $5 \mathrm{~g} / \mathrm{MJ}$ giving a boost factor of 0.16 . The skin friction coefficient is conservatively assumed to be 0.001 . With this system, the maximum velocity, which is limited only by the viscous drag on the arc, is around $70 \mathrm{~km} / \mathrm{s}$, but requires a launcher length in excess of $380 \mathrm{~m}$ to reach it. The critical design parameters of this system reflect materials and launcher technology which is currently available "off the shelf". We see also from the figure that a 
Curve 752331-A

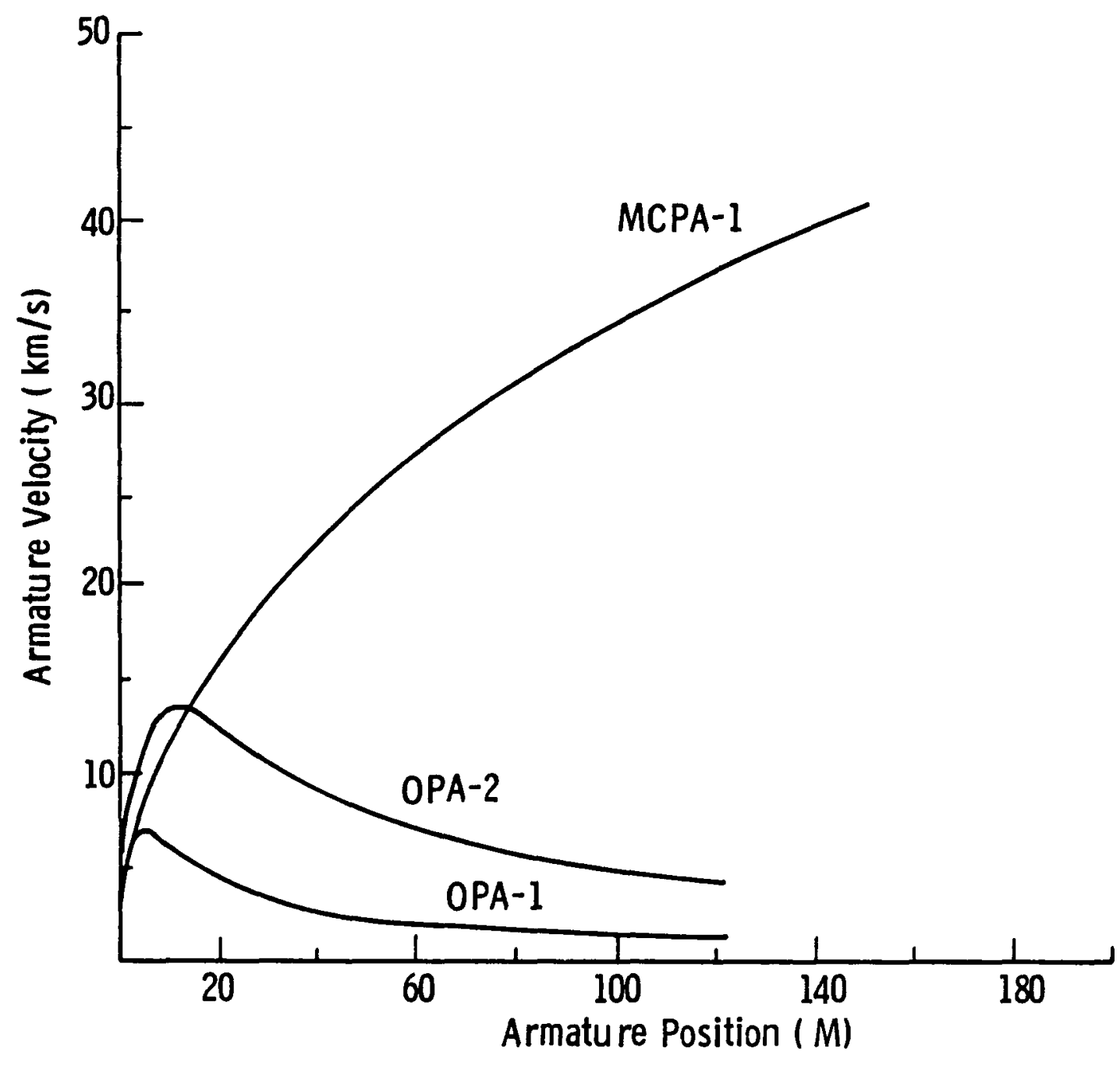

Figure 6.4 - Comparison of theoretical performance of open plasma armature (OPA) and mechanically controlled plasma armature (MCPA) assuming "off-the-shelf" materials, sabot, and railgun barrel technology. 
Curve 752332-A

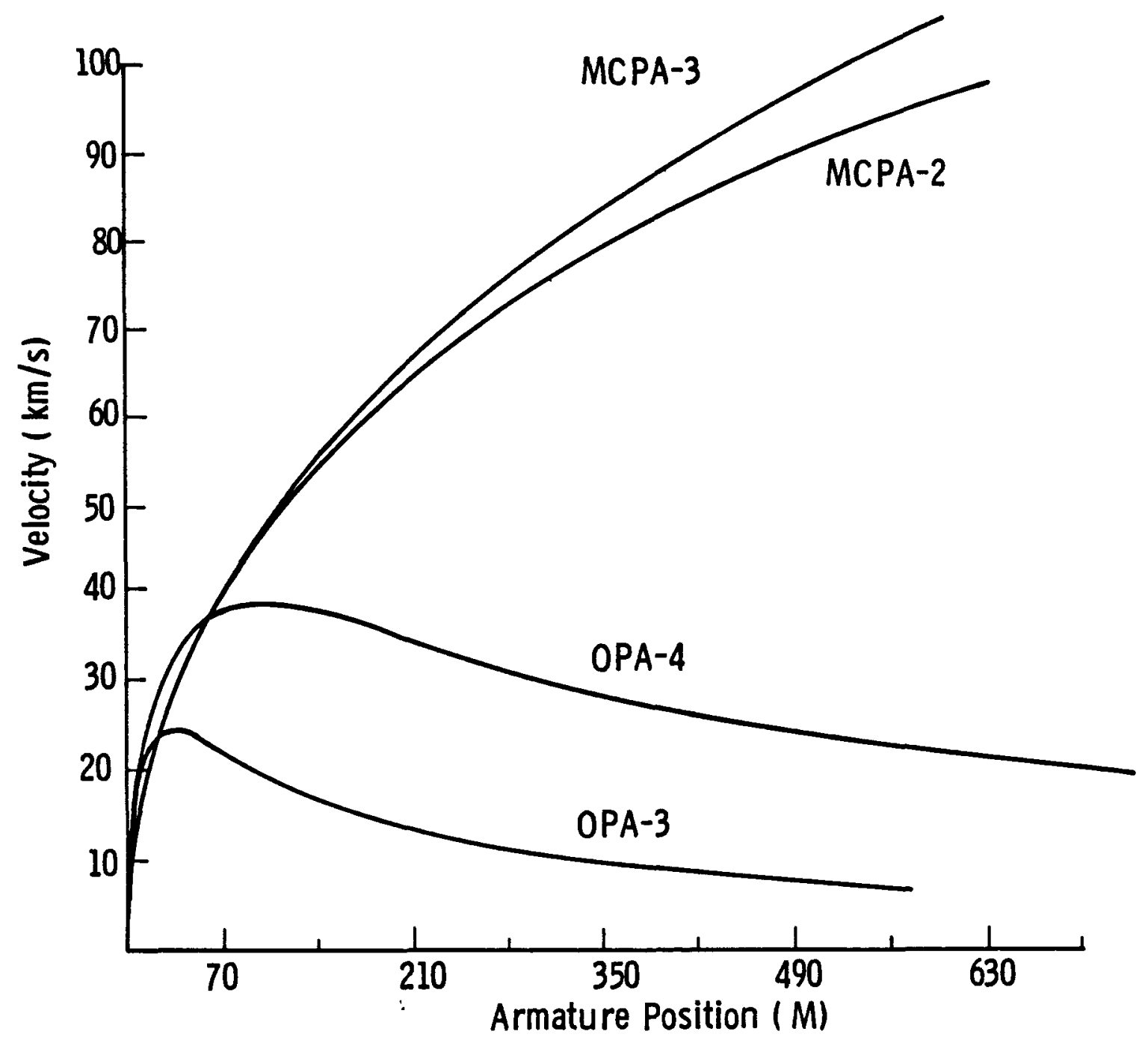

Figure 6.5 - Comparison of theoretical performance of open plasma armature driven railguns and mechanically control led plasma armature driven railguns based upon projected materials, sabot, and railgun barrel technology expected to be developed over the next decade. 
velocity of $15 \mathrm{~km} / \mathrm{s}$ can be achieved with a launcher length of $20 \mathrm{~m}$ despite a relatively massive projectile of $3 \mathrm{~g}$.

In the same figure, Figure 6.4, we have al so shown two other curves which have been labelled as $O P A-1$ and $O P A-2$. The performance predicted by these curves are for systems which use an open plasma armature behind a solid projectile and is calculated from solving the following set of equations governing the launch dynamics in the case of open plasma armature with full or partial estrainment of the ablated mass by the arc, but without the effects of secondary arcs. The formation of secondary arcs would further degrade the performance.

$$
\begin{gathered}
\frac{d \theta}{d t}=\frac{1-\gamma_{1} \theta-\left(1+\gamma_{2} \tau\right) \theta^{2}}{1+\gamma_{1} \tau} \\
\frac{d \zeta}{d \tau}=2 \theta \\
\gamma_{1}=U_{F}\left(\frac{2 \alpha_{b} R_{A}}{L^{\prime}}\right) \\
\gamma_{2}=\frac{M_{T}}{M_{0}} \gamma_{1}
\end{gathered}
$$

The curve OPA-1 shows the performance for the case of a railgun with "standard" design and operating parameters, i.e. an L' of $0.35 \mu \mathrm{H} / \mathrm{m}$, a current per unit rail height of $35 \mathrm{kA} / \mathrm{mm}$, an ablation coefficient of $20 \mathrm{~g} / \mathrm{MJ}$, and an armature resistance of $0.5 \mathrm{~m}$. For a bore of $1 \mathrm{~cm} \mathrm{high}$ and $1 \mathrm{~cm}$ wide, it is possible for the projectile to have a mass as low as $1.2 \mathrm{~g}$. The driving current has been assumed to be as high as $350 \mathrm{kA}$, a relatively high current for this bore size. The skin friction coefficient $c_{f}$ has been assumed to have the same value of 0.001 as before. 
From the figure, we see that, despite the smaller mass of the projectile ( $1 \mathrm{~g}$ versus $3 \mathrm{~g}$ ), and a higher current (350 kA versus $170 \mathrm{kA}$ ) the actual velocity attained by the open armature drive is considerably lower than the velocity attainable by the slotted projectile system. The reason for this is simply due to the use of a high current which leads to a high ablation rate, leading to a rapid accumulation of mass in the arc, consequently giving rise to a high level of viscous drag on the arc. This is in contrast to the case of the slotted projectile system, in which the arc is constantly being 'clipped' by the insulating nozzle, and a constant arc mass is maintained. The maximum velocity for the case of OPA-1 is about $6 \mathrm{~km} / \mathrm{s}$.

We note that in the case of MCPA-1, an ablation rate of $5 \mathrm{~g} / \mathrm{MJ}$ has been assumed, lower than the value $(20 \mathrm{~g} / \mathrm{MJ})$ used in calculating OPA-1. A higher ablation rate in the case of the slotted projectile would actually enhance the launcher dynamics, since it gives rise to a higher mass flow rate for the jet and thus a greater amount of thrust produced by the jet, while the arc mass in the chamber can be maintained at the same value as before by reducing the length of the arc chamber. Consequently the accleration and the final velocity would be higher. However, a higher value for the mass flow rate would put greater demands on the mechanical design of the projectile and the launcher, as the plasma pressure in the chamber would be higher.

The curve OPA-2 is the predicted performance of a system using an open plasma armature, and conforming to the design and operating parameters actually realized in the current SUVAC launcher system, assuming no secondary arc formation. The formation of secondary arcs would severely degrade the launch dynamics. The inductance gradient $L$ ' is $0.7 \mu \mathrm{H} / \mathrm{m}$, the constant driving current is $280 \mathrm{kA}$, and the effective ablation coefficient is $10 \mathrm{~g} / \mathrm{MJ}$. Projectile mass, bore geometry, and skin friction coefficient are as for the case of OPA-1. We see from the figure that, despite these design enhancements, the maximum velocity for 
this open armature drive system is around $12 \mathrm{~km} / \mathrm{s}$, assuming no secondary arcs formation. With the formation of secondary arcs, the maximum velocity attainable is expected to be considerably lower than $12 \mathrm{~km} / \mathrm{s}$.

We also note that, since the magnetic energy density in the bore of the gun for the three systems are nearly the same $(17 \mathrm{~kJ} / \mathrm{m}$ for MCPA-1, $21 \mathrm{~kJ} / \mathrm{m}$ for OPA-1, $27 \mathrm{~kJ} / \mathrm{m}$ for OPA-2), the power supply designed to match the load would have equivalent energy over the same length of the launcher.

The performance indicated by the curves MCPA-1, OPA-1 and OPA-2 are based upon materials, sabot, and launcher technology which are currently available. In Figure 6.5, we show the predicted performance of some systems based upon projected progress in these key technology areas, for both the open-armature drive system and the slottedprojectile drive system. As before, the curves applicable to the slotted-projectile system are labelled with the prefix MCPA, whereas the curves applicable to the systems driven by open plasma armature are labelled with the prefix OPA. The design, operating, and performance parameters are summarised in Table 6.4.

The main features may be described as follows. All systems use an advanced flux augmentation technique (possibly trans-augmentation) to achieve an L' value of $2.5 \mu \mathrm{H} / \mathrm{m}$ and a current of $150 \mathrm{kA}$ to produce a relatively high plasma pressure, but low enough to achieve a low ablation rate. By assuming the use of advanced materials for the rails and the insulators, we assume that ablation rates as low as $5 \mathrm{~g} / \mathrm{MJ}$ (in the case of OPA-3) and $2 \mathrm{~g} / \mathrm{MJ}$ (in the case of OPA-4) can be achieved. Currently, there is a large uncertainty on the precise value of the skin friction coefficient and its dependence on the velocity. For this reason, the cases OPA-4 and MCPA-3 are calculated with a value of 0.0005 for the skin friction coefficient, whereas a value of 0.001 has been assumed for the cases OPA-3 and MCPA-2. Again, for the open-armature 
Table 6.4 - Advanced Launcher and Armature Systems

\begin{tabular}{|c|c|c|c|c|}
\hline & OPA-3 & OPA-4 & MCPA-2 & MCPA-3 \\
\hline Inductance gradient $L^{\prime}(\mu \mathrm{H} / \mathrm{m})$ & 2.5 & 2.5 & 2.5 & 2.5 \\
\hline Current (kA) & 150 & 150 & 150 & 150 \\
\hline Rail separation $(\mathrm{cm})$ & 1 & 1 & 1 & 1 \\
\hline Bore height $(\mathrm{cm})$ & 1 & 1 & 1.2 & 1.2 \\
\hline Jet boost factor & 0 & 0 & 0.2 & 0.2 \\
\hline Armature resistance (m) & 0.5 & 0.5 & - & $\longrightarrow$ \\
\hline Effective ablation $\quad(g / M J)$ & 5 & 2 & - & - \\
\hline Initial arc mass $(\mathrm{mg})$ & 100 & 100 & 20 & 20 \\
\hline Projectile mass $(g)$ & 1.2 & 1.2 & 3 & 3 \\
\hline Skin friction coefficient, $C_{F}$ & $1.0 \times 10^{-}$ & $3.5 \times 10^{-}$ & $1.0 \times 10^{-}$ & $30.5 \times 10$ \\
\hline Maximum velocity $(\mathrm{km} / \mathrm{s})$ & 24 & 40 & 130 & 183 \\
\hline
\end{tabular}

drive systems OPA-3 and OPA-4, a low value for the projectile mass of $1 \mathrm{~g}$ is used, whereas the slotted projectile has a mass of $3 \mathrm{~g}$. Again, since the four systems all produce the same magnetic energy density in the bore of the launcher, matching power supply for driving the four systems would have similar energy level over the same launcher length.

We see that, whereas it may be possible (secondary arcs formation would complicate the results) to use the open-armature drive systems to reach a velocity of $20 \mathrm{~km} / \mathrm{s}$ in the case of OPA-3 and a velocity of $40 \mathrm{~km} / \mathrm{s}$ in the case of OPA-4, the slotted-projectile launcher systems appears to have the potential of reaching velocity in excess of $100 \mathrm{~km} / \mathrm{s}$ for a sufficiently long acceleration length (approx. $500 \mathrm{~m})$. 


\subsection{ADVANCED AUGMENTATION GEOMETRY AND TRANS-AUGMENTATION}

It remains to examine the feasibility of achieving the high degree of flux augmentation as required in the above analysis.

We begin by optimizing the geometrical arrangement of the conductors in the barrel to achieve the maximum degree of coupling between the augmentation condutors and the inner rails, subject to the requirement that these conductors need to withstand the mechanical, thermal, and electrical loading on them and that they have to function together as an integrated barrel structure. One such geometry is shown in Figure 6.6. The conception of this geometry draws upon the basic principle of designing a pulsed air-core transformer. For maximum coupling, the conductors should be 'stacked' on top of each other instead of placing one set of conductors outside the other, as is commonly done in existing augmented railguns. The geometry of

Figure 6.6 was analysed using a finite-element electromagnetic field analysis code with the capability of accurately calculating skin effects and induced eddy currents in a multi-path conductor configuration (WEMAP). With this geometry, a mutual inductance gradient (the highfrequency $(\mathrm{imit})$ as high as $0.31 \mu \mathrm{H} / \mathrm{m}$ is obtained. With the self inductance gradient of the inner rails being $0.57 \mu \mathrm{H} / \mathrm{m}$ (also a relatively high value), the effective inductance gradient of the system is $1.19 \mu \mathrm{H} / \mathrm{m}$ when driven as an augmented railgun in the conventional manner.

To obtain a higher degree of flux augmentation, we could power the augmentation conductors with an independent power source different from the one which powers the inner rails (Figure 6.7). The effective inductance gradient for this system is given by,

$$
L_{\text {eff }}^{\prime}=L_{r}^{\prime}+2\left(I_{a} / I_{r}\right) M^{1}
$$


Dwg. 9383A69

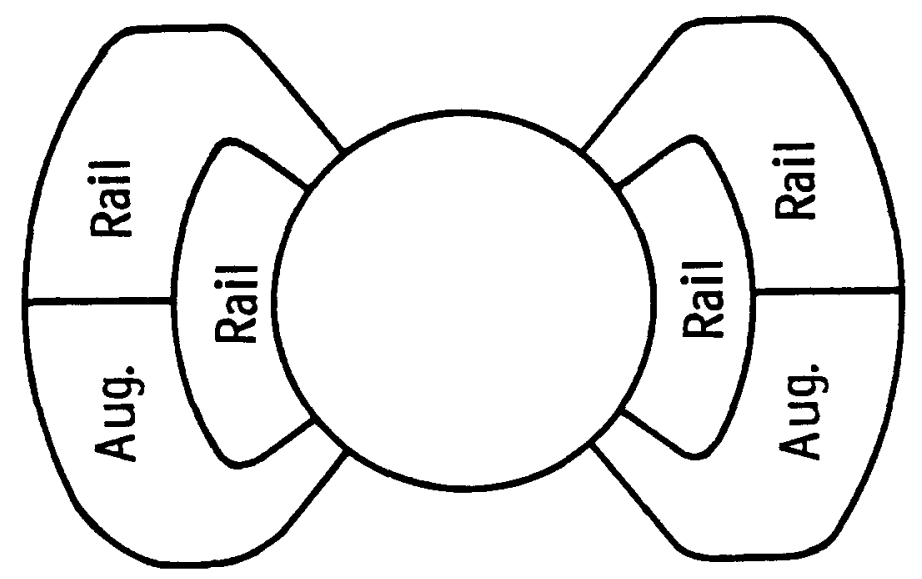

Figure 6.6 - An augmented railgun barrel geometry with close coupling between the augmentation conductors and the inner rails.

Dwg. $9383 A 70$

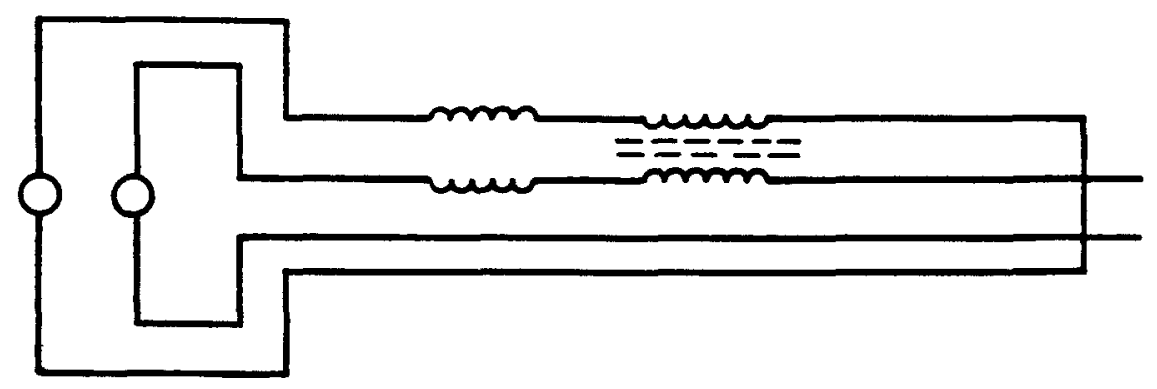

Figure 6.7 - Trans-augmentation: A technique to achieve an extremely high degree of flux augmentation in railguns. 
where $I_{a}$ and $I_{r}$ are the currents in the inner rails and augmentation rails, respectively. ' $L^{\prime} r$ is the self inductance gradient of the inner rails, $M^{\prime}$ is the mutual inductance gradient.

By using a sufficiently large current in the augmentation winding relative to the current in the inner rails, an arbitrarily high value of effective inductance gradient could be obtained. Thus, to achieve an effective inductance gradient of $2.5 \mu \mathrm{H} / \mathrm{m}$ with the barrel geometry of Figure 6.7, an augmentation to inner rails current ratio of 3.2 will be sufficient. 


\section{Reforences}

1. R. S. Hawke, A. L. Brooks, F. J. Deadrick, J. K. Scudder, C. M. Fowler, R. S. Caird and D. R. Peterson, Results of Railgun Experiments Powered by Magnetic Flux Compression Generators", IEEE Trans. Magnetics, MAG-18, 1982, Pp. 82-93.

2. Y. C. Thio, G. A. Clark and A. J. Bedford, "Results from an Experimental Railgun System: ERGS-1A", Report MRL-R-875, Materials Research Laboratories, Defence Science \& Technology Organization, Maribyrnong, Melbourne, Australia, March 1983.

3. A. J. Bedford, G. A. Clark and Y. C. Thio, "Experimental Electromagnetic Launchers at MRL", Report MRL-R-894, Materials Research Laboratories, DSTO, Maribyrnong, Melbourne, Australia, August 1983.

4. G. A. Clark and A. J. Bedford, "Performance Results of a Small-Cal ibre Electromagnetic Launcher", IEEE Trans. Magnetics, MAG-20, March 1984, PP. 276-279.

5. A. J. Bedford, "Rail Damage in a Small Callibre Rail-Gun", IEEE Trans. Magnetics, MAG-20, March 1984, Pp. 245-251.

6. Y. C. Thio, I. R. McNab and W. C. Condit, "Theoretical Performance of Plasma Driven Railguns", Paper AIAA-83-1751, American Institute of Aeronautics and Astronautics, 1983.

7. Y. C. Thio, "Rail Heating by Plasma Armatures in Railguns," Report 84-9J6-AROEM-P1, Westinghouse R\&D Center, 1310 Beulah Road, PA 15235, April 1984.

8. Y. C. Thio and L. S. Frost, Models and Radiation of Railgun Plasma Armatures with Non-Ideal Plasma Properties," Final Report, Contract DAAG29-83-C-0030, US Army Research Office, Research Triangle Park, NC, May, 1986.

9. J. Parker, W. M. Parsons, C. E. Cummings, and W. E. Fox, Merformance Loss due to Wall Ablation in Plasma Armature Railguns," Paper AIAA-85-1575, AIAA 18th Fluid Dynamics, Plasmadynamics and Laser Conference, July 16-18, 1985, Cincinnati, $\mathrm{OH}$. 
10. D. A. Tidman and S. A. Goldstein, "Thermal Transport to Hypervelocity Gun Tubes by High Pressure Partially Ionized Gas Flows," Tech Note GTD-85-4, May, 1985, GT-Devices, 5705A General Washington Drive, Alexandria, VÁ 22312.

11. H. Schlichting, Boundary Layer Theory, 6th Edition, Translated by J. Kestin, McGraw Hill, 1968.

12. B. W. Swanson, Private communication, April, 1986.

13. E. Hantzsche, "Estimation of the Current Density in Cathode Arc Spots", Contrib. Plasma Phys., 25 (5), 1985, pp. 459-465.

14. E. Hantzsche and B. Juttner, "Current density in arc spots", IEEE Trans. Plasma Sci., Vol PS-13, No. 5, 1985, pp. 230-234.

15. S. Anders, B. Juttner, H. Pursch, and P. Siemroth, "Investigations of the Current Density in the Cathode Spot of a Vacuum Arc", Contrib. Plasma Phys., 25 (5), 1985, pp. 467-473.

16. G. Ecker, "Unified Analysis of the Metal Vapour Arc", Z. Naturforsch., 28 a, 1973, pp. 417-428.

17. J. D. Cobine and E. E. Burger, Analysis of electrode phenomena in the high-current arc", J. App. Phys., 26 (7), pp. 895-900, 1955.

18. R. F. Askew, B. A. Chin, B. J. Tatarchuk, J. L. Brown, and D. B. Jensen, "Rail and insulator erosion in rail guns", Proc. 3rd EML Technology Symposium, April 20-24, 1986, pp. 1-5.

19. J. D. Powell, "Thermal Energy Transfer from Arc to Rails in An Arc-Driven Rail Gun", IEEE Trans. Magnetics, Vol. MAG-20, March 1984, pp. 395-398.

20. D. A. Tidman, S. A. Goldstein and N. K. Winsor, "A Rail Gun Plasma Armature Mode," Paper submitted for publications in November 1986 issue of IEEE Transactions on Magnetics.

21. R. A. Marshall, "Plasma puffing from a railgun armature", ibid pp. 264.

22. R. A. Marshall, "Structure of plasma armature of a railgun", Proc. 3rd Symposium on Electromagnetic Launch Technology, April 20-24, 1986, Austin, TX, pp. 165-168. (41)

23. Y. C. Thio, W. C. Condit, T. J. Dougherty, D. L. Ometz, N. A. Ottinger, P. L. Ulerich, D. M. York and J. M. Zomp, SUVAC Annual Report I, 1984. 
24. Y. C. Thio and L. S. Frost, "Non-Ideal Plasma Behavior of Railgun Arcs," Proc. 3rd EM Launcher Technology Symposium, April 20-24, 1986, University of Texas at Austin, Texas. Submitted for publication in November 1986 issue of IEEE Trans. Magnetics.

25. R. E. Kidder, "Nonlinear Diffusion of Strong Magnetic Fields into a Conducting Half-Space," Report Theo. PNU-57, March 1958, Lawrence Livermore National Laboratory, P. 0. Box 808, Livermore, CA 94550.

26. R. S. Hawke and J. K. Scudder, Magnetic Propulsion: Their Design and Capabilities," in Megagauss Physics and Technology, P. J. Turchi, editor, Plenum Press, New York, 1980, pp. 297-312.

27. Y. C. Thio, "PARA: A Computer Simulation Code for Plasma Driven Electromagnetic Launchers", Report MRL-R-873, Materials Research Laboratories, DSTO, Maribyrnong, Melbourne, Victoria, Australia, March 1983.

28. J. D. Powell and J. H. Batteh, "Two-Dimensional Plasma Model for the Arc-Driven Rail Gun", J. Appl. Phys., Vol 54 (5), 1983, pp. 2242-2254.

29. Y. C. Thio, et al. "Investigation of Electromagnetic Launcher Behavior for Impact Fusion" Annual Report for Period May 1, 1984 to April 30, 1985 DOE/ER/13048-2, May 1985.

30. R. A. Marshall and W. F. Weldon, Mnalysis of Performance of Railgun Accelerators Powered by Distributed Energy Stores," 14th Pulse Power Modulator Symposium, Orlando, FL, June 3-5, 1980.

31. J. V. Parker, "Electromagnetic Projectile Accoleration Utilizing Distributed Énergy Sources," J. App. Phys., October 1982.

32. C. H. Haight and M. M. Tower, "Distributed Energy Store (DES) Railgun Development," Proc. 3rd EML Technology Symposium, April 20-24, University of Texas at Austin, TX. Submitted for publication in Nov 1986 issue of IEEE Trans. Magnetics.

33. R. A. Marshall, "Structure of Railgun Plasma Armatures", Proc. 3rd Electromagnetic Launch Technology Symposium, University of Texas, Austin, TX, April 20-24, 1986 . (To be published in IEEE Trans. Magnetics). 